\title{
Electric and magnetic fields inside neurons and their impact upon the cytoskeletal microtubules
}

\author{
Danko Dimchev Georgiev 1, 2 \\ 1 Division Of Electron Microscopy, Medical University Of Varna, Bulgaria \\ 2 Department Of Emergency Medicine, Bregalnitsa Street 3, Varna, Bulgaria
}

If we want to better understand how the microtubules can translate and input the information carried by the electrophysiologic impulses that enter the brain cortex, a detailed investigation of the local electromagnetic field structure is needed. In this paper are assessed the electric and the magnetic field strengths in different neuronal compartments. The calculated results are verified via experimental data comparison. It is shown that the magnetic field is too weak to input information to microtubules and no Hall effect, respectively QHE is realistic. Local magnetic flux density is less than $1 / 300$ of the Earth's magnetic field that's why any magnetic signal will be suffocated by the surrounding noise. In contrast the electric field carries biologically important information and acts upon voltage-gated transmembrane ion channels that control the neuronal action potential. If mind is linked to subneuronal processing of information in the brain microtubules then microtubule interaction with the local electric field, as input source of information is crucial. The intensity of the electric field is estimated to be $10 \mathrm{~V} / \mathrm{m}$ inside the neuronal cytoplasm however the details of the tubulin-electric field interaction are still unknown. A novel hypothesis stressing on the tubulin C-termini intraneuronal function is presented replacing the current flawed models (Tuszynski 2003, Mershin 2003, Hameroff 2003, Porter 2003) presented at the Quantum Mind II Conference held at Tucson, Arizona, 15-19 March 2003, that are shown in this presentation to be biologically and physically inconsistent. 


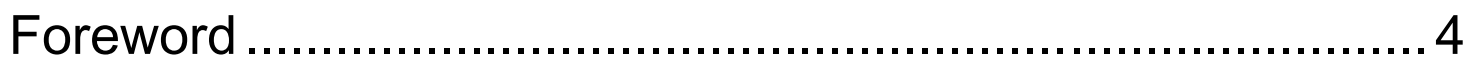

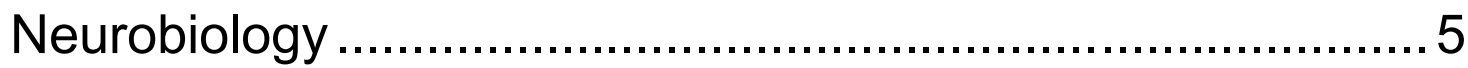

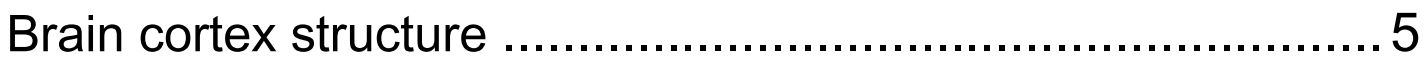

Electromagnetic sensory input to the cortex........................... 6

Neuronal morphology .............................................. 8

The neuronal cytoskeleton ............................................ 12

Electrodynamics ....................................................... 16

Right-handed coordinate systems ...................................... 16

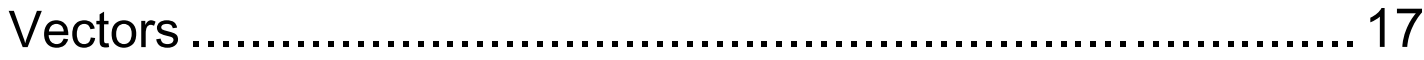

Electric field ......................................................... 18

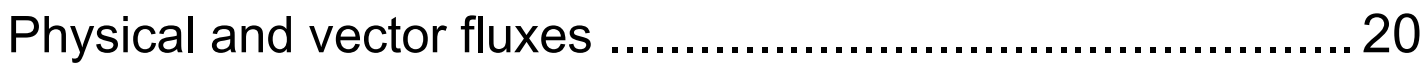

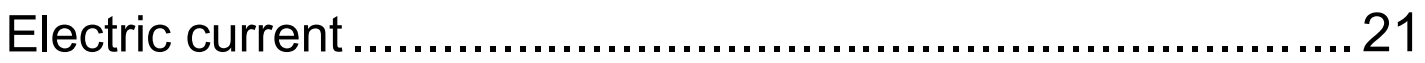

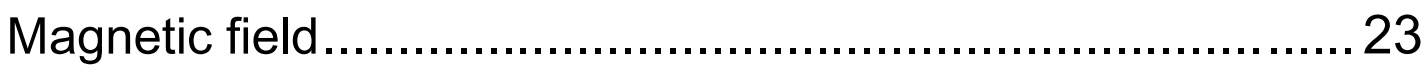

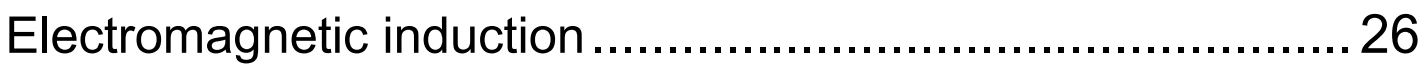

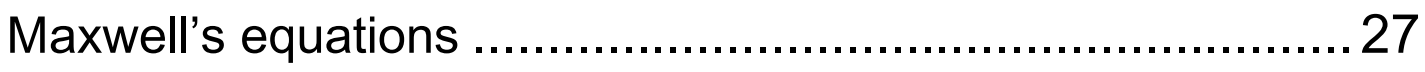

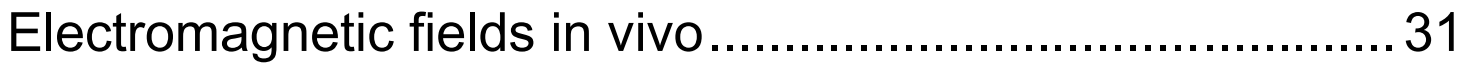

Neuronal membranes as excitable units ............................... 31

Cable equation and dendritic modeling ............................... 33

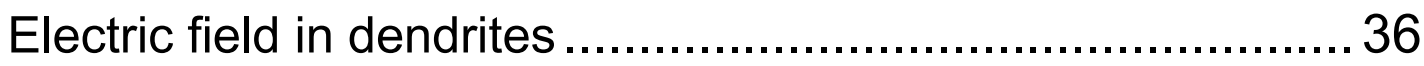

Electric field structure under dendritic spines ...................... 41

Electric currents in dendrites ............................................ 43

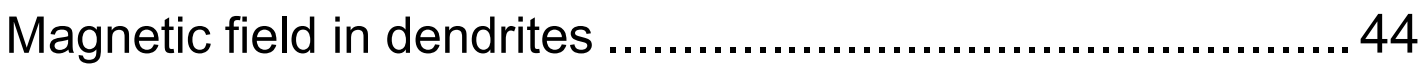

Electromagnetic field in soma ........................................ 45 
Axonal morphophysiology ...................................... 46

The Hodgkin-Huxley model ......................................48

Magnetic field in axons ....................................... 52

Electric field in axons ............................................... 53

Implications for microtubule function .......................... 54

No Hall effect in microtubules............................................54

Microtubule lattice structure ............................................58

Problems in the ferroelectric model of microtubules ..............60

GTP hydrolysis and dynamic instability .............................63

The importance of the water microenvironment ...................65

Tubulin C-termini biological function................................68

Post-translational modification of tubulin tails ......................71

Tubulin tail defects and cerebral pathology .........................73

Processing of information by brain microtubules ..................73

Elastic and piezoelectric properties of microtubules ..............77

Discussion ................................................................... 77

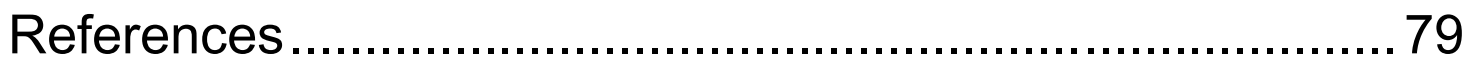




\section{Foreword}

The realization of this project was for the sake of elucidating the mechanisms of inputting the sensory information carried by the membrane potentials that enter the brain cortex and constructing biologically feasible model of subneuronal microtubule based processing of information.

The electric currents are relevant stimuli eliciting conscious experience like memorization of past events (Wilder Penfield, 1954a; 1954b; 1955) and are used to restore the visual image perception in blind man via implanted in the occipital cortex electrodes linked to bionic eye-camera (Dobelle, 2000). The link between the EM field and the cytoskeletal microtubules however was not thoroughly understood. Indeed in the current models presented at the Quantum Mind II Conference, held at Tucson, Arizona, 15-19 March 2003, have been found severe biological and physical inconsistencies. The ferroelectric model (Tuszynski, 2003; Mershin, 2003) leads to extremely high electric field strength needed to polarize microtubules and does not take into account that the suggested $\alpha-\beta$ electron hopping leads to conformational transitions that assemble - disassemble the microtubule. The same error is found in the Orch OR model (Hameroff, 2003a; 2003b) plus additional experimentally disproved predictions about the microtubule lattice, and too slow protein dynamics $(25 \mathrm{~ms})$. The third model predicting topologically stabilized quantum states and anyons suggested by Porter (2003) depends on the magnetic field strength that is responsible for putative quantum Hall effect in the $2 \mathrm{D}$ electron layers presented on the microtubule surface. This idea is not realizable in vivo because of the extremely small magnetic field strength inside neurons assessed to be in the range $10^{-10}-10^{7}$ tesla and because of the millikelvin temperatures needed for QHE. In addition there is little or no data in the presented models how exactly microtubule conformations produce biologically important effects inside neurons. 


\section{Neurobiology}

\section{Brain cortex structure}

The brain cortex is the main residence of consciousness. All sensory stimuli are realized only when they reach the brain cortex and not before that! Nieuwenhuys (1994) outlines the origin and evolutionary development of the neocortex. A cortical formation is lacking in amphibians, but a simple three-layered cortex is present throughout the pallium of reptiles. In mammals, two three-layered cortical structures, i.e. the prepiriform cortex and the hippocampus, are separated from each other by a six-layered neocortex. Still small in marsupials and insectivores, this "new" structure attains amazing dimensions in anthropoids and cetaceans. Neocortical neurons can be allocated to one of two basic categories: pyramidal and nonpyramidal cells. The pyramidal neurons form the principal elements in neocortical circuitry, accounting for at least $70 \%$ of the total neocortical population. The evolutionary development of the pyramidal neurons can be traced from simple, "extraverted" neurons in the amphibian pallium, via pyramidlike neurons in the reptilian cortex to the fully developed neocortical elements designated by Cajal as "psychic cells".

Typical mammalian pyramidal neurons have the following eight features in common: (1) spiny dendrites, (2) a stout radially oriented apical dendrite, forming (3) a terminal bouquet in the most superficial cortical layer, (4) a set of basal dendrites, (5) an axon descending to the subcortical white matter, (6) a number of intracortical axon collaterals, (7) terminals establishing synaptic contacts of the round vesicle/asymmetric variety, and (8) the use of the excitatory aminoacids glutamate and/or aspartate as their neurotransmitter. The pyramidal neurons constitute the sole output and the largest input system of the neocortex. They form the principal targets of the axon collaterals of other pyramidal neurons, as well as of the endings of the main axons of cortico-cortical neurons. 
Indeed, the pyramidal neurons constitute together a continuous network extending over the entire neocortex, justifying the generalization: the neocortex communicates first and foremost within itself!

The neurons from different layers communicate via axo-dendritic synapses, which are chemical informational junctions that transfer information via neuromediator molecules. The neuromediator molecules released from the axonal terminal under depolarization (membrane firing; incoming electric impulse) bind to postsynaptic (dendritic) ion channels, modulate their ion conductivity and generate again electric impulses. Thus the electromagnetic events are essential in neuronal functioning, informational transfer and processing.

\section{Electromagnetic sensory input to the cortex}

The experiments with implanting electrodes directly into the brain cortex suggest that the cortex is the residence for conscious experience. This notion is well supported with clinical data.

William Dobelle, M.D. (2000) has helped a blind man to see again using electrodes implanted into his brain and connected to a tiny television camera mounted on a pair of glasses. Although he does not "see" in the conventional sense, he can make out the outlines of objects, large letters and numbers on a contrasting background, and can use the direct digital input to operate a computer. The man, identified only as Jerry, has been blind since age 36 after a blow to the head. Now 64, he volunteered for the study and got a brain implant in 1978. There has been no infection or rejection in the past 24 years. 
Scientists have been working since 1978 to develop and improve the software that enables Jerry to use the device as a primitive visual system. Jerry's "eye" consists of a tiny television camera and an ultrasonic distance sensor mounted on a pair of eyeglasses. Both devices communicate with a small computer, carried on his hip, which highlights the edges between light and dark areas in the camera image. It then tells an adjacent computer to send appropriate signals to an array of 68 small platinum electrodes on the surface of Jerry's brain, through wires entering his skull behind his right ear. The electrodes stimulate certain brain cells, making Jerry perceive dots of light, which are known as phosphenes. Jerry gets white phosphenes on a black background. With small numbers of phosphenes you have (the equivalent of) a time and temperature sign at a bank. As you get larger and larger numbers of phosphenes, you go up to having a sports stadium scoreboard.

If he is walking down a hall, the doorway appears as a white frame on a dark background. Jerry demonstrated by walking across a room to pull a woolly hat off a wall where it had been taped, took a few steps to a mannequin and correctly put the hat on its head. A reproduction of what Jerry sees showed crosses on a video screen that changed from black to white when the edge of an object passed behind them on the screen. Jerry can read two-inch tall letters at a distance of five feet. And he can use a computer, thanks to some input from his 8-year-old son, Marty. "When an object passes by the television camera ... I see dots of light. Or when I pass by it," Jerry says. The system works by detecting the edges of objects or letters. Jerry, currently the only user of the latest system, must move his head slightly to scan what he is looking at. He has the equivalent of 20/400 vision - about the same as a severely nearsighted person - in a narrow field. Although the relatively small electrode array produces tunnel vision, the patient is also able to navigate in unfamiliar environments including the New York City subway system. 
Another interesting fact is that lesions in the primary visual cortex cause amaurosis corticalis - a condition with decreased visual acuity or even blindness, but with normal pupillar reactions i.e. although there is subcortical neural processing of information, it is not realized because it does not enter the cortex. It can also be concluded that relevant stimulus for the cortical neural cells is the membrane potential, which is then converted into specific quantum states if the Q-mind hypothesis proves true!

\section{Neuronal morphology}

The morphology of a single neuron is relatively simple. In this study is presented a hippocampal pyramidal CA3 (cornu ammonis 3 region) neuron that is typical cortical pyramidal neuron. The neuron has body called soma and two types of projections: dendrites that input electrophysiologic information and axon branching into axonal collaterals that output information via neuromediator release (exocytosis) under membrane firing (depolarization).

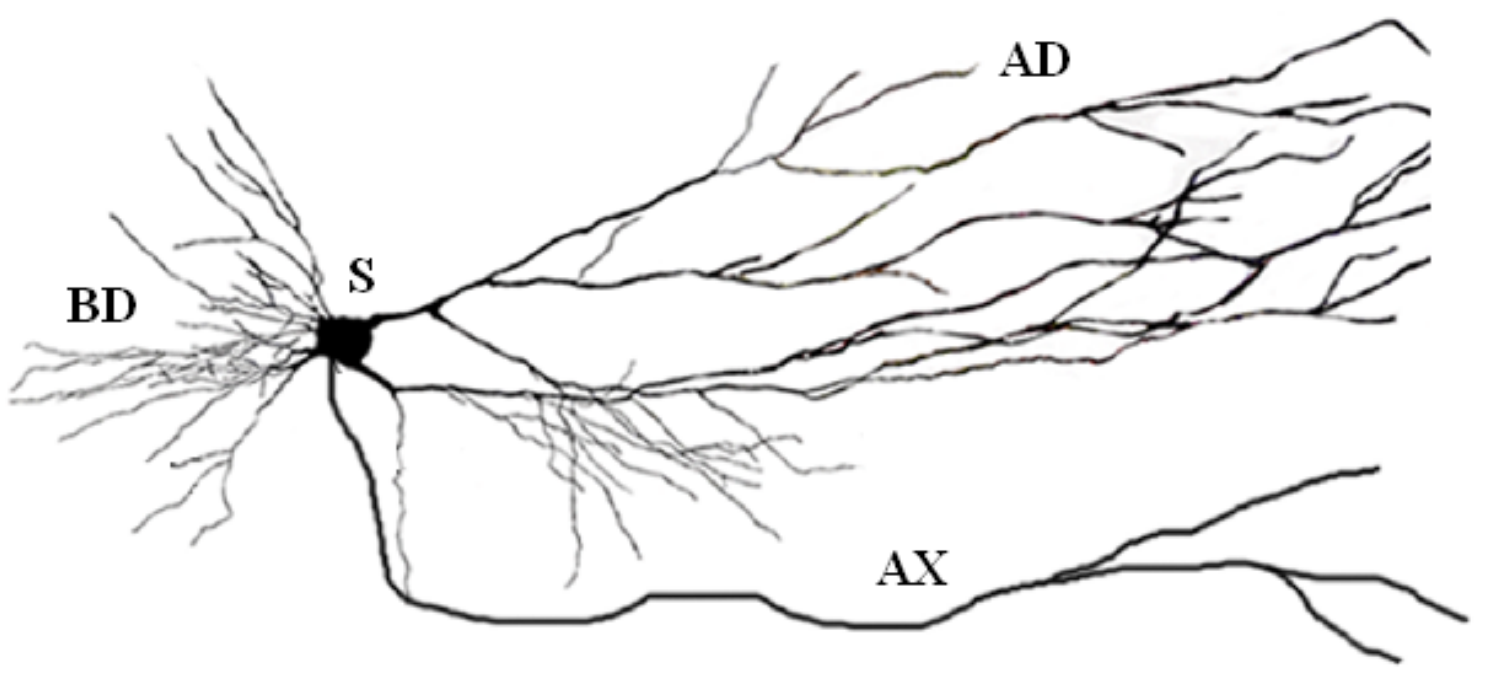

Fig. 1 Structure of hippocampal CA3 neuron. AD, apical dendrites; BD, basal dendrites; S, soma; AX, axon. From Eichler West et al. (1998). 
When cortical pyramidal cells are stained using the immunohistochemical methods we observe that long apical dendrites are extended toward the surface cortical layer and basal dendrites are extended in all directions in the area adjacent to the soma. Dendrites can be thought of as extensions of the cell body with maximal length 1-2 $\mathrm{mm}$ in the largest neurons (Fiala \& Harris, 1999), which provide increased surface area at much lower cell volumes. For example, $97 \%$ of the surface area of a motor neuron (excluding the axon) is dendritic (Ulfhake \& Kellerth, 1981). The dendrites have $3.7 \times 10^{5} \mu \mathrm{m}^{2}$ of surface area while occupying only $3 \times 10^{5} \mu \mathrm{m}^{3}$. To provide an equivalent surface, a spherical cell body would be $340 \mu \mathrm{m}$ in diameter and consume $2 \times 10^{7} \mu \mathrm{m}^{3}$. The fact that $80 \%$ of the surface area of proximal dendrites of motor neurons are covered with synapses (Kellerth et al., 1979) suggests that this increased surface area is indeed valuable for increasing the number of inputs to a neuron.

The most common synaptic specializations of dendrites are simple spines. Spines are protrusions from the dendrite of usually no more than $2 \mu \mathrm{m}$, often ending in a bulbous head attached to the dendrite by a narrow stalk or neck. Spine heads usually have diameter $\sim 0.6 \mu \mathrm{m}$; when this diameter is exceeded we speak about mushroom spines. The spines are usually pedunculated (i.e. they have narrow neck) but sessile spines with no neck are also known. The total spine length for CA1 pyramidal neuron is $0.2-2 \mu \mathrm{m}$, neck diameter $0.04-0.5 \mu \mathrm{m}$, neck length $0.1-2 \mu \mathrm{m}$, total spine volume $0.004-0.6 \mu \mathrm{m}^{3}$, total surface area of a single spine $0.1-4 \mu \mathrm{m}^{2}$, postsynaptic density (PSD) area $0.01-0.5 \mu \mathrm{m}^{2}$.

Neurons are classified as spiny, sparsely spiny, and nonspiny (or smooth) according to the density of simple spines on their dendrites (Peters \& Jones, 1984). Such a classification is complicated by the fact that different dendrites of a given neuron may exhibit widely different spine densities (Feldman \& Peters, 1978). Even along the length of a dendritic segment, spine densities can vary widely. Nominally nonspiny dendrites often exhibit a few simple spines. 
Table I Dendrites in rat CA1 pyramidal cell

\begin{tabular}{|l|l|l|l|l|l|}
\hline $\begin{array}{l}\text { Dendritic } \\
\text { type }\end{array}$ & $\begin{array}{l}\text { Number of } \\
\text { dendrites }\end{array}$ & $\begin{array}{l}\text { Proximal } \\
\text { diameter }\end{array}$ & $\begin{array}{l}\text { Branch } \\
\text { points }\end{array}$ & $\begin{array}{l}\text { Distal } \\
\text { diameter }\end{array}$ & $\begin{array}{l}\text { Dendrite } \\
\text { extend }\end{array}$ \\
\hline $\begin{array}{l}\text { Basal } \\
\text { dendrites }\end{array}$ & 5 & $1 \mu \mathrm{m}$ & 30 & $0.5-1 \mu \mathrm{m}$ & $130 \mu \mathrm{m}$ \\
\hline $\begin{array}{l}\text { Stratum } \\
\text { radiatum }\end{array}$ & 1 & $31 \mu \mathrm{m}$ & 30 & $0.25-1 \mu \mathrm{m}$ & $110 \mu \mathrm{m}$ \\
\hline $\begin{array}{l}\text { Stratum } \\
\text { moleculare }\end{array}$ & & & 15 & $0.25-1 \mu \mathrm{m}$ & $500 \mu \mathrm{m}$ \\
\hline
\end{tabular}

The cytoskeleton of dendrites is composed of microtubules, neurofilaments, and actin filaments. Microtubules are long, thin structures, approximately $25 \mathrm{~nm}$ in diameter, oriented to the longitudinal axis of the dendrite. In regions of the dendrite free of large organelles, they are found in a regular array at a density of 50-150 $\mu^{-2}$. Microtubules are typically spaced 80-200 nm apart, except in places where mitochondria or other organelles squeeze in between them. Microtubules are the "railroad tracks" of the cell and they play an important role in the transport of mitochondria and other organelles (Overly et al., 1996).

The cell body (soma) is the trophic center of the neuron and in CA1 neuron has diameter $\sim 21 \mu \mathrm{m}$. The cell body (soma) contains the nucleus and the principal protein synthetic machinery of the neuron. Axons have essentially no ability to synthesize protein, since they do not contain ribosomes or significant amounts of RNA. Thus, axons depend entirely on proteins produced in the cell body, which are delivered into the axon by important transport processes. Dendrites do contain small amounts of both mRNA and ribosomes, and this protein synthetic machinery is thought to play an important role in dendritic function, but most of the proteins that are present in dendrites are transported from the cell body. 
Because of the large concentrations of ribosomes, neuronal cell bodies stain heavily with basic dyes such as thionin and cresyl violet, which are routine stains for neuropathological studies. In many of the larger neurons, thionin and cresyl violet stains reveal clumps of heavily stained material, termed Niss/ bodies. Nissl bodies represent the stacks of rough endoplasmic reticulum visible at the electron microscopic level.

The distribution of Nissl substance is an indication of the protein synthetic activity of the neuron. When conditions lead to decreases in protein synthesis, there are decreases in Nissl staining. For example, when neurons are axotomized, they often exhibit a response termed chromatolysis, which is characterized by a dispersal of Nissl substance. During the time that the Nissl substance is dispersed, protein synthesis is decreased. Neuronal cell bodies also contain a prominent Golgi apparatus, like other secretory cells. As in other cell types, an important function of the Golgi apparatus of neurons is terminal glycosylation of proteins synthesized in the rough endoplasmic reticulum.

The axon is the output projection that branches into axonal collaterals that reach distant dendrites of target neurons. Study of CA3 -> CA1 axo-dendritic synapses shows that the synapses are formed at $2.7 \mu \mathrm{m}$ intervals along the axons, the axonal shafts are $1.4 \pm 1.2 \mu \mathrm{m}$ long and the varicosities have oblong form and length $-1.1 \pm 0.7 \mu \mathrm{m}$ (Shepherd \& Harris, 1998). It is also possible axo-axonal electric synapses known as gap junctions to account for ultrafast $(200 \mathrm{~Hz})$ signalling between neurons. Axons contain microtubules, usually with closer spacing than in dendrites. 


\section{The neuronal cytoskeleton}

The neuronal cytoskeleton is protein lattice composed from microtubules, intermediary and actin filaments. It was conjectured that the neuronal microtubules are essential subneuronal processors of information i.e. they act as quantum computers that's why in this study we will focus our attention on the interaction between the microtubules and the local electromagnetic field.

Microtubules are hollow cylindrical tubes, of about $25 \mathrm{~nm}$ in diameter on the outside and $14 \mathrm{~nm}$ on the inside, whose walls are polymerized arrays of protein subunits. Their lengths may range from tens of nanometers during early assembly, to possible centimeters in nerve axons within large animals. The protein subunits assemble in longitudinal strings called protofilaments, 13 parallel protofilaments laterally align to form the hollow tubules. The protein subunits are peanut shaped dimers, which in turn consists of two globular proteins, monomers known as $\alpha-$ and $\beta$-tubulin.

The $\alpha$ - and $\beta$-tubulin monomers are similar molecules with identical orientation within protofilaments and tubule walls. In the polymerized state of the MT, one monomer consists of $40 \% \alpha$-helix, 31\% $\beta$-sheet and $29 \%$ random coil. Each monomer consists of about 500 aminoacids, is about $4 \mathrm{~nm} \times 4 \mathrm{~nm} \times 4 \mathrm{~nm}$ and has a local polarity. Each dimer, as well as each MT, appears to have an electric polarity or dipole, with the negative end oriented towards the $\alpha$-monomer and the positive end towards the $\beta$-monomer. The dipole character of the dimer originates from the 18 calcium ions bound within each $\beta$-monomer. An equal number of negative charges required for the electrostatic balance are localized near the neighboring $\alpha$-monomer (Mavromatos et al., 2002). 
The cytoplasmic structural and dynamical organization of cells is due to the presence of networks of interconnected protein polymers, referred to as the cytoskeleton. The cytoskeleton consists of microtubules, actin microfilaments, intermediate filaments and an organizing complex, the centrosome with its chief component the centriole, built from two bundles of microtubules in a separated T-shape. The centrosomal microtubules are unstable and undergo intense dynamics. Much of the dynamic nature of microtubules is attributed to regulated growth and shrinkage of the polymer plus ends (dynamic instability) or to the addition of subunits at the plus end while they are simultaneously lost from the minus end (treadmilling).

The first stage of microtubule formation is called nucleation. The process requires tubulin, $\mathrm{Mg}^{2+}$ and GTP and also proceeds at $37^{\circ} \mathrm{C}$. This stage is relatively slow until the microtubule is initially formed. Nucleation of microtubule assembly can occur spontaneously in a solution of purified tubulin subunits, or it can be stimulated by addition of seeds. Several studies have examined the pathway of early assembly, and conclude that the crucial event in nucleation is formation and growth of a 2-D polymer, the microtubule wall. Investigation by Erickson \& Stoffler (1996) shows that third type of tubulin subunit, namely $\gamma$-tubulin is important for nucleation. $\gamma$-tubulin forms spiral that extends a short length of straight protofilament, which serves as a stable seed for nucleation of a second protofilament. $\alpha / \beta$ subunits form lateral bonds to the $\gamma$-tubulin protofilament, and longitudinal bonds to each other. When this second protofilament has achieved three consecutive $\alpha / \beta$ subunits, growth is more favorable than disassembly and the microtubule should be nucleated. The second phase, called elongation proceeds much more rapidly. The $\alpha-$ and $\beta$-tubulin molecules join to form heterodimers. The heterodimers attach to other dimers to form oligomers, which elongate to form protofilaments. Each dimer carries two GTP molecules. However the GTP that appears to function binds to the $\beta$-tubulin molecules. When a tubulin molecule adds to the microtubule, the 
GTP is hydrolyzed to GDP. Eventually the oligomers will join to form the ringed microtubule. The hydrolysis of GTP of course is facilitated at a temperature of $37^{\circ} \mathrm{C}$ and stopped at temperatures of $4^{\circ} \mathrm{C}$. As the oligomers assemble, they form a series of rings, $25 \mathrm{~nm}$ in diameter.

In the cell itself, microtubules are formed in an area near the nucleus called aster. This is also called the microtubule organizing center (MTOC), or centrosome. Microtubules are polar with a plus end (fast growing) and a minus end (slow growing). Usually the minus end is the anchor point in the MTOC. The plus end carries the GTP molecules, which may be hydrolyzed to GDP. Hydrolysis is not necessary, however. Tests have shown that microtubules will form normally with nonhydrolyzable GTP analog molecules attached, however they will not be able to depolymerize.

It should be stressed that in neurons the microtubule severing by specific enzyme called katanin (Quarmby, 2000) is important for the production of non-centrosomal microtubules, which are stable and thus good candidate for quantum computation. Ahmad et al. (1999) have shown that in neurons a large number of non-centrosomal microtubules is required for the growth and maintenance of neuronal processes.

Injection of an anti-katanin antibody into neurons leads to an accumulation of centrosomal microtubules and a loss of neuronal processes, which indicates that centrosomal katanin, is important for the production of non-centrosomal microtubules primarily through severing of the microtubules near the centrosome. In the neurons the minus end might be capped, which would allow the persistence of centrosome-free microtubules (Rodionov et al., 1999). 
Recent computational study by Baker et al. (2001) has shown that the microtubules have electronegatively charged inner and outer surface and positively charge core. This result suggests existence of two 2-D electron layers (inner and outer) and interesting possibility for anyon formation. Anyons have been found in Nature and could exist in microtubules. They are 2D quasiparticles with partial spin made out of groups of electrons and are ideal candidates for fault-tolerant quantum computation (Porter, 2003; Kitaev, 1997; Mochon, 2003). According to Mitchell Porter (2003) there are four ways in which the microtubule has what it takes to be an anyonic medium: (1) it's two-dimensional - necessary for anyons; (2) it's a cylinder rather than a plane - should turn useless "abelian" anyons into useful "nonabelian" anyons; (3) it's a hexagonal array of qubits - can form domain walls and anyonic defects; (4) the Fibonacci numbers define the geometry of the microtubule. The dependence of the topological anyon model on the magnetic field strength and putative quantum Hall effect (QHE) however is detrimental for its applicability in vivo.

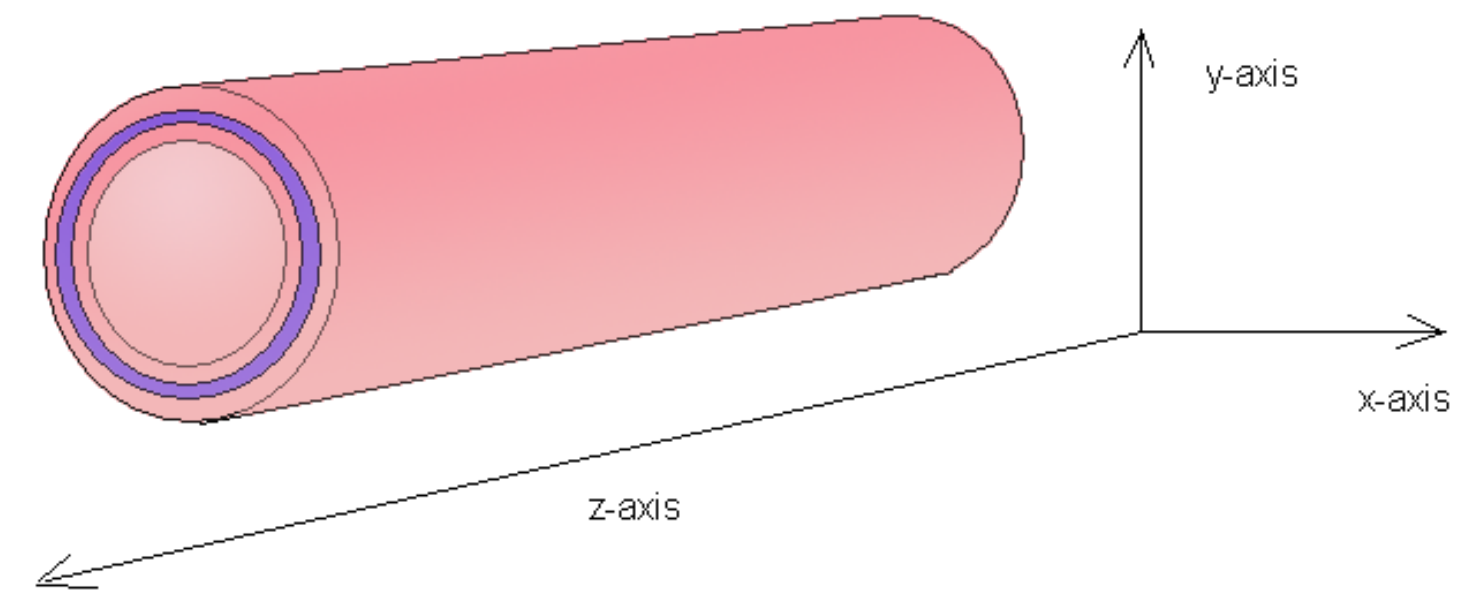

Fig. 2 Microtubule sandwich structure. Cartesian coordinate system attached to the microtubule. The inner and the outer $2 D$ surface of the microtubule are electronegatively charged (red), while the core is electropositive (blue). 


\section{Electrodynamics}

\section{Right-handed coordinate systems}

In order to investigate the electromagnetic field structure it is appropriate to be acquainted with the basic mathematical definitions and physical postulates in classical electrodynamics. First it is worth pointing out that a quantity is either a vector or a scalar. Scalars are quantities, which are fully described by a magnitude alone. Vectors are quantities, which are fully described by both a magnitude and a direction. Because we will work mostly with vectors we have to define what is positive normal to given surface $\mathrm{s}$, what is positive direction of given contour $\Gamma$ and what is right-handed coordinate system?

Right-handed coordinate system Oxyz is such system in which if the z-axis points towards your face the counterclockwise rotation of the Ox axis to the Oy axis has the shortest possible path. The positive normal $+n$ of given surface $s$ closed by contour $\Gamma$ is collinear with the $\mathrm{Oz}$ axis of right-handed coordinate system Oxyz whose $\mathrm{x}$ - and $\mathrm{y}$-axis lie in the plane of the surface. The positive direction of the contour $\Gamma$ is the direction in which the rotation of $x$-axis to the $y$-axis has the shortest possible path.
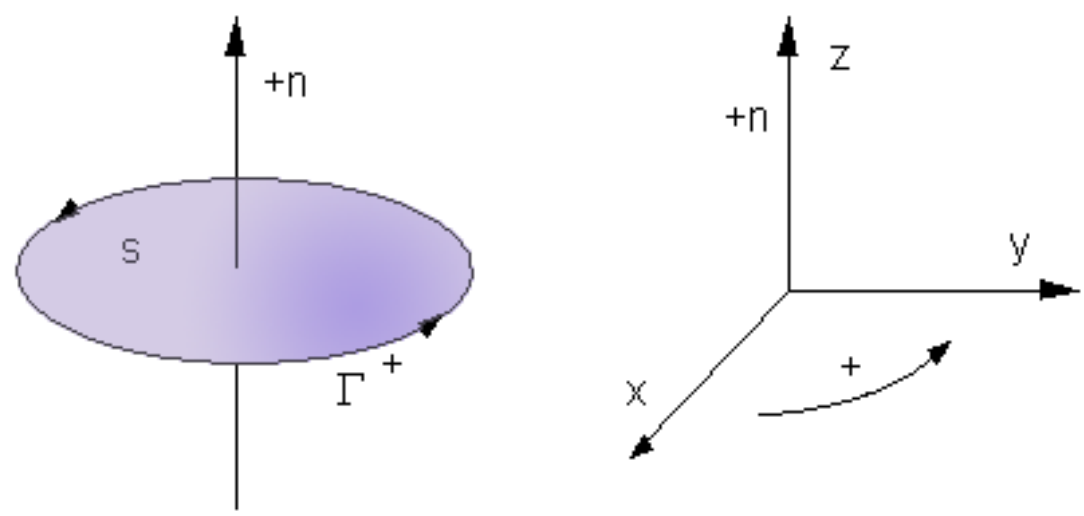

Fig. 3 Direction of the positive normal and right handed coordinate system. 


\section{Vectors}

Because we will work with vectors it should be noted that there are two types of multiplication of vectors - the dot product and the cross product. Geometrically, the dot product of two vectors is the magnitude of one times the projection of the other along the first. The symbol used to represent this operation is a small dot at middle height $(\cdot)$, which is where the name dot product comes from. Since this product has magnitude only, it is also known as the scalar product.

$$
\vec{A} \cdot \vec{B}=A B \cos \beta
$$

where $\beta$ is the angle between the two vectors.

Geometrically, the cross product of two vectors is the area of the parallelogram between them. The symbol used to represent this operation is a large diagonal cross $(x)$, which is where the name cross product comes from. Since this product has magnitude and direction, it is also known as the vector product.

$$
\vec{A} \times \vec{B}=A B \sin \beta \vec{n}
$$

where the vector $\vec{n}$ is a unit vector perpendicular to the plane formed by the two vectors. The direction of $\vec{n}$ is determined by the right hand rule.

The right hand rule says that if you hold your right hand out flat with your fingers pointing in the direction of the first vector and orient your palm so that you can fold your fingers in the direction of the second vector then your thumb will point in the direction of the cross product. 


\section{Electric field}

The electromagnetic field is composed from electric and magnetic field. The electric field could be described via vector field of the electric intensity $\vec{E}$. The electric intensity is defined as the ratio of the electric force $\vec{F}_{E}$ acting upon charged body and the charge q of the body.

$$
\vec{E}=\lim _{\Delta q \rightarrow 0} \frac{\Delta \vec{F}_{E}}{\Delta q}=\frac{d \vec{F}_{E}}{d q}
$$

It should be noted that the electric field is potential field - that is the work W along closed contour $\Gamma$ is zero.

$$
\begin{aligned}
& W_{\Gamma}=\oint_{\Gamma} d W=0 \\
& \oint_{\Gamma} \vec{F}_{E} \cdot d \vec{l}=0
\end{aligned}
$$

where $\mathrm{I}$ is the length of the contour $\Gamma$.

Every point from the electric field has electric potential $V$ defined with the specific (for unit charge) work needed to carry a charge from this point to infinity. The electric potential of point $\mathrm{c}$ of given electric field has potential $\mathrm{V}$ defined by:

$$
V=\int_{c}^{\infty} \vec{E} \cdot d \vec{l}+K
$$

where $V_{\infty}=K=0$. The electric potential difference between two points 1 and 2 defines voltage $\Delta V$ (synonyms: electric potential, electromotive force, potential, potential difference, potential drop). 


$$
\Delta V=\int_{V_{1}}^{V_{2}} d V=\int_{1}^{2} \vec{E} \cdot d \vec{l}
$$

The link between the electric intensity $\vec{E}$ and the gradient of the voltage $\nabla V$ is:

$$
\vec{E}=-\nabla V
$$

where the gradient is a vector operator denoted $\nabla$ called De/ or nabla (Morse \& Feshbach, 1953; Arfken, 1985; Kaplan, 1991; Schey, 1997).

$$
\nabla f \equiv \operatorname{grad}(f)
$$

The gradient vector is pointing towards the higher values of $\mathrm{V}$, with magnitude equal to the rate of change of values. The direction of $\nabla f$ is the orientation in which the directional derivative has the largest value and $|\nabla f|$ is the value of that directional derivative. The directional derivative $\nabla_{u} f\left(x_{0}, y_{0}, z_{0}\right)$ is the rate at which the function $f(x, y, z)$ changes at a point $\left(x_{0}, y_{0}, z_{0}\right)$ in the direction $u$

$$
\nabla_{u} f \equiv \nabla f \frac{u}{|u|}=\lim _{h \rightarrow 0} \frac{f(x+h \vec{u})-f(x)}{h}
$$

and $\vec{u}$ is a unit vector Weisstein (2003).

Another vector not directly measurable that describes the electric field is the vector of the electric induction $\vec{D}$, which for isotropic dielectric is defined as:

$$
\vec{D}=\varepsilon \cdot \vec{E}
$$

where $\varepsilon$ is the electric permittivity of the dielectric. The electric permittivity of the vacuum is denoted with $\varepsilon_{0} \approx 8.84 \times 10^{-12} \mathrm{~F} / \mathrm{m}$. 


\section{Physical and vector fluxes}

The Maxwell's law for the electric flux $\Phi_{D}$ of the vector of the electric induction $\vec{D}$ says that $\Phi_{D}$ through any closed surface $s$ is equal to the exciting the electric field charge $q$ that is located in the space region inside $s$. This could be expressed mathematically:

$$
\begin{aligned}
& \Phi_{D}=q \\
& \oiint_{(s)} \vec{D} \cdot d \vec{s}=q
\end{aligned}
$$

If the normal $+n$ of the surface $s$ and the vector $\vec{D}$ form angle $\beta$ then the flux $\Phi_{D}$ could be defined as:

$$
\begin{aligned}
& \Phi_{D}=\iint_{(s)} \vec{D} \cdot d \vec{s}=\vec{D} \cdot \vec{s}=D \cdot s \cos \beta \\
& d \Phi_{D}=\vec{D} \cdot d \vec{s}=D \cdot d s \cos \beta
\end{aligned}
$$

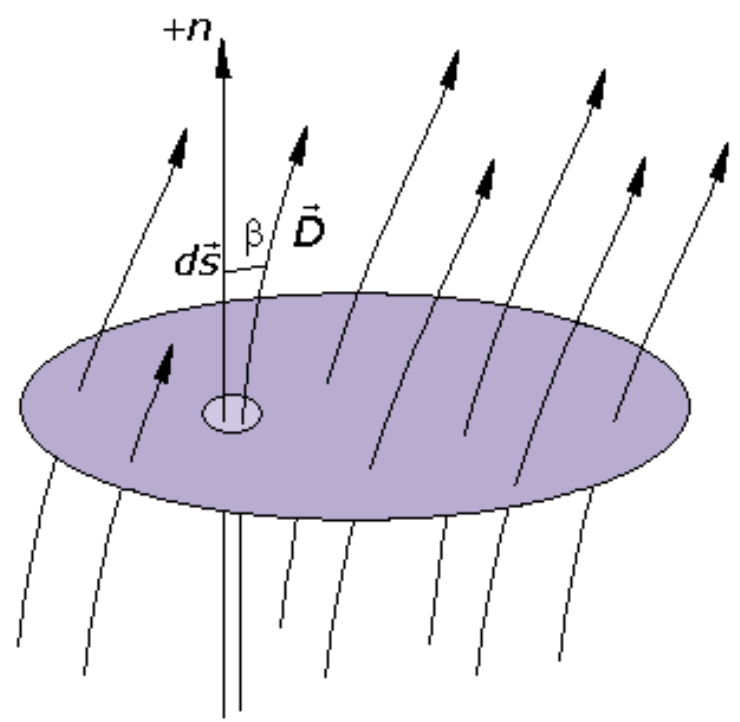

Fig. 4 The flux $\Phi_{D}$ of the vector of the electric induction $\vec{D}$ through surface s. If the surface $s$ is closed then $\Phi_{D}=q$. Modified from Zlatev (1972). 
From the Maxwell's law we could easily derive the Gauss' theorem:

$$
\begin{aligned}
& \Phi_{E}=\frac{q}{\varepsilon} \\
& \oiint_{(s)} \vec{E} \cdot d \vec{s}=\frac{q}{\varepsilon}
\end{aligned}
$$

where $\Phi_{E}$ is the flux of the vector of the electric intensity $\vec{E}$ through the closed surface s. It is important to note that the full electric flux $\Phi_{D}$ could be concentrated only in small region $\Delta s$ of the closed surface $s$, so we could approximate:

$$
\Phi_{D}=\iint_{(\Delta s)} \vec{D} \cdot d \vec{s}=q
$$

In other cases when the electric field is not concentrated in such small region but we are interested to know the partial electric flux $\Delta \Phi_{D}$ through partial surface $\Delta S$ for which is responsible electric charge $\Delta q$ it is appropriate to use the formula:

$$
\Delta \Phi_{D}=\iint_{(\Delta s)} \vec{D} \cdot d \vec{s}=\Delta q
$$

\section{Electric current}

The electric current $i$ that is the flux of (positive) physical charges could be defined using both scalar and vector quantities.

$$
\begin{aligned}
& i=\lim _{\Delta t \rightarrow 0} \frac{\Delta q}{\Delta t}=\frac{d q}{d t} \\
& i=\iint_{(s)} \vec{\jmath} \cdot d \vec{s}=\Phi_{J}
\end{aligned}
$$

where $\vec{j}$ is the density of the electric current. 
As a scalar quantity the current density $\mathrm{J}$ is defined by the following formula:

$$
J=\lim _{\Delta s_{n} \rightarrow 0} \frac{\Delta i}{\Delta s_{n}}=\frac{d i}{d s_{n}}
$$

where $S_{n}$ is the cross section of the current flux $\Phi_{J}$. It is useful to note that usually with $i$ is denoted the flow of positive charges. The flow of negative charges could be easily replaced with positive current with equal magnitude but opposite direction. Sometimes however we would like to underline the nature of the charges in the current and use vectors with indices $\vec{i}_{+}$or $\vec{i}_{-}$where the direction of the vectors coincides with the direction of motion.

If we have cable and current flowing through it, according to the Ohm's law the current $i$ is proportional to the voltage $\mathrm{V}$ and conductance $\mathrm{G}$ and inversely proportional to the resistance $\mathrm{R}$ :

$$
\begin{aligned}
& i=\frac{V}{R}=V \cdot G \\
& R=\rho \frac{I}{S} \\
& G=\gamma \frac{S}{I}
\end{aligned}
$$

where $\rho$ is the specific resistance for the media, $\gamma$ is the specific conductance, $I$ is the length of the cable and $s$ is its cross section. 


\section{Magnetic field}

The magnetic field is the second component of the electromagnetic field and is described by the vector of the magnetic induction $\vec{B}$ (known also as: magnetic field strength or magnetic flux density) that is perpendicular to the vector of the electric intensity $\vec{E}$. The magnetic field acts only on moving charges and manifests itself via electromagnetic force $\vec{F}_{M}$ acting upon flowing currents inside the region where the magnetic field is distributed. From the Laplace's law it is known that the electromagnetic force $\vec{F}_{M}$ acting upon cable with electric current $i \cdot \vec{l}$ that is inside magnetic field with magnetic induction $\vec{B}$ is equal to the vector product of the two vectors:

$$
\begin{aligned}
& \vec{F}_{M}=i \cdot \vec{l} \times \vec{B} \\
& d \vec{F}_{M}=i \cdot d \vec{l} \times \vec{B}
\end{aligned}
$$

If we have magnetic dipole the direction of the vector of the magnetic induction is from the south pole $(\mathrm{S})$ to the north pole $(\mathrm{N})$ inside the dipole and from $\mathrm{N}$ to $\mathrm{S}$ outside the dipole.

The magnetic field could be excited either via changes in existing electric field $\vec{E}$ or by flowing electric current $i$.

In the first case the magnetic induction is defined by the Ampere's law $(\mathrm{J}=0)$ :

$$
\oint_{\Gamma} \vec{B} \cdot d \vec{l}=\varepsilon_{0} \mu_{0} \frac{d \Phi_{E}}{d t}
$$

In the second case if we have cable with current $i$ it will generate magnetic field with induction $\vec{B}$ whose lines have the direction of rotation of right-handed screw when it penetrates in the direction of the current $i$. 

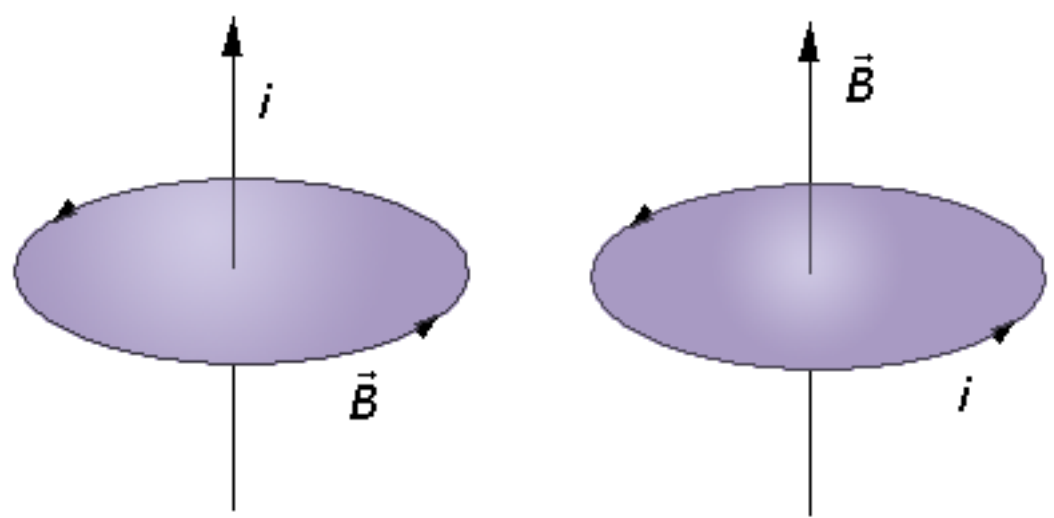

Fig. 5 Direction of the lines of the magnetic induction around axis with current (a) and along the axis of contour with current (b). The current $i$ by convention denotes the flux of positive charges.

The united electromagnetic field manifests itself with resultant electromagnetic force $\vec{F}_{R}$ defined by the Coulomb-Lorentz formula:

$$
\vec{F}_{R}=q\left(\vec{E}+\vec{v}_{i} \times \vec{B}\right)
$$

where $\vec{v}_{i}$ is the velocity of the charge q.

$$
\vec{v}_{i}=\frac{d \vec{l}}{d t}=\frac{d q \cdot d \vec{l}}{d t \cdot d q}=\frac{i \cdot d \vec{l}}{d q}
$$

If we have magnetically isotropic media then we could define another vector describing the magnetic field called magnetic intensity $\vec{H}$ that is:

$$
\vec{H}=\frac{\vec{B}}{\mu}
$$

where $\mu$ is the magnetic permeability of the media. The magnetic permeability of the vacuum is denoted with $\mu_{0} \approx 4 \pi \times 10^{-7} \mathrm{H} / \mathrm{m}$. 
The circulation of the vector of the magnetic intensity $\vec{H}$ along closed contour $\Gamma_{1}$ with length I, which interweaves contour $\Gamma_{2}$ with flowing current $i$ through it, is defined by the formula:

$$
\oint_{\Gamma_{1}} \vec{H} \cdot d \vec{l}=i
$$

It can be seen that the magnetic field is non-potential field since the lines of the field intensity $\vec{H}$ are closed and always interweave the contour with the excitatory current $i$. The circulation of the vector $\vec{H}$ will be zero only along those closed contours that do not interweave the current $i$.

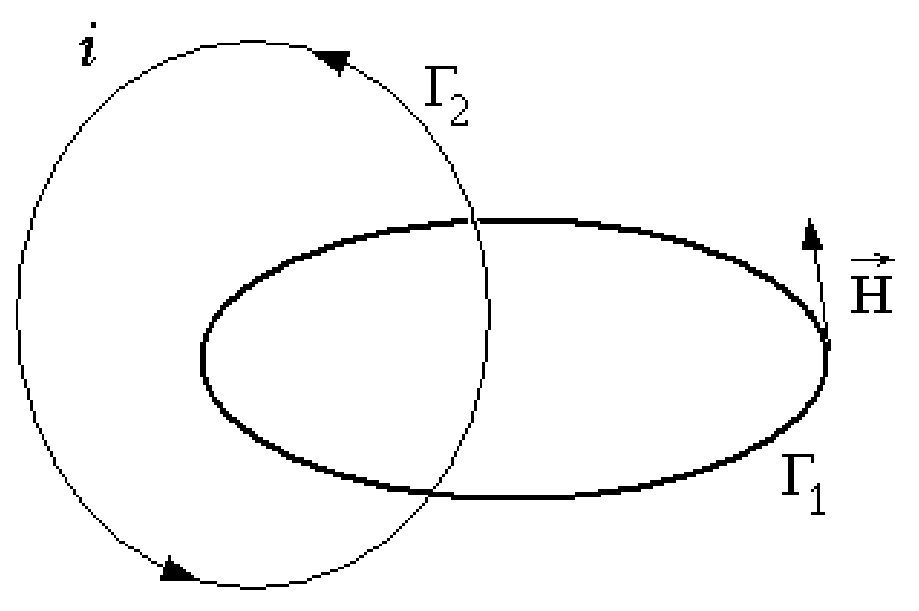

Fig. 6 The circulation of the vector of magnetic intensity $\vec{H}$ along the closed contour $\Gamma_{I}$ equals the current i flowing through the interweaved contour $\Gamma_{2}$. 


\section{Electromagnetic induction}

Analogously to defining the flux $\Phi_{D}$ of the vector of the electric induction $\vec{D}$ we can define the flux $\Phi_{B}$ of the vector of the magnetic induction $\vec{B}$ :

$$
\Phi_{B}=\iint_{(s)} \vec{B} \cdot d \vec{s}
$$

It is useful to know that the change in the magnetic flux generates inducted voltage $V_{\Phi}$ according to the Lenz's law.

$$
\begin{aligned}
& V_{\Phi}=-\frac{d \Phi_{B}}{d t} \\
& \oint_{\Gamma} \vec{E} \cdot d \vec{l}=-\frac{d \Phi_{B}}{d t}
\end{aligned}
$$

Thus the Lenz's law shows that there will be induced current if there is a static cable inside changing magnetic field:

$$
V_{\Phi}=-\iint_{(s)} \frac{\partial \vec{B}}{\partial t} \cdot d \vec{s}
$$

or if the cable is moving inside a static magnetic field:

$$
V_{\Phi}=-[\vec{V} \times \vec{B}] \cdot \vec{l}
$$

The full magnetic flux $\Phi_{L}$ of the self-inducted magnetic field $\vec{B}$ by contour with current $i$ is called self-inducted flux. The self-inducted flux is linear function of the current:

$$
\Phi_{L}=L \cdot i
$$


where $L$ is scalar known as self-inductance and depends only on the magnetic permittivity $\mu$ of the media and the geometric parameters $\Pi_{L}$ that determine the size and the shape of the contour:

$$
L=f\left(\mu, \Pi_{L}\right)
$$

Self-inducted voltage $V_{L}$ appears every time in electric cable when there is change of the current $i$ and this self-inducted voltage opposes to the change of the current:

$$
V_{L}=-L \cdot \frac{d i}{d t}
$$

\section{Maxwell's equations}

In order to summarize the presented basic principles of electromagnetism it is useful to write down the Maxwell's equations. Although these equations have been worked out a century ago they present in concise form the whole electrodynamics.

\section{Integral form in the absence of magnetic or polarizable media}

Gauss' law for electricity

$$
\oiint \vec{E} \cdot d \vec{s}=\frac{q}{\varepsilon_{0}}
$$

Gauss' law for magnetism

$$
\oiint \vec{B} \cdot d \vec{s}=0
$$


Faraday's law of induction

$$
\oint \vec{E} \cdot d \vec{l}=-\frac{d \Phi_{B}}{d t}
$$

Ampere's law

$$
\oint \vec{B} \cdot d \vec{l}=\mu_{0} i+\frac{1}{c^{2}} \frac{\partial}{\partial t} \oint \vec{E} \cdot d \vec{l}
$$

II. Differential form in the absence of magnetic or polarizable media

Gauss' law for electricity

$$
\nabla \cdot E=\frac{\rho}{\varepsilon_{0}}=4 \pi k \rho
$$

where $\rho$ is the charge density and $k=\frac{1}{4 \pi \varepsilon_{0}}$ is the Coulomb's constant.

Gauss' law for magnetism

$$
\nabla \cdot B=0
$$

Faraday's law of induction

$$
\nabla \times E=-\frac{\partial B}{\partial t}
$$

Ampere's law

$$
\nabla \times B=\frac{J}{\varepsilon_{0} c^{2}}+\frac{1}{c^{2}} \frac{\partial E}{\partial t}
$$




\section{Differential form with magnetic and/or polarizable media}

Gauss' law for electricity

$$
\begin{aligned}
& \nabla \cdot D=\rho \\
& D=\varepsilon_{0} E+P
\end{aligned}
$$

where $\mathrm{P}$ denotes the polarization. For free space we have $D=\varepsilon_{0} \cdot E$ and for isotropic linear dielectric $D=\varepsilon \cdot E$. If a material contains polar molecules, they will generally be in random orientations when no electric field is applied. An applied electric field will polarize the material by orienting the dipole moments of polar molecules. This decreases the effective electric field between the plates and will increase the capacitance of the parallel plate structure.

Gauss' law for magnetism

$$
\nabla \cdot B=0
$$

Faraday's law of induction

$$
\nabla \times E=-\frac{\partial B}{\partial t}
$$

Ampere's law

$$
\begin{aligned}
& \nabla \times H=J+\frac{\partial D}{\partial t} \\
& B=\mu_{0}(H+M)
\end{aligned}
$$

where $\mathrm{M}$ denotes the magnetization. For free space we have $B=\mu_{0} \cdot H$ and for isotropic linear magnetic medium $B=\mu \cdot H$. The orbital motion of electrons 
creates tiny atomic current loops, which produce magnetic fields. When an external magnetic field is applied to a material, these current loops will tend to align in such a way as to oppose the applied field. This may be viewed as an atomic version of Lenz's law: induced magnetic fields tend to oppose the change, which created them. Materials in which this effect is the only magnetic response are called diamagnetic. All materials are inherently diamagnetic, but if the atoms have some net magnetic moment as in paramagnetic materials, or if there is long-range ordering of atomic magnetic moments as in ferromagnetic materials, these stronger effects are always dominant (Nave, 2003).

It is interesting to note that the three basic physical constants in electromagnetism: the electric permittivity of vacuum, the magnetic permeability of vacuum and the velocity of light in vacuum are linked by the equation:

$$
\varepsilon_{0} \mu_{0} c_{0}^{2}=1
$$

Taking into account the presented basic laws in the classical electrodynamics we could try to model the electromagnetic field structure and effects taking place in different compartments of the neural cells - dendrites, soma and axons. 


\title{
Electromagnetic fields in vivo
}

\author{
Neuronal membranes as excitable units
}

Physiologically, the electrical signal of relevance to the nervous system is the difference in electrical potential between the interior of a neuron and the surrounding extracellular medium (Schneidman, 2001). The ionic concentration gradients across the cell membrane and the membrane permeability to these ions, determine the membrane potential. The cell membrane is a lipid bilayer, which is impermeable to most ions. Electrically, the membrane is a capacitor separating the charges residing along its inner and outer surface, from both sides. While the resistance of the lipid bilayer by itself is quite high, the resistance of the membrane is significantly reduced by the numerous aqueous pores in the membrane, termed ion channels.

The ions flow into and out of the cell due to both voltage and concentration gradients. However, without external stimuli, these different forces drive the cell to an equilibrium point - the resting membrane potential $V_{m}$ of a neuron, which can be explained from basic physical chemistry principles. Under these resting conditions, the electrical gradient and the ionic concentration gradient balance each other for each of the ion types. The potential inside the cell membrane of a neuron, resulting from the accumulation of charges on the membrane, is then about $-70 \mathrm{mV}$ relative to that of the surrounding bath, and the cell is said to be polarized.

The positive ions (sodium, calcium) that enter the cytoplasm decrease the electronegativity of the membrane potential i.e. they lead to depolarization of the membrane, while the negative ions entering the cytoplasm (chloride) increase the electronegativity of the membrane potential i.e. they hyperpolarize the membrane. The flow of ions from the extracellular space towards the cytoplasm 
is called influx, while the opposite flow is called efflux. Certain ion channels and ion pumps extrude ions from the cytoplasm towards the extracellular space, however here the positive ion efflux (potassium) leads to hyperpolarization, while the negative ion efflux (hydrogencarbonate) leads to depolarization! Normal neuronal membrane potentials vary over a range from about $-90 \mathrm{mV}$ to $+50 \mathrm{mV}$.

The ion channels in the neuronal membranes are ligand or voltage gated. In the first case ligands are the neuromediator molecules that bind to the receptor ion channels and open their gates causing flux of ions. In the second case the ion channels are sensitive to the voltage across the membrane and their gates open and close in concert with the membrane potential changes.

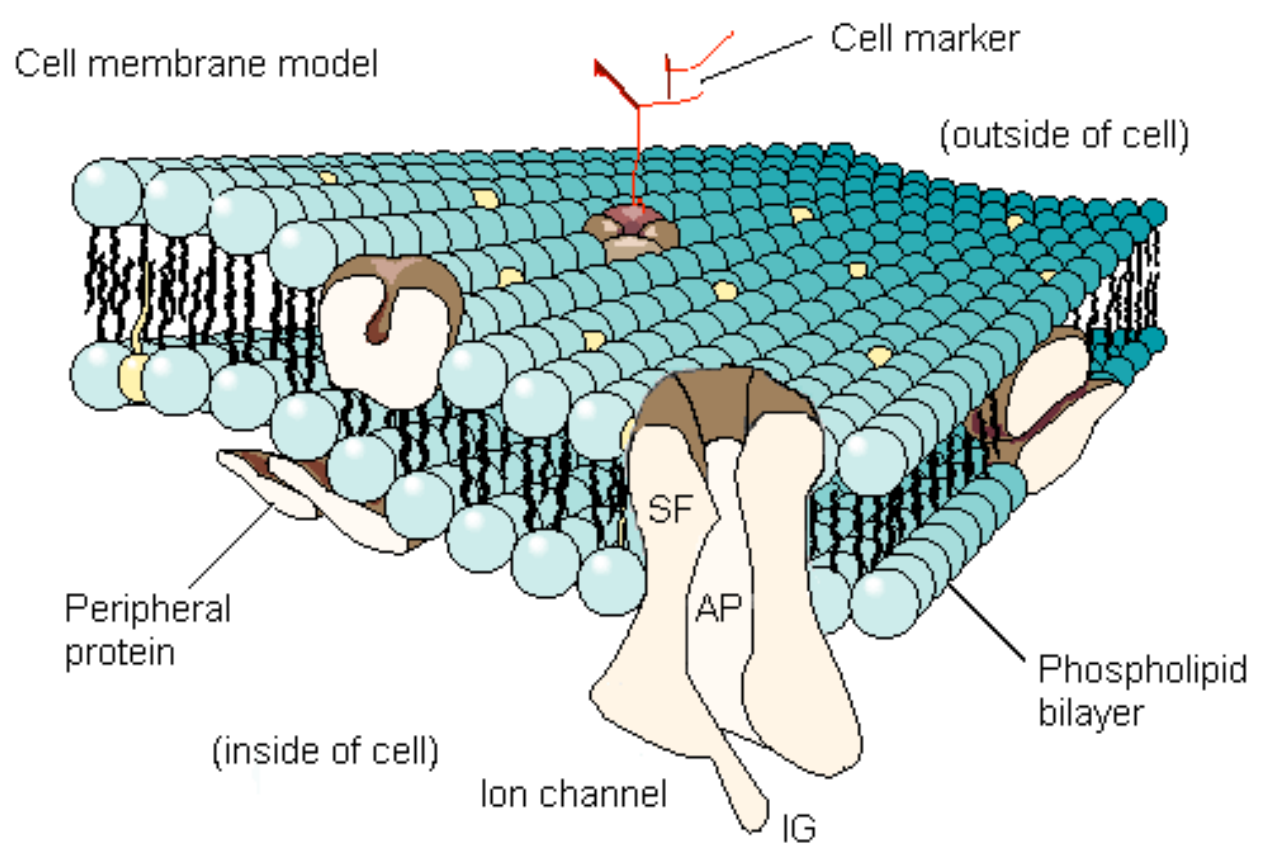

Fig. 7 Membrane lipid bilayer and a single ion channel. Every phospholipid molecule consists of polar head and non-polar tail. The diameter of the ion channel is 3-6 nanometers. Legend: AP, aqueous pore; SF, selectivity filter; IG, inactivation gate. 
Cable equation and dendritic modeling

The main communication between two neurons is achieved via axo-dendritic synapses located at the top of the dendritic spines that are typical for the cortical neurons. It is known that the dendritic postsynaptic membranes convert the neuromediator signal into postsynaptic electric current. The neuromediator molecules bind to specific postsynaptic ion channels and open their gates. The ion species that enter the dendritic cytoplasm change the membrane potential.

The dendritic projections could be modeled as electric cables. That is the electric impulses decrement as they are transmitted by the dendrites. The neuronal membrane could be replaced with equivalent electric schema that takes into account only the passive properties of the membrane.

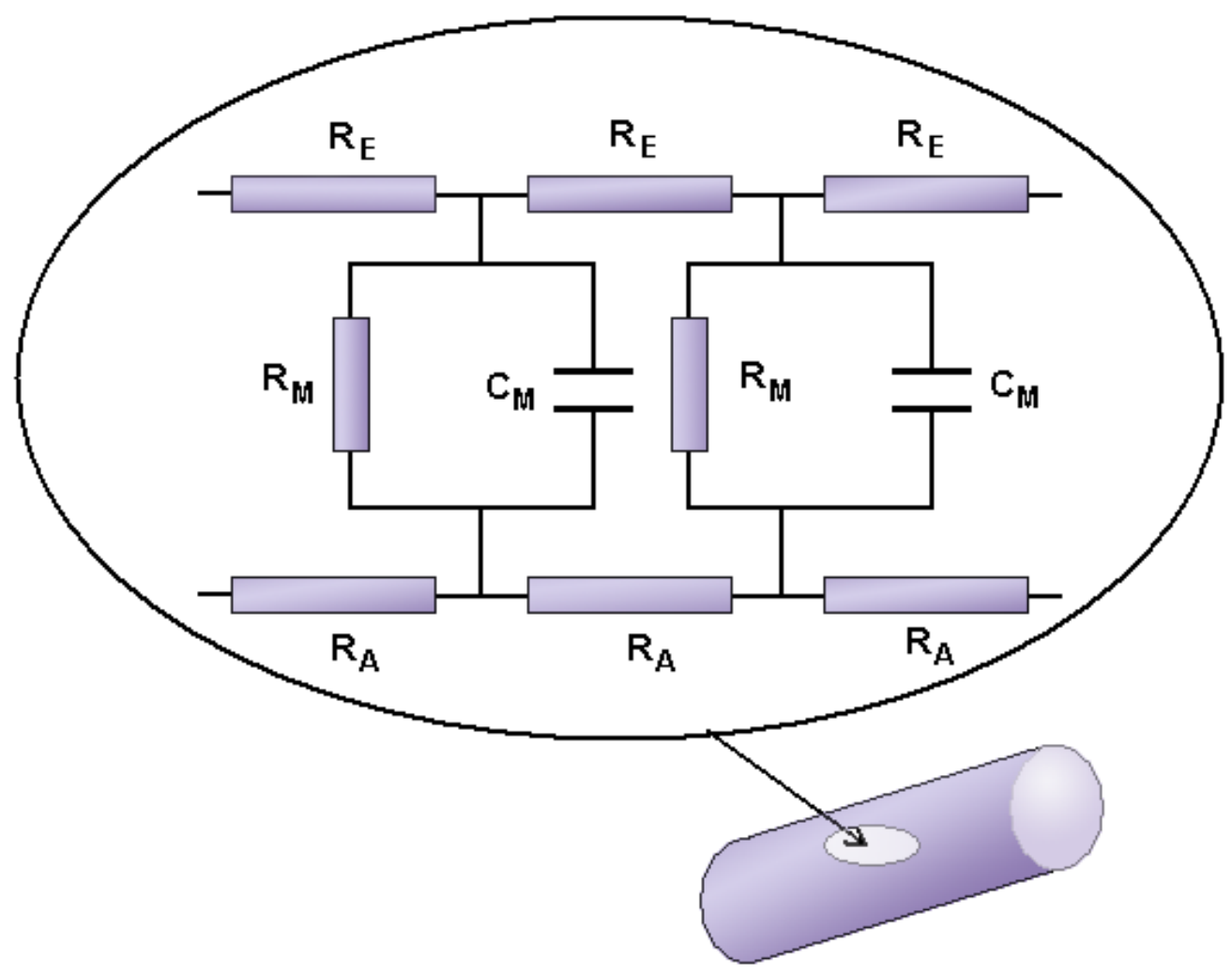

Fig. 8 Equivalent electric schema of passive neuronal membrane. 
Table II Units of the passive membrane. Modified from Paul Sajda (2002).

\begin{tabular}{|c|c|c|c|}
\hline Symbol & SI units & Physical meaning & Notes \\
\hline$R_{a}$ & $\Omega$ & Axial (intracellular) resistance & \multirow{4}{*}{$\begin{array}{l}\text { For a segment of cable } \\
\text { with a fixed length and } \\
\text { fixed diameter }\end{array}$} \\
\hline$R_{e}$ & $\Omega$ & Extracellular resistance & \\
\hline$R_{m}$ & $\Omega$ & Membrane resistance & \\
\hline$C_{m}$ & $\mathrm{~F}$ & Membrane capacitance & \\
\hline$r_{i}$ & $\Omega / \mathrm{m}$ & Cytoplasmic resistivity & \multirow{4}{*}{$\begin{array}{l}\text { For unit length of cable } \\
\text { with fixed diameter }\end{array}$} \\
\hline$r_{e}$ & $\Omega / m$ & Extracellular resistivity & \\
\hline$r_{m}$ & $\Omega . \mathrm{m}$ & Membrane resistivity & \\
\hline$c_{m}$ & $\mathrm{~F} / \mathrm{m}$ & Membrane capacitance & \\
\hline$R_{A}$ & $\Omega . m$ & Specific axial resistance & \multirow{5}{*}{$\begin{array}{l}\text { For unit length and unit } \\
\text { diameter (i.e. unit volume } \\
\text { or surface area of cable) }\end{array}$} \\
\hline$R_{E}$ & $\Omega . \mathrm{m}$ & Specific extracellular resistance & \\
\hline$R_{M}$ & $\Omega . \mathrm{m}^{2}$ & Specific membrane resistance & \\
\hline$G_{M}$ & S. $\mathrm{m}^{-2}$ & Specific membrane conductance & \\
\hline$C_{M}$ & $\mathrm{~F} / \mathrm{m}^{2}$ & Specific membrane capacitance & \\
\hline$V_{m}$ & $\mathrm{~V}$ & Transmembrane voltage & \\
\hline
\end{tabular}

These physical parameters are linked according to the following equations:

$$
\begin{aligned}
& R_{a}=r_{i} I=\frac{4 l}{\pi d^{2}} R_{A} \\
& R_{m}=\frac{r_{m}}{I}=\frac{R_{M}}{\pi d l} \\
& C_{m}=C_{m} I=\pi d I C_{M}
\end{aligned}
$$

where $d$ is the diameter of the neural projection and $I$ is its length. 
Some of the specific parameters were experimentally estimated for real neurons. The specific axial resistance $R_{A}$ is 0.6-1 $\Omega$.m (Miller, 1980; Miller et al., 1985; Fleshman et al., 1988). The value of the specific membrane resistance $R_{M}$ is

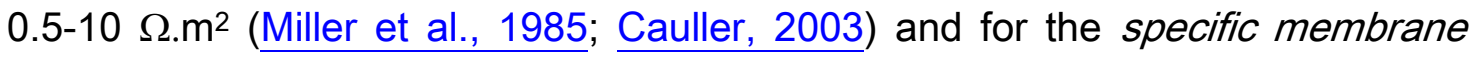
capacitance $C_{M}$ it is $0.01 \mathrm{~F} / \mathrm{m}^{2}$ (Miller et al., 1985).

If we introduce a rectangular electric impulse with voltage $\mathrm{V}$, then the voltage across the membrane changes according to the cable equation:

$$
-\lambda^{2} \frac{\partial^{2} V}{\partial x^{2}}+\tau \frac{\partial V}{\partial t}+V=0,
$$

where

$$
\tau=r_{m} C_{m}=R_{m} C_{m}=R_{M} C_{M}
$$

is the time constant $(\tau)$ and

$$
\lambda^{2}=\frac{r_{m}}{r_{i}+r_{e}}
$$

is the square of the space constant $(\lambda)$.

In neurons $r_{i} \gg r_{e}$ (Sajda, 2002) so we can write:

$$
\lambda=\sqrt{\frac{r_{m}}{r_{i}}}=\sqrt{\frac{d}{4} \frac{R_{M}}{R_{A}}}
$$


The cable equation describes the distribution of the membrane potential in space and time if hyperpolarizing or depolarizing impulse is applied (Stoilov et al., 1985). The time constant $(\tau)$ and the space constant $(\lambda)$ have the meaning respectively of time and distance for which the electric voltage $V$ changes e 2,72 times.

The time constant depends on the membrane resistance that changes in time because the channels do close and open. Dendritic membrane resistivity is shown to be a sigmoidal function (Waldrop \& Glantz, 1985). The time constant depends on the channel conductances (Mayer \& Vyklicky, 1989) and if directly calculated we obtain wide range from $10 \mathrm{~ms}$ to $100 \mathrm{~ms}$.

The space constant depends on the geometry of the neuronal projection and particularly on its diameter. The space constant $\lambda$ for dendrite with $d=1 \mu \mathrm{m}$ is:

$$
\lambda=\sqrt{\frac{d}{4} \frac{R_{M}}{R_{A}}}=\sqrt{\frac{10^{-6} m \times 0.5 \Omega \cdot m^{2}}{4 \times 1 \Omega . m}} \approx 353 \mu \mathrm{m}
$$

Electric field in dendrites

Sayer et al. (1990) have measured the evoked excitatory postsynaptic potentials (EPSP) by single firing of the presynaptic terminal. In their study 71 unitary EPSPs evoked in CA1 pyramidal neurons by activation of single CA3 pyramidal neurons were recorded. The peak amplitudes of these EPSPs ranged from 0.03 to $0.665 \mathrm{mV}$ with a mean of $0.131 \mathrm{mV}$. Recently it become clear that the remote synapses produce higher EPSPs or in other words they 'speak louder' than the proximal synapses so there is no sense to average the EPSPs (Spruston, 2000). In the calculations done further in this paper we will consider that the single EPSP magnitude is $0.2 \mathrm{mV}$ (London \& Segev, 2001). 
In a given point of time the distribution of the voltage along the dendrite is obtained by the cable equation with $V \neq f(t)$ and $\frac{\partial V}{\partial t}=0$ :

$$
-\lambda^{2} \frac{\partial^{2} V}{\partial x^{2}}+V=0
$$

The solution of this differential equation is:

$$
V_{(x)}=V_{0} e^{-x / \lambda}+V_{1} e^{+x / \lambda}
$$

The second part of the equation $V_{1} e^{+x / \lambda}$ could be missed (Stoilov, 1985) because it leads to unphysical results when $x \rightarrow \infty$. Thus we could just write:

$$
V_{(x)}=V_{0} e^{-x / \lambda}
$$

where for $V_{0}$ we will substitute the value of a single evoked EPSP. Here we won't take into account the contribution of another EPSPs that could summate with the EPSP of interest.

If we investigate the change of $\mathrm{V}$ in a single point from the dendrite $(x=0)$ we will see that the impulse decrements with time and the cable equation is reduced to:

$$
\tau \frac{\partial V}{\partial t}+V=0
$$


The solution of this differential equation is:

$$
V(t)=V_{0} e^{-t / \tau}
$$

or the voltage $V$ drops e 2,72 times for time $\tau$ from the end of applied rectangular impulse $\mathrm{V}_{0}$.

In space and time the dynamics of a single EPSP could be described by the following generalized equation:

$$
V_{(x, t)}=V_{0} e^{-x / \lambda} \cdot e^{-t / \tau}
$$

Here should be mentioned that the space constant $\lambda$ depends on the diameter of the dendrite, so we must decompose the dendritic tree into smaller segments with approximately the same $\lambda$ in order to be more precise in our calculations. But if we need rough approximation we could consider that the dendrite has constant diameter of $1 \mu \mathrm{m}$ and we can use the calculated value for the space constant $\lambda \sim 353 \mu \mathrm{m}$.

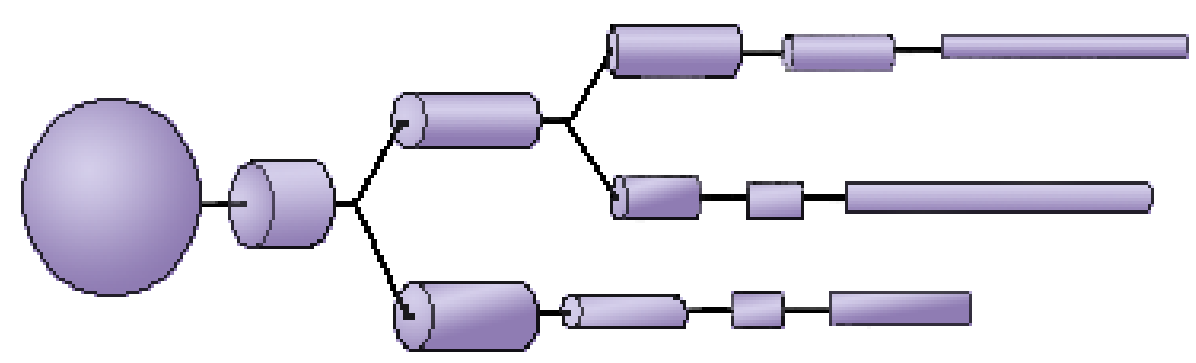

Fig. 9 Cable net approximation of the dendritic tree. Modified from Sajda (2002). 

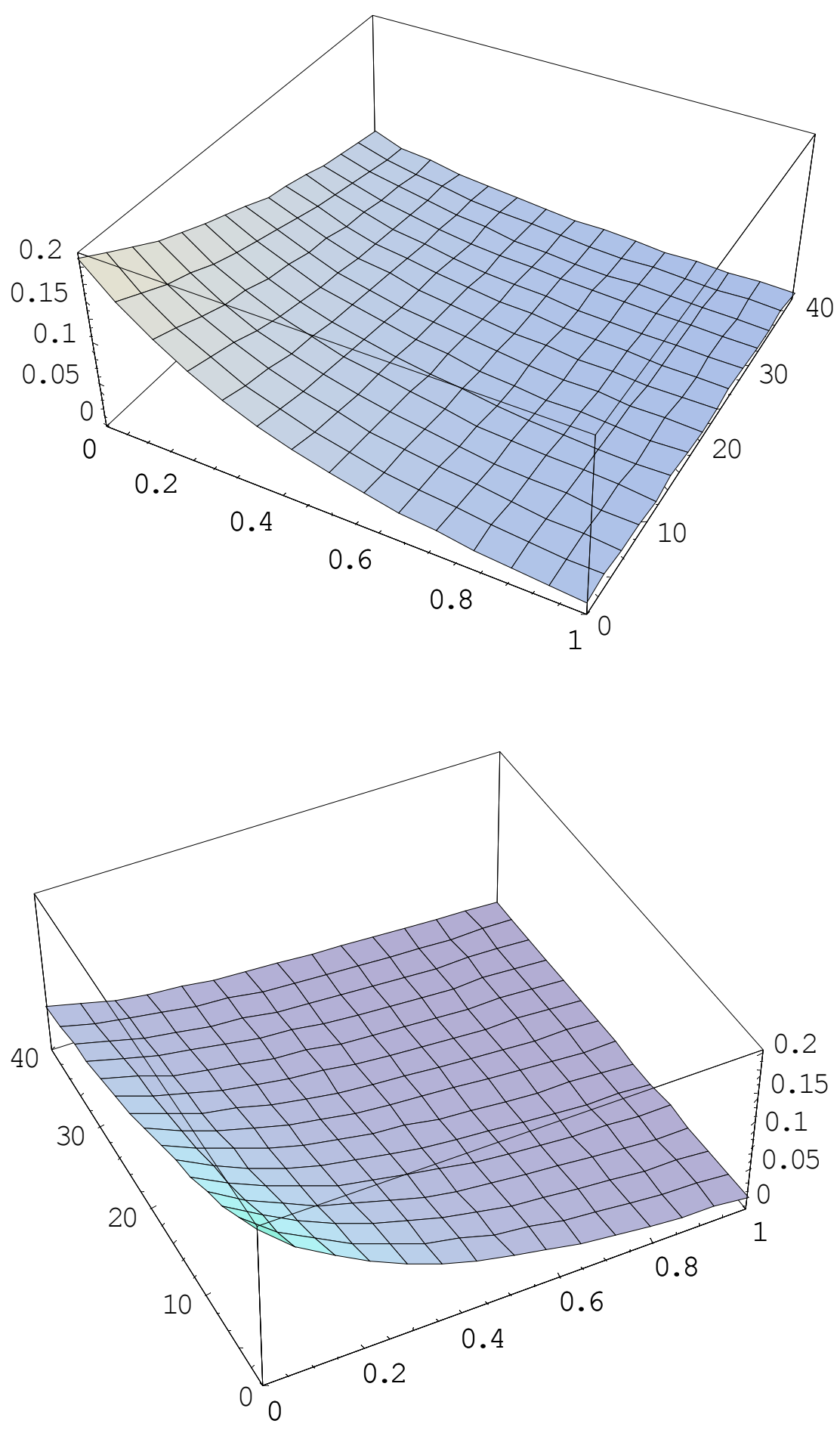

Fig. 10 Spatio-temporal decrement of a single EPSP with magnitude of $0.2 \mathrm{mV}$ in time interval of $40 \mathrm{~ms}$ in dendrite with diameter $d \sim 1 \mu \mathrm{m}$, length $1 \sim 1 \mathrm{~mm}$, space constant $\lambda=353 \mu \mathrm{m}$ and time constant $\tau=30 \mathrm{~ms}$. 
Knowing the distribution of the voltage $V(x, t)$ spread along the dendritic axis we could find the distribution of the electric intensity along the dendrite in space and time after differentiation:

$$
\vec{E}=-\nabla V=-\frac{d V_{(x, t)}}{d x}=\frac{1}{\lambda} V_{0} e^{-x / \lambda} \cdot e^{-t / \tau}
$$

The maximal electric field intensity for a single excitatory postsynaptic potential with magnitude of $0.2 \mathrm{mV}$ is $\sim 0.57 \mathrm{~V} / \mathrm{m}$.

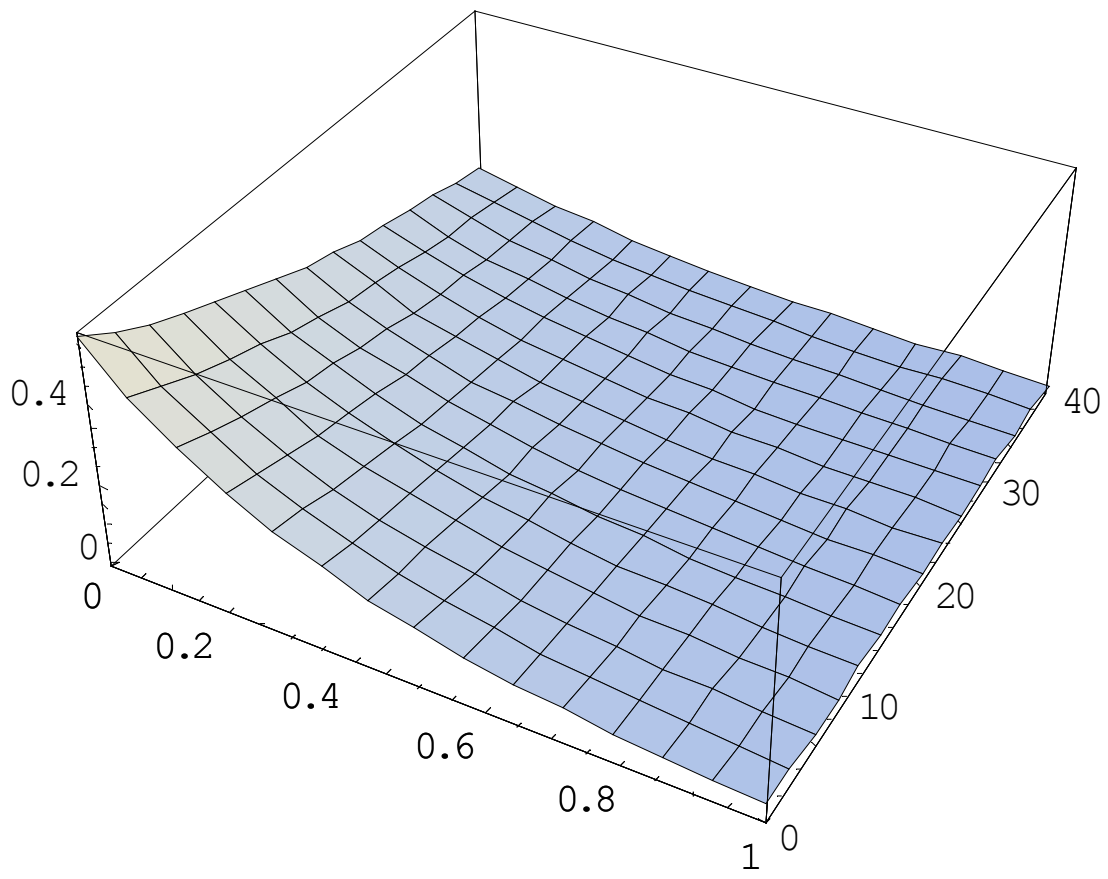

Fig. 11 Distribution of the electric intensity along the axis of the dendrite after application of single EPSP $(0.2 \mathrm{mV})$ at the top of dendrite with diameter $d \sim 1 \mu \mathrm{m}$, length $1 \sim 1 \mathrm{~mm}$ in time interval of $40 \mathrm{~ms}$ ( $\lambda=353 \mu \mathrm{m}$ and $\tau=30 \mathrm{~ms})$. 
Considering that the excitatory postsynaptic potentials (EPSPs) and the inhibitory postsynaptic potentials (IPSPs) could summate over space and time it is not surprise that axial dendritic voltage of tens of millivolts could be measured if there are multiple dendritic inputs. Thus the electric intensity along the dendritic axis in different regions of the dendritic tree could be as high as $10 \mathrm{~V} / \mathrm{m}$ (if $300-400$ EPSPs are temporally and spatially summated). This result is supported by the in vivo estimate of the electric fields ( $E \sim 1-10 \mathrm{~V} / \mathrm{m}$ ) by Jaffe \& Nuccitelli (1977) and the reported data by Tuszynski et al. (1997) that quote intracellular electric intensity values from $0.01 \mathrm{~V} / \mathrm{m}$ to $10 \mathrm{~V} / \mathrm{m}$.

\section{Electric field structure under dendritic spines}

The EPSPs and IPSPs carry information from the presynaptic axonal boutons to the dendritic spines and this information has to be decoded by the underlying microtubules if these subneuronal structures host consciousness.

The dendritic spine consists of spine head (where the synapse is formed) and spine stalk (a narrowing of the spine diameter raising the stalk resistance up to $800 \mathrm{M} \Omega$ (Miller et al., 1985). Based upon the assumption that spine head membrane is passive, previous studies concluded that the efficacy of a synapse onto a spine head would be less than or equal to the efficacy of an identical synapse directly onto the 'parent' dendrite (Chang, 1952; Diamond et al., 1970; Coss \& Globus, 1978). However, for an active spine head membrane, early steady state considerations suggested that spines might act as synaptic amplifiers (Miller et al., 1985). This means that the ion channels located in the spine stalk are voltage-gated and the EPSP propagation will exhibit non-linear properties. When the voltage in the spine stalk reaches definite voltage magnitude the channels open and amplify the synaptic input. 
If we consider that microtubules have acquired mechanisms for translating the information from the electric impulses into specific quantum states then it is demanded to find out a possibility for a microtubule to recognize from which synapse (dendritic spine) the impulse is generated i.e. the geometric structure of the dendritic tree must have specific meaning for microtubules. The vector of electric intensity under the firing dendritic spines is not collinear with the axis of the parent dendrite, therefore $\vec{E}$ is not collinear with the microtubular z-axis in the parent dendrite and angle $\Varangle \xi$ will be formed. Thus the electric field will be carrier of information about specific firing points in the dendritic tree geometry.

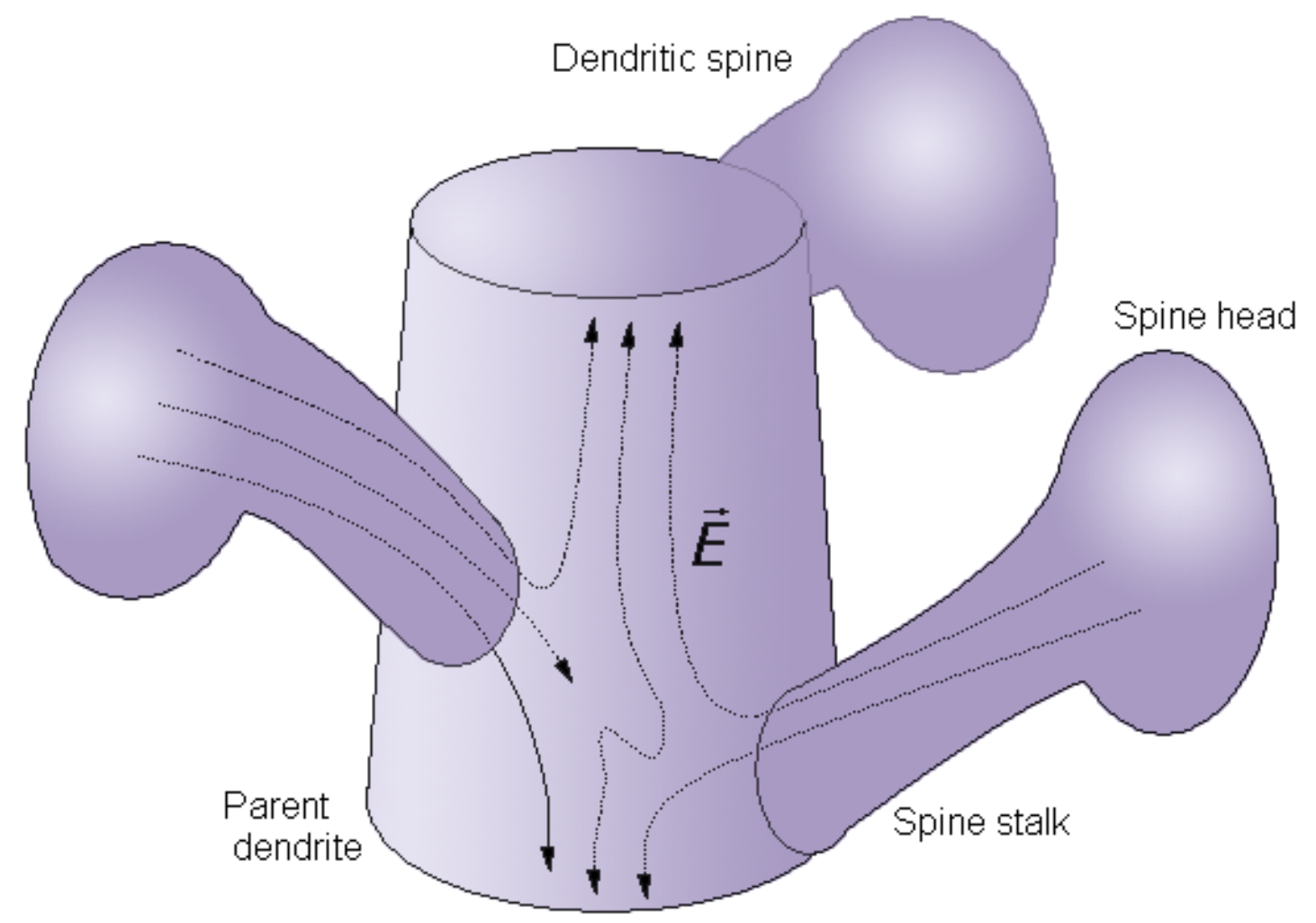

Fig. 12 Distortion of the electric field under the dendritic spines. The $\vec{E}$ vector is not collinear with the $z$-axis of the underlying microtubules under the firing spine stalks. It gets collinear with the z-axis in the areas between two firing stalks including the space under silent dendritic spines. Note: if there is IPSP the $\vec{E}$ vector will point from the parent dendrite towards the spine head. 


\section{Electric currents in dendrites}

From the Ohm's law we could calculate the axial current $i_{a}$ if we know the applied voltage $\mathrm{V}_{0}$ upon the dendritic projection:

$$
i_{a}=\frac{\partial V}{\partial l} \cdot \frac{1}{r_{i}}=\vec{E} \cdot \frac{1}{r_{i}}
$$

where $\mathrm{I}$ is the direction along the axis of the dendrite. The same equation is valid for the axial current outside the dendrite; the only difference is that we should use the $r_{e}$ value. The currents flowing along the dendrite under applied depolarizing or hyperpolarizing impulses are known as local currents. If we have depolarizing impulse there is positive current $\vec{i}_{+}$flowing from the excited area towards the non-excited regions inside the cytoplasm, while outside of the dendrite the positive currents flow towards the place of excitation.

Taking into account that $r_{i}=\frac{4 R_{A}}{\pi d^{2}}$ we obtain:

$$
\vec{i}_{a}=\frac{\partial V}{\partial l} \cdot \frac{\pi d^{2}}{4 R_{A}}=\vec{E} \cdot \frac{\pi d^{2}}{4 R_{A}}
$$

Calculation of the current through the dendrite after applied EPSP with magnitude of $0.2 \mathrm{mV}$ gives us:

$$
\vec{i}_{a}=\frac{0.57 \mathrm{~V} \cdot \mathrm{m}^{-1} \times 3.14 \times 10^{-12} \mathrm{~m}^{2}}{4 \times 1 \Omega m} \approx 0.45 p A
$$

This result is less than the registered evoked inhibitory postsynaptic currents (eIPSCs) which amplitude varies from 20pA to 100pA (Kirischuk et al., 1999; Akaike et al., 2002; Akaike \& Moorhouse, 2003). 
The current density $\mathrm{J}$ through the cross section of the neuronal projection could be calculated from:

$$
\vec{J}=\frac{\vec{i}_{a}}{\vec{s}}=\frac{\partial V}{\partial l} \cdot \frac{1}{R_{A}}=\vec{E} \cdot \frac{1}{R_{A}}=\frac{0.57 V \cdot m^{-1}}{1 \Omega m} \approx 0.57 \mathrm{~A} / \mathrm{m}^{2}
$$

Magnetic field in dendrites

The currents inside dendrites are experimentally measured to be from 20pA to 100pA for GABAergic synapses (Akaike \& Moorhouse, 2003). Using the formula

$$
\oint_{\Gamma} \vec{H} \cdot d \vec{l}=i
$$

we can find the magnetic intensity for a contour $\Gamma$ with length $I=\pi d$ that interweaves the whole current $i_{A}$. For dendrite with $\mathrm{d} \sim 1 \mu \mathrm{m}$ we obtain:

$$
H=\frac{100 \times 10^{-12} A}{3.14 \times 10^{-6} \mathrm{~m}}=31.8 \times 10^{-6} \mathrm{~A} . \mathrm{m}^{-1}
$$

If we consider that the water and the microtubules form a system augmenting the magnetic strength known as ferrofluid (Frick et al., 2003) then in the best-case with effective magnetic permeability $\mu_{\text {eff }} \approx 10$, where $\mu_{\text {eff }}=\frac{\mu}{\mu_{0}}$, we will obtain the maximal magnitude of the magnetic induction $\vec{B}$ inside the neuronal projection:

$$
\begin{aligned}
& \vec{B}=\mu_{\text {eff }} \mu_{0} \vec{H} \\
& B=10 \times 4 \pi \times 10^{-7} H \cdot m^{-1} \times 31.8 \times 10^{-6} A \cdot m^{-1} \approx 4 \times 10^{-10} T
\end{aligned}
$$




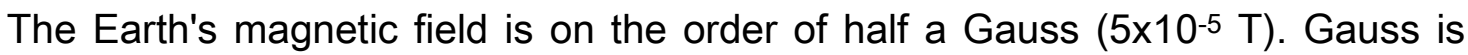
unit used for small fields like the Earth's magnetic field and is $10^{-4} \mathrm{~T}$. It is obvious that the magnetic field generated by the dendritic currents cannot be used as informational signal because the noise resulting from the Earth's magnetic field will suffocate it.

\section{Electromagnetic field in soma}

From computational point of view the neuronal soma integrates the inputs from the dendrites. The passive properties of dendrites lead to decrement of the EPSPs and IPSPs that reach the soma. This however allows for the EPSPs and IPSPs to summate over space and time and when a critical threshold in the soma is reached a non-decrementing axonal spike to occur.

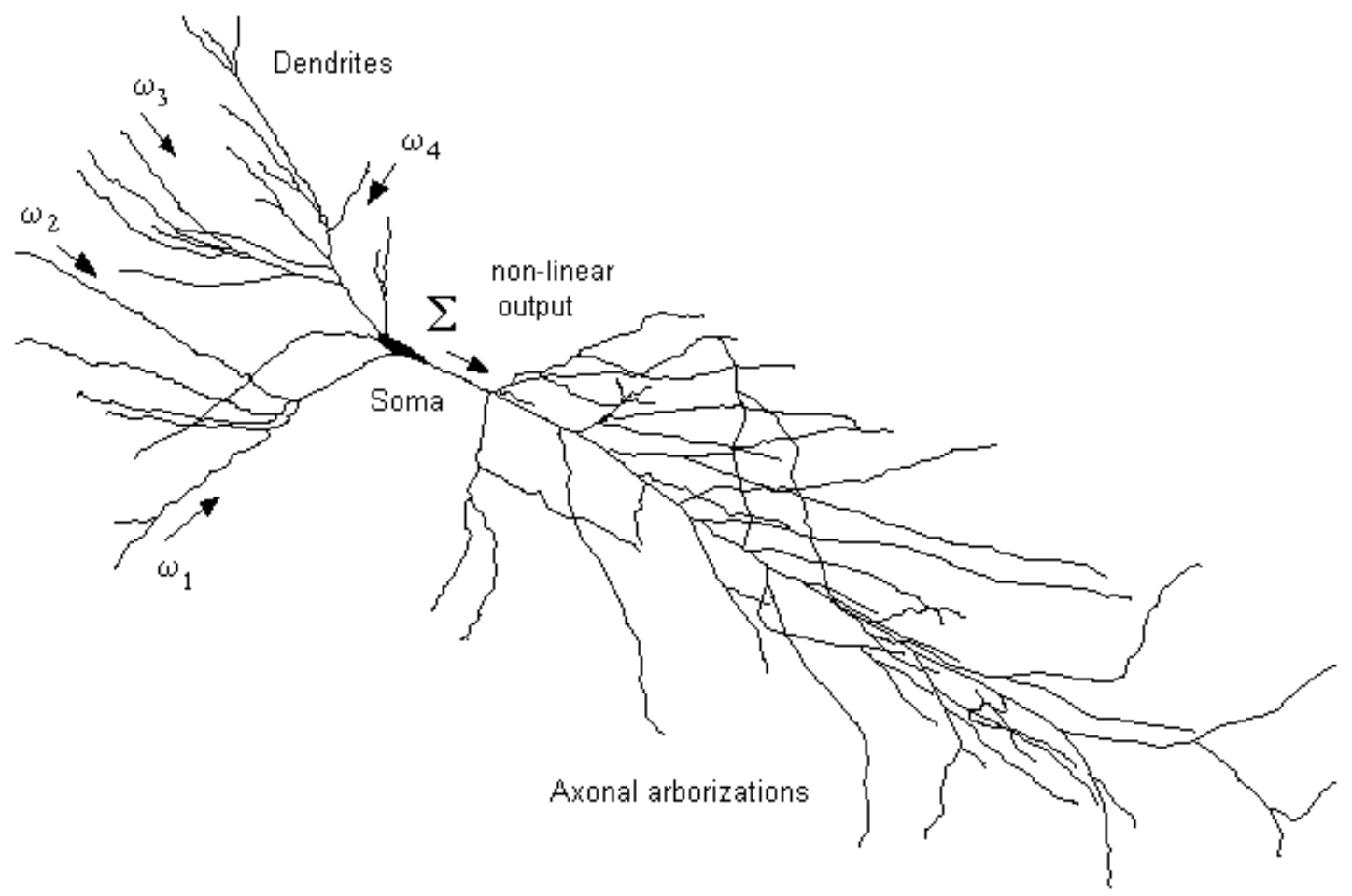

Fig. 13 Standard model of informational processing. Inputs from other neurons are multiplied by the corresponding passive dendritic weights $\omega_{1-}-\omega_{4}$, summed $(\Sigma)$ and then passed through nonlinearity. 
The nonlinear properties of the neuronal output are due to located at the axonal hillock voltage-gated $\mathrm{Na}^{+}$channels that exhibit positive feedback. When a critical threshold of $-55 \mathrm{mV}$ of the transmembrane voltage is reached in the axonal hillock more and more sodium ion channels do open. Considering the decrement along the dendrites a rough estimate of $300-400$ spatially and temporally summated EPSPs are needed in order to elevate the voltage with $15-20 \mathrm{mV}$ in the soma and to evoke axonal spike. The magnetic and the electric field strength are expected to be the same as in the dendrites with maximal magnetic strength of $10^{-10} \mathrm{~T}$ and maximal electric strength of $10 \mathrm{~V} / \mathrm{m}$.

\section{Axonal morphophysiology}

Neurons output information via long projections called axons. The diameter of axons varies from $1 \mu \mathrm{m}$ to $25 \mu \mathrm{m}$ in humans. Axons with small diameter could be non-myelinated. However the larger axons in CNS are ensheathed by multiple membrane layers known as myelin. Myelin is produced by supportive glial cells called oligodendrocytes. Oligodendrocytic membrane rotates around the axon and forms multiple-layered phospholipid structure that insulates the axon from the surrounding environment. One axon is insulated by numerous oligodendrocytes however there are tiny places where the axonal membrane is non-myelinated. They are located between two oligodendrocytic membranes and are called nodes of Ranvier. In the peripheral nervous system the myelin is produced not by oligodendrocytes but by Schwann cells.

When voltage change in the axonal hillock reaches threshold potential of $-55 \mathrm{mV}$, action potential begins. Membrane becomes depolarized due to $\mathrm{Na}^{+}$gates opening, allowing $\mathrm{Na}^{+}$to rush into cell through voltage-gated $\mathrm{Na}^{+}$channels. This is an all-or-none event, in that once threshold is reached, it will happen. When inside of membrane is depolarized to $+40 \mathrm{mV}, \mathrm{Na}^{+}$gates shut and $\mathrm{K}^{+}$gates open. $\mathrm{K}^{+}$rushes out trough open $\mathrm{K}^{+}$gates $\left(\mathrm{Na}^{+}\right.$gates are closed and inactive). 
Membrane becomes re-polarized and may be hyperpolarized (overshoot). Refractory period occurs while $\mathrm{Na}^{+}$gates of the $\mathrm{Na}^{+}$channels remain closed. Membrane will not respond again until $\mathrm{Na}^{+}$gates are active.

The ion currents in axons are greater than the currents in dendrites and the neural impulse known as spike propagates without decrement. This is because the ion channels in the axonal membrane are voltage gated and the current propagation is non-linear. In the myelinated axons the spike jumps from node to node of Ranvier (so called saltatory conduction). The myelin sheath is not permeable for ions (the ion leakage across the membrane is thus prevented) and indeed the sodium and potassium channels are clustered at the Ranvier nodes phenomenon that leads to increase of the conducting velocity!

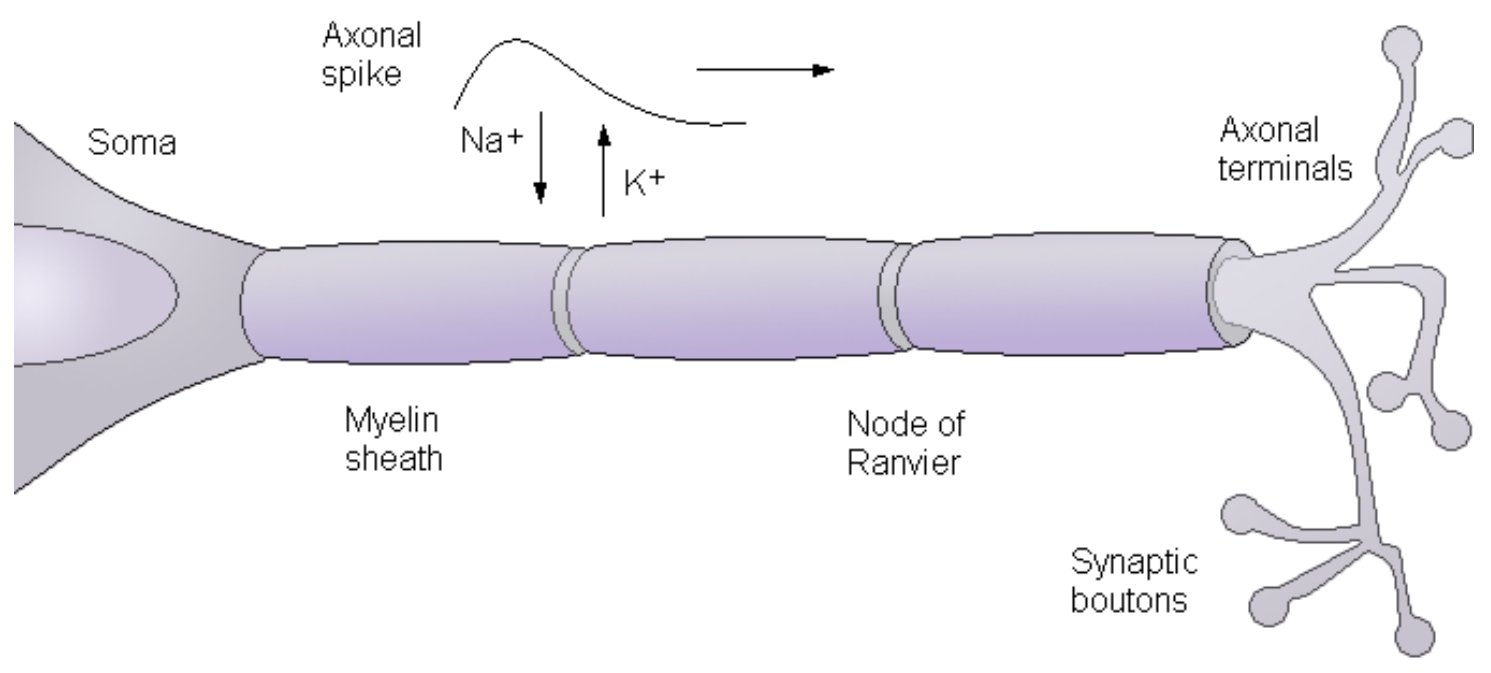

Fig. 14 Axonal spike in myelinated neuron resulting from sodium and potassium ion currents across the membrane in the nodes of Ranvier.

Higher stimulus intensity upon the nerve cell is reflected in increased frequency of impulses, not in higher voltages: all action potentials look essentially the same. The speed of propagation of the action potential for mammalian motor neurons is $10-120 \mathrm{~m} / \mathrm{s}$; while for nonmyelinated sensory neurons it's about $5-25 \mathrm{~m} / \mathrm{s}$ 
(nonmyelinated neurons fire in a continuous fashion, without the jumps; ion leakage allows effectively complete circuits, but slows the rate of propagation).

\section{The Hodgkin-Huxley model}

The current flow across the cell membrane depends on the capacitance of the membrane and the resistance of the ion channels. The total ionic current is represented by the sum of the sodium current, potassium current and a small leakage current. The leakage current represents the collective contribution of ions such as chloride and bicarbonate.

The Hodgkin-Huxley $(\mathrm{HH})$ model is of an isopotential membrane patch or a single electrical compartment i.e. there are no spatial effects on the potential (Hodgkin \& Huxley, 1952a; 1952b; 1952c). The units of the model are per membrane unit area, and it is then straightforward to scale the model to a single compartment of any desired membrane area.

The total membrane current is the sum of the ionic currents and the capacitive current,

$$
\begin{aligned}
& I_{m}(t)=I_{\text {ionic }}(t)+C_{M} \frac{d V_{m}(t)}{d t} \\
& I_{\text {ionic }}(t)=I_{N a}(t)+I_{K}(t)+I_{L}(t)
\end{aligned}
$$

where $I_{m}$ is the membrane current density, $I_{\text {ionic }}$ are the ionic currents densities, $\mathrm{C}_{\mathrm{M}}$ is the membrane capacity per unit area and $\mathrm{V}_{\mathrm{m}}$ is the membrane voltage. The two main ionic conductances, sodium and potassium are independent of each other, and a third, leak conductance does not depend on any of the other conductances or the membranal voltage. Thus, the total ionic current is the sum of the separate ionic currents. 


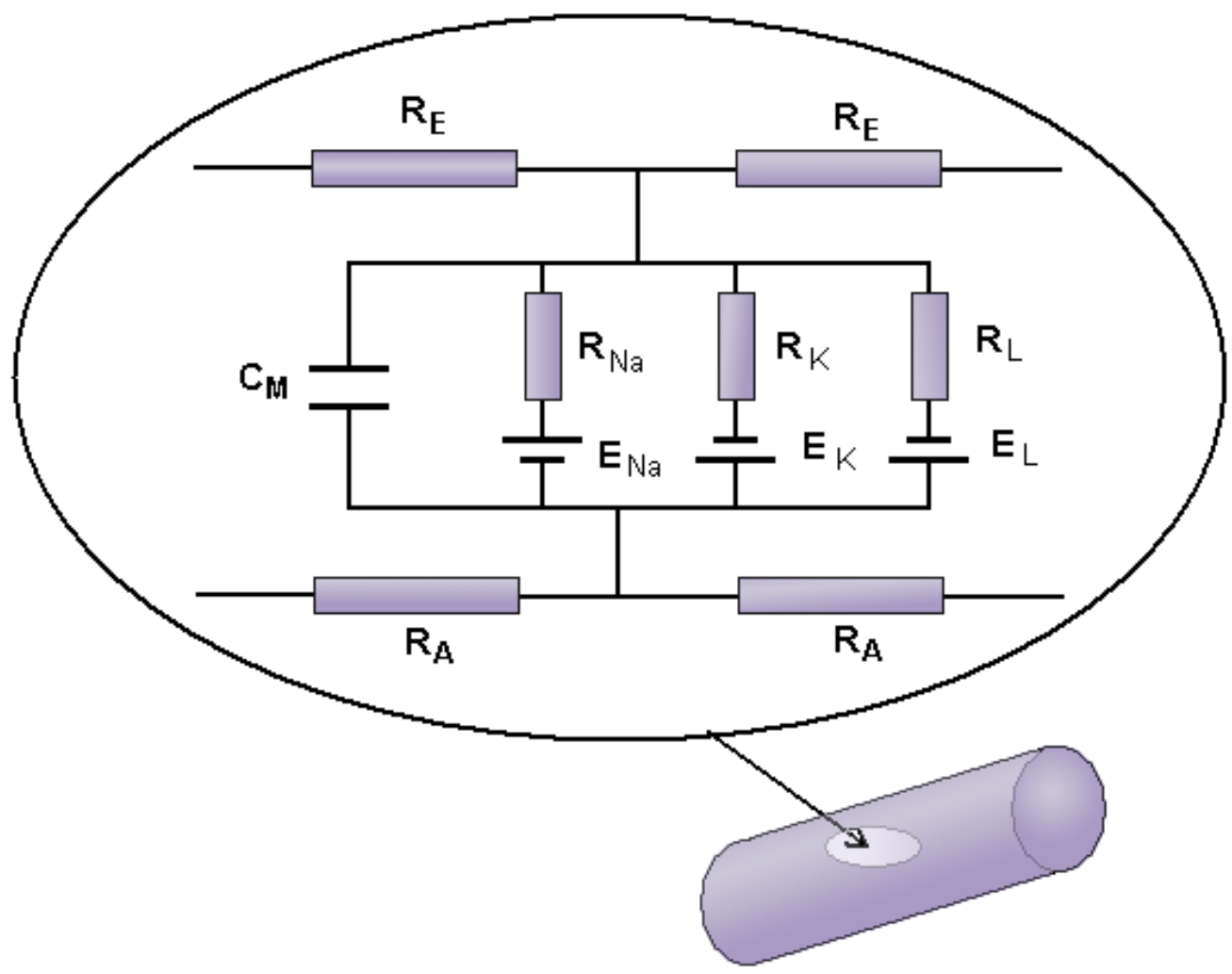

Fig. 15 An electrical circuit diagram describing the current flows across the cell membrane that are captured in the Hodgkin-Huxley model (Schneidman, 2001).

The individual ionic currents are linearly related to the potential according to Ohm's law,

$$
\begin{aligned}
& I_{K}(t)=G_{K}(V, t)\left[V(t)-E_{K}\right] \\
& I_{N a}(t)=G_{N a}(V, t)\left[V(t)-E_{N a}\right] \\
& I_{L}(t)=G_{L}(V, t)\left[V(t)-E_{L}\right]
\end{aligned}
$$

where $G_{K}, G_{N a}$ and $G_{L}$ are the potassium, sodium and leak conductances per unit area of the membrane and $E_{K}, E_{N a}$ and $V_{L}$ are the corresponding reversal or equilibrium potentials of each of the ionic species (the potential at which the ionic concentration gradient is balanced by the electrical potential gradient, and there is no net flux of the ions of this type). 
The equilibrium potential for given ion could be calculated by the Nernst equation:

$$
E_{\text {ion }}=\frac{R T}{F Z} \ln \frac{[\text { Ion }]_{e}}{[\text { Ion }]_{i}}
$$

where $\mathrm{R}$ is the gas constant, $\mathrm{T}$ is the temperature, $\mathrm{F}$ is the Faraday's constant, $\mathrm{Z}$ is the valence of the ion, [lon] and [lon] are the ion concentrations in the extracellular and in the intracellular space.

The voltage-dependent conductances $\mathrm{G}_{\mathrm{Na}}(\mathrm{t})$ and $\mathrm{G}_{\mathrm{K}}(\mathrm{t})$ are given by

$$
\begin{aligned}
& G_{N a}(t)=G_{N a}^{\max } f_{N a}(t) \\
& G_{K}(t)=G_{K}^{\max } f_{K}(t)
\end{aligned}
$$

where $G_{N a}^{\max }$ and $G_{K}^{\max }$ are the peak or maximal sodium and potassium conductances per unit membrane area and $\mathrm{f}_{\mathrm{Na}}(\mathrm{t})$ and $\mathrm{f}_{\mathrm{K}}(\mathrm{t})$ are each the corresponding (instantaneous) fraction of the maximal conductance which is actually open (or active).

Thus the equation, which describes the membrane potential as a function of all the currents that flow across it, is

$$
\begin{gathered}
C_{M} \frac{d V}{d t}=G_{N a}^{\max } f_{N a}(t)\left[E_{N a}-V(t)\right]+G_{K}^{\max } f_{K}(t)\left[E_{K}-V(t)\right] \\
+G_{L}^{\max } f_{L}(t)\left[E_{L}-V(t)\right]+I_{\text {injected }}(t)
\end{gathered}
$$

The values for some of the parameters are: $E_{N a}=+60 \mathrm{mV}, G_{N a}^{\max }=120 \mathrm{mS} / \mathrm{cm}^{2}$, $E_{K}=-93 \mathrm{mV}, G_{K}^{\max }=36 \mathrm{mS} / \mathrm{cm}^{2}, E_{L}=-60 \mathrm{mV}$ and $G_{L}^{\max }=0.3 \mathrm{mS} / \mathrm{cm}^{2}$. 
The $\mathrm{HH}$ model replicates many of the features of spiking of the squid giant axon: the form, duration and amplitude of a single spike (both for the membrane and the propagating spike), its sharp threshold, the conduction velocity of the spike along the axon, the refractory period of the neuron, the impedance changes during the spike, anode-break excitation, accommodation, subthreshold response and oscillations. When simulating the response to sustained stimulus currents, it demonstrates a discontinuous onset of repetitive firing with a high spiking frequency and a limited bandwidth of the firing frequency.

However, careful studies of the model reveal that it does not provide a good description of quite a few electrophysiological properties of the axon (Clay, 1998), in particular the refractory behavior of the preparation in response either to sustained or periodic current pulse stimulation. Also, the model does not account for after potentials and slow changes in the squid giant axon.

Still, the $\mathrm{HH}$ model serves as the 'golden standard' of neuronal excitability, and with minor changes - as the backbone of most neuronal spiking models. The main reason is that the $\mathrm{HH}$ model does capture the essence of spiking through ionic currents $\left(\mathrm{Na}^{+}\right.$and $\left.\mathrm{K}^{+}\right)$, which enter and leave the cell through voltage dependent channels. Moreover, the model is compact, and approximates well many of the features shared by different types of neurons (shape and duration of spiking, repetitive spiking in response to sustained inputs, refractoriness etc.) while incorporating biophysical aspects of the neuron. Adding the appropriate currents for other channel types (usually using similar kinetic schemes) is easily done. Accordingly, and since the model has been studied mathematically in great detail (Jack et al., 1975), it is the common choice of conductance based modeling for computational studies and theoretical ones. 


\section{Magnetic field in axons}

The vector of the magnetic induction $\vec{B}$ will form closed loops around the axis of the neuronal projection and the direction will be defined by the right-handed screw rule (i.e. counterclockwise if the axial current flows toward your face). In axons the magnetic field is stronger than the magnetic field in dendrites because of the greater ion currents flowing inside the axoplasm. The nerve action potential has the form of a moving solitary wave, which can be modeled as two opposing current dipoles driven by a potential change of the order of $70 \mathrm{mV}$. The peak currents range from 5 to $10 \mu \mathrm{A}$ (Katz, 1966). Axons range in diameter from less than $1 \mu \mathrm{m}$ to $25 \mu \mathrm{m}$ in humans, but reach gigantic size in squid $\sim 1 \mathrm{~mm}$. Calculations of the magnetic flux density in the largest human axons that have the greatest electric currents give us:

$$
\begin{aligned}
& B=\mu_{\text {eff }} \mu_{0} \frac{i}{\pi d} \\
& B=\frac{10 \times 4 \times 10^{-7} H \cdot m^{-1} \times 10^{-6} \mathrm{~A}}{25 \times 10^{-6} \mathrm{~m}}=1.6 \times 10^{-7} \mathrm{~T}
\end{aligned}
$$

Although this result is 3 orders of magnitude greater than the experimentally measured magnetic field in frog sciatic nerve using SQUID magnetometer (Wikswo et al., 1980) it remains too weak - only $1 / 300$ of the Earth's magnetic field. The experimentally measured value for the magnetic field using SQUID magnetometer $1.3 \mathrm{~mm}$ aside of the frog sciatic nerve was $1.2 \times 10^{-10} \mathrm{~T}$ with a signal-to-noise ratio 40 to 1 (Wikswo et al., 1980). The assessed value for the magnetic field strength at the nerve surface (where it has peak magnitude) using the Ampere's law

$$
\oint_{\Gamma} B \cdot d l=\mu_{0} i
$$

was $1.2 \times 10^{-10}$ tesla because of large frog sciatic the nerve diameter $(\mathrm{d} \sim 0.6 \mathrm{~mm})$. 


\section{Electric field in axons}

It would be naïve to expect extreme electric field intensities in the axon compared to the field intensity in dendrites. This is because the space constant $\lambda$ in axons is 1-2 orders of magnitude larger than the dendritic space constant and it is inversely linked to the electric field strength $\vec{E}=-\nabla V$. The electric field intensity could be approximated using the cable equation after assessing the space constant $\lambda$ for the axonal projection that increases with the diameter of the neuronal projection:

$$
\lambda=\sqrt{\frac{d}{4} \frac{R_{M}}{R_{A}}}=\sqrt{\frac{25 \times 10^{-6} \mathrm{~m} \times 10 \Omega . \mathrm{m}^{2}}{4 \times 0.62 \Omega . m}} \approx 10 \mathrm{~mm}
$$

The applied voltage could vary from $70 \mathrm{mV}$ to $100 \mathrm{mV}$ (taking into account the overshoot of the action potential) so the electric intensity $\vec{E}$ could reach $10 \mathrm{~V} / \mathrm{m}$.

Neuhaus et al. (2003) note that axonal damage in multiple sclerosis appears to be initiated by increased membrane permeability followed by enhanced $\mathrm{Ca}^{2+}$-influx. Disruption of axonal transport alters the cytoskeleton and leads to axonal swelling, lobulation and, finally, disconnection. The drastic change of the electric microenvironment however could also directly lead to microtubule dysfunction. 


\section{Implications for microtubule function}

\section{No Hall effect in microtubules}

The Hall effect is phenomenon leading to anisotropy of the electroconductivity in a conductor or semi-conductor and is caused by magnetic field whose induction $\vec{B}$ is perpendicular to the vector describing the current density $\vec{\jmath}$. In this anisotropy the vector $\vec{j}$ and the electric intensity vector $\vec{E}$ are not collinear, but form angle $\theta_{H}$ between them, called Hall angle. Hall effect, discovered in 1879, is almost invisible in metals but is much more pronounced in semi-conductors!

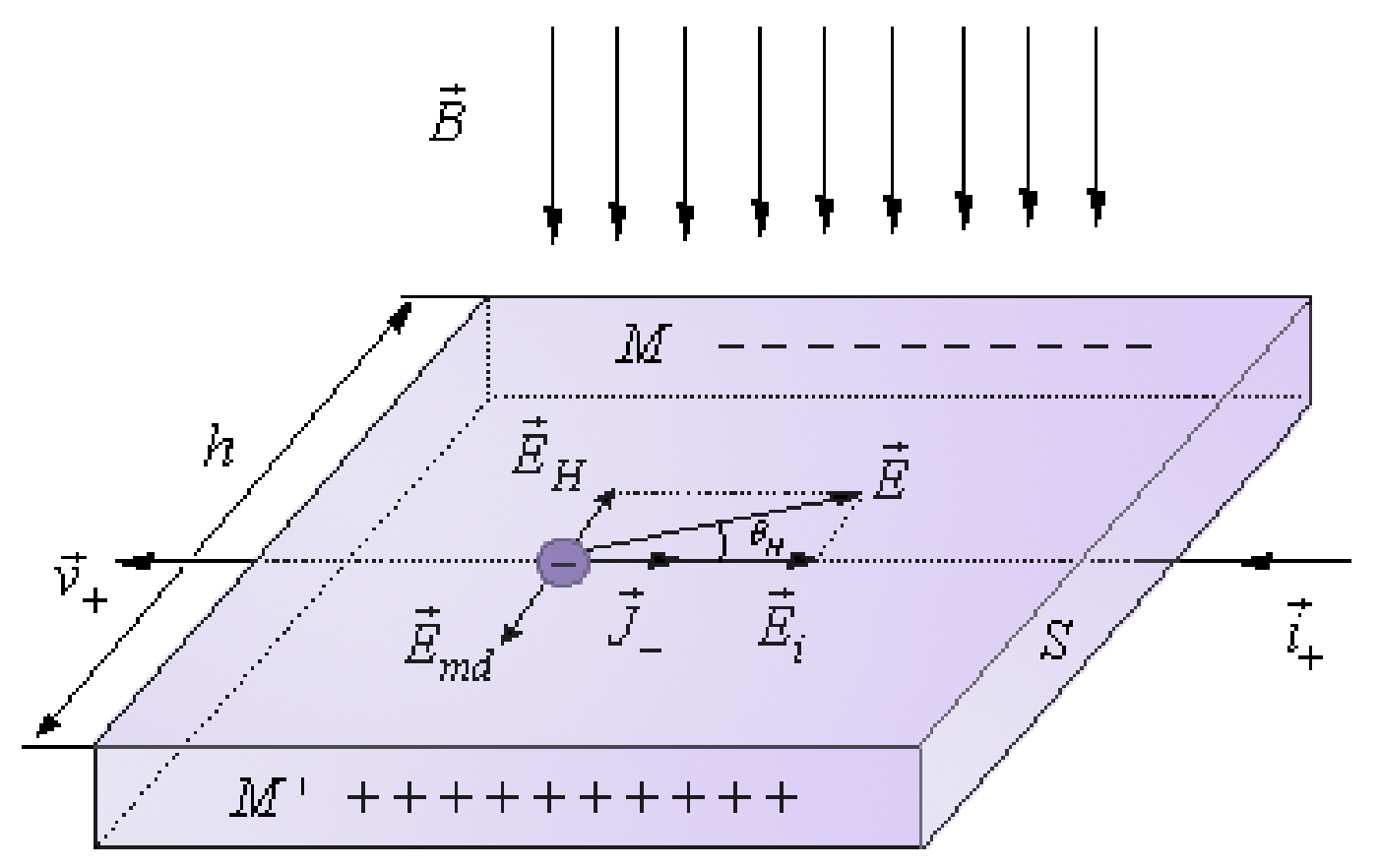

Fig. 16 The Hall effect in metal lamina with monotype carriers of negative charges (electrons). Modified from Zlatev (1972). 
We examine metal lamina in which under the action of electric field $\vec{E}_{i}$ there is current $i_{-}$from electrons with mean velocity $\vec{v}_{-}=$const. We have constant magnetic field with induction $\vec{B}=$ const, whose lines are perpendicular to the metal lamina. The vector of velocity $\vec{v}_{-}$is collinear with the vector of the electron current $\vec{i}_{-}$. The vectors $\vec{i}_{-}$and $\vec{B}$ define electrodynamic field $\vec{E}_{m d}$ by:

$$
\vec{E}_{m d}=\vec{V}_{-} \times \vec{B}
$$

The electromagnetic force $\vec{F}_{M}$, which acts upon $\mathrm{N}$ moving electrons, is:

$$
\vec{F}_{M}=e N \vec{v}_{-} \times \vec{B}
$$

The electromagnetic force $\vec{F}_{M}$ has the opposite direction of $\vec{E}_{m d}$, thus the side M is polarized negatively, while $M^{\prime}$ is polarized positively. Between $M^{\prime}$ and $M$ there is excited potential electric field with intensity $\vec{E}_{H}$. The vector $\vec{E}_{H}$ has the opposite direction of $\vec{E}_{m d}$. The respective force $\vec{F}_{H}$ is defined as:

$$
\vec{F}_{H}=e N \vec{E}_{H}
$$

is the opposite of $\vec{F}_{M}$. The polarization between $\mathrm{M}^{\prime}$ and $\mathrm{M}$ is over when the two forces $\vec{F}_{M}$ and $\vec{F}_{H}$ get equal. The current is still flowing but the vector $\vec{j}_{-}$and the resultant field $\vec{E}$ are no more collinear, instead they form the Hall angle $\theta_{H}$. Then the Ohm's law $\vec{J}_{-}=\gamma \vec{E}$ is not valid ( $\gamma$ is the specific conductivity of the metal).

The calculation of $\theta_{H}$ is simpler when we have monotype current carriers (in our case electrons). If we have equilibrium

$$
\vec{F}_{M}+\vec{F}_{H}=0
$$


Taking into account the geometry of the Hall effect in the metal lamina we can write:

$$
\begin{aligned}
& \vec{E}_{H}=-\vec{v}_{-} \times \vec{B}=\vec{B} \times \vec{v}_{-} \\
& \operatorname{tg} \theta_{H}=\frac{E_{H}}{E_{i}}=-\frac{v_{-}}{E_{i}} B \\
& \theta_{H}=\operatorname{arctg}_{n} B
\end{aligned}
$$

where

$$
k_{n}=-\frac{v_{-}}{E_{i}}
$$

$k_{n}$ is called electron mobility in the metal lamina. If we suppose that the electric polarizing field $\vec{E}_{H}$ is constant the electric voltage $V_{H}$ between $\mathrm{M}^{\prime}$ and $\mathrm{M}$ is:

$$
V_{H}=\vec{E}_{H} h=\vec{V}_{-} \times \vec{B} \cdot \sin \alpha \cdot h
$$

where $\alpha$ is the angle between the vectors $\vec{B}$ and $\vec{V}_{-}$(if $\alpha=90^{\circ}$ then $\sin \alpha=1$ ). The condition $\vec{E}_{H}=$ const is fulfilled if $\vec{v}_{-}=$const and $\vec{B}=$ const. The current density $\vec{j}$ through the surface $S$ of the lamina is:

$$
\begin{aligned}
\vec{J}_{-} & =\frac{i_{-}}{S}=v_{-} \text {en } \\
v_{-} & =R_{H} J_{-} \\
R_{H} & =\frac{1}{\text { en }}
\end{aligned}
$$

where $n$ denotes the number of the elementary charges $e$ in unity volume, and $R_{H}$ is called Hall resistance. $R_{H}$ can thus be measured to find the density of carriers in the material. 
After substitution we find the voltage between the two sides $M$ ' and $M$ to be:

$$
V_{H}=R_{H} J_{-} B h
$$

Often this transverse voltage is measured at fixed current and the Hall resistance recorded. It can easily be seen that this Hall resistance increases linearly with magnetic field.

The Quantum Hall effect (QHE) is phenomenon occurring at low temperatures (millikelvin range) and when strong magnetic fields (1-15 tesla) are applied upon semiconductors. In the absence of magnetic field the density of states in $2 \mathrm{D}$ is constant as a function of energy, but in field the available states clump into Landau levels separated by the cyclotron energy, with regions of energy between the Landau levels where there are no allowed states. As the magnetic field is swept the Landau levels move relative to the Fermi energy. When the Fermi energy lies in a gap between Landau levels electrons cannot move to new states and so there is no scattering. Thus the transport is dissipationless and the resistance falls to zero (Leadley, 1997).

It was supposed (Porter, 2003) that in neurons there is analogous situation where the local magnetic field produced by the neuronal currents acts upon the microtubules and that anyons could be formed via QHE. However the idea that the local magnetic field generated by the neuronal cytoplasmatic currents inputs information to the brain microtubules via classical Hall effect or QHE and that this process is linked to our conscious experience is disproved by the calculations. It is clear that local magnetic flux density varying from zero to $10^{-7} \mathrm{~T}$ makes the idea untenable, because the brain is exposed to stronger magnetic fields and no direct effects on consciousness are registered. It is interesting that strong magnetic fields reaching flux density of $1 \mathrm{~T}$ are used in the fMRI imaging. The visualization of the brain with fMRI however has no impact on consciousness. 


\section{Microtubule lattice structure}

Hameroff (1998a, 1998b, 2003a, 2003b) claims in the "Orch OR list of testable predictions" that the cortical dendrites should contain largely $13 \mathrm{~A}$ lattice microtubules compared to $13 \mathrm{~B}$ lattice microtubules, because $13 \mathrm{~A}$ lattice microtubules are preferable for information processing (Tuszynski et al., 1995).

There were experimental data that in vivo assembled microtubules have $B$ lattice obtained via x-ray scattering by Mandelkow, E. et al. (1977) and electron microscopy and image reconstruction of microtubules by Mandelkow, E.M., et al. (1977); Crepeau et al. (1978); McEwen \& Edelstein (1980). However with the use of recombinant motor proteins (kinesin heads) Song \& Mandelkow (1993) decorated microtubules and doublets showing that all brain microtubules have a $B$ lattice. They questioned the possibility A lattice to exist at all in vivo and presented data favoring the thesis that all in vivo assembled microtubules have predominantly $B$ lattice. This meant that microtubules with 13 protofilaments, the most abundant in vivo, must have a seam where lateral contacts involve heterologous subunits. Such seam has been directly visualized, both in vivo and in vitro, using freeze-fracture replicas (Kikkawa et al., 1994). It is strange that Hameroff (2003b) still insists on 13A lattice importance and defends experimentally disproved position. Maybe in the near future explanation of what makes the seam in the 13B microtubules biologically important will be found.

Some of the interesting properties of the 13B lattice is that the transition from ordered to paraelectric phase happens at higher temperatures than the $13 \mathrm{~A}$ lattice because of the existing seam. Another crucial difference is when MAP attachment to microtubules is considered. MAPs attach themselves to microtubules in periodic patterns and their function is to stabilize the assembly properties of the microtubule by preventing the conformational change of the tubulin dimer to which it is attached. This may be understood by viewing the fixed 
conformation dipoles as seeds of order. Also unlike the lattices that consist of even number of protofilaments (12 or 14) in the 13B lattice to the antiferroelectric ground state can correspond two different values of polarization: - $1 / 13$ when all dipoles in 6 rows point up and all dipoles in 7 rows point down and $+1 / 13$ when all dipoles in 7 rows point up and all dipoles in 6 rows point down. The computational simulations (Trpisova \& Brown, 1998) reveal that the $+1 / 13$ or $-1 / 13$ polarization depends on the MAP/microtubule ratio.

(a)
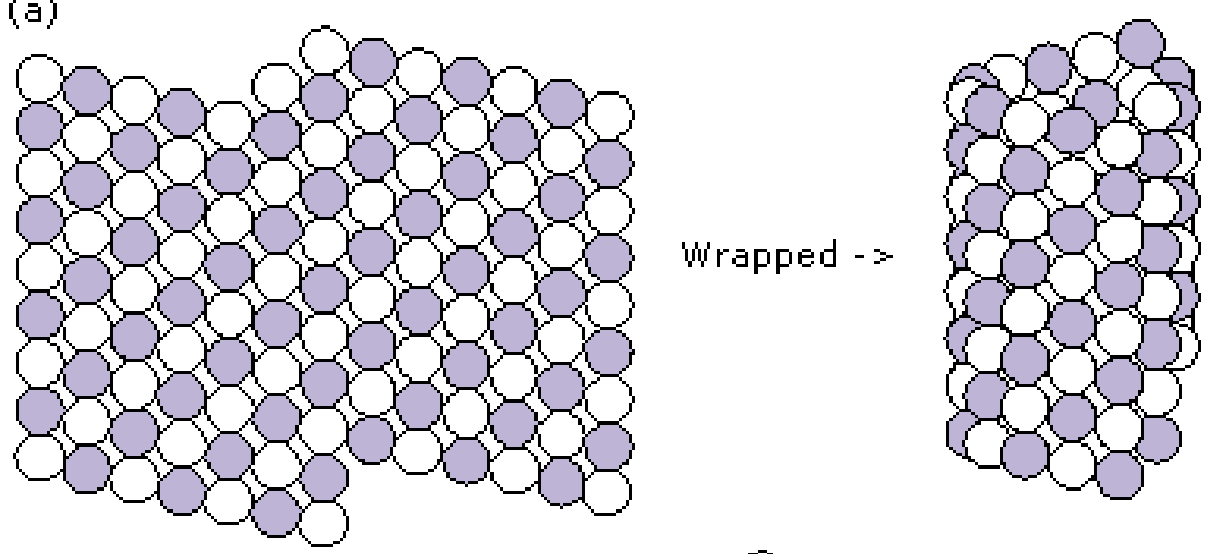

(b)

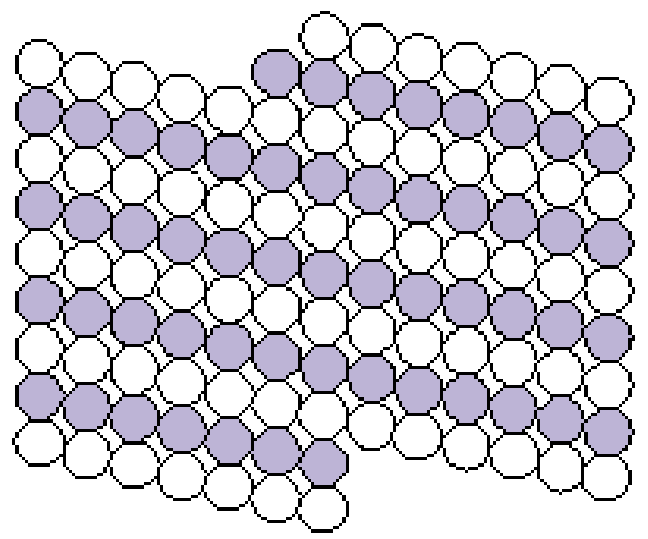

Wrapped $->$ $\alpha$-monomer

B-monomer

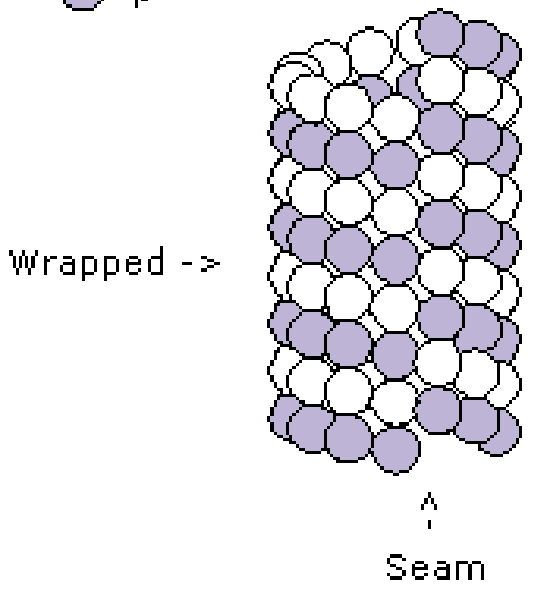

Fig. 17 Unfolded and wrapped $13 A$ and $13 B$ microtubule lattices. Modified from Tuszynski et al. (1998). Brain microtubules in vivo possess $13 B$ lattice (Song \& Mandelkow, 1993; Kikkawa et al., 1994). 
Problems in the ferroelectric model of microtubules

The biophysical aspects of microtubules are the most important issue in direct relationship with microtubular sensitivity to the external electric field (Trpisova \& Brown, 1998; Faber, 2003; Brown \& Tuszynski, 2003). Brown \& Tuszynski (1999) model the microtubules as ferroelectric cylinders and consider that the tubulin dimer has dipole moment that could be in up and down position. In the up position is supposed that the dipole is collinear with the z-axis however when it is in down position the dipole forms angle $\phi \sim 29^{\circ}$ with the z-axis. The ferroelectric model is considered to be improved version of the cellular automaton model developed by Hameroff et al. (1988) where a discrete charge is associated with each tubulin dimer and can either be localized in the top monomer ( $\alpha$ state) or bottom monomer ( $\beta$ state) (Tuszynski et al., 1998).

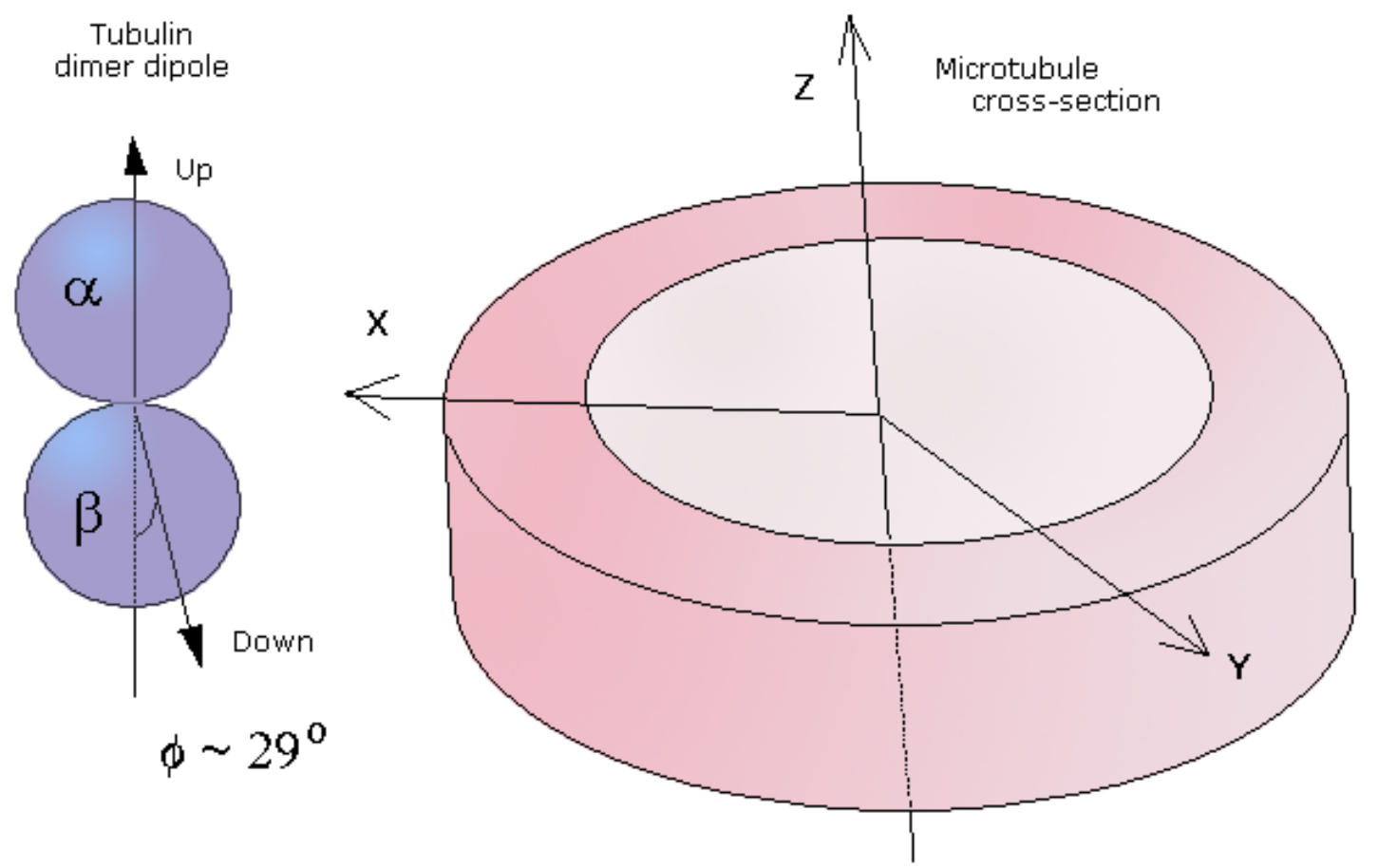

Fig. 18 Dipole moment of the tubulin dimer as considered in the original paper of Brown \& Tuszynski (1999): (a) tubulin dimer dipole; (b) microtubule cross-section with attached coordinate system. 
The developed ferroelectric model however is based upon biologically flawed assumptions and leads to problematic results. The conformational states that are considered to represent single bits (qubits) designated with $\alpha$ and $\beta$, or up and down, are indeed boundary conformations that trigger assembly <-> disassembly of the microtubules, therefore cannot be used for computation by stable microtubule. That's why the calculations show effectiveness of the electric field only when it exceeds $10^{5} \mathrm{~V} / \mathrm{m}$ in the cytoplasm (biological absurd, the microtubules decompose at $2 \times 10^{3} \mathrm{~V} / \mathrm{m}$ as shown by Stracke et al. (2001).

The change in the dipole moment of the tubulin dimer is supposed to be result from electron hopping between the $\alpha$ - and $\beta$-tubulin and is not associated with mechanical rotation of the dimer. Brown \& Tuszynski (2003) investigate the biological electron conductance of microtubules and the associated electron hopping when electric field is applied. The energy value for intra-dimer hopping is estimated to be $\sim 0.4 \mathrm{eV}$ and for the inter-dimer hopping $\sim 1.0 \mathrm{eV}$. The microtubule is not insulator but its conductivity depends on the lattice geometry (13B lattice has lower resistance) and the boundary conditions (whether the microtubule is wrapped up or not). The situation is compared to carbon nanotubes, where particular sets of boundary conditions produce a semi-conducting nanotube while the appropriate choice of wrapping the nanotube gives rise to metallic conduction (Dresselhaus et al., 1996).

The intraneuronal electric field however could not supply the needed energy for the electron hopping. The work W (respectively the energy needed) for intra-dimer hopping could be assessed from the formula:

$$
W=\vec{F} \cdot \vec{l}=\vec{E} \cdot q \cdot \vec{l}
$$


In the model of Brown \& Tuszynski (2003) the assessed value is $\mathrm{W}=0.4 \mathrm{eV}$ $\left(6.4 \times 10^{-20} \mathrm{~J}\right)$, the electron charge is $\mathrm{e}=1.6 \times 10^{-19} \mathrm{C}$ and the hopping distance is approximately $4 \mathrm{~nm}$. Thus we find that the needed electric intensity of the field is:

$$
\vec{E}=\frac{W}{q \cdot \vec{l}}=\frac{6.4 \times 10^{-20} \mathrm{~J}}{1.6 \times 10^{-19} \mathrm{C} \times 4 \times 10^{-9} \mathrm{~m}}=10^{8} \mathrm{~V} \cdot \mathrm{m}^{-1}
$$

In the framework of the same ferroelectric model Trpisova \& Brown (1998) predict that the electric fields inside cell wouldn't have an effect on the dipolar ordering in microtubules. The electric fields start to be effective when they exceed intensity $10^{4}-10^{5} \mathrm{~V} / \mathrm{m}$. The same result implying low effectiveness of the existing electric field is presented also in another papers (Tuszynski et al., 1997b; 1998; Brown \& Tuszynski, 1999). Indeed the intracellular electric field is $1-10 \mathrm{~V} / \mathrm{m}$ (Jaffe \& Nuccitelli, 1977) and in vitro studies showed that if the electric field exceeds $2 \times 10^{3} \mathrm{~V} / \mathrm{m}$ the microtubules are destructed Stracke et al. (2001; 2002).

The energy for the electron hopping between the $\alpha$ - and $\beta$-tubulin could be derived from GDP molecule hydrolysis attached to $\beta$-tubulin. GDP hydrolysis releases approximately $0.4 \mathrm{eV}$ per molecule and is accompanied by a conformational change (Audenaert et al., 1989). This change has been modeled as resulting in a $27^{\circ}$ angle (Melki et al., 1989) between the original line connecting the centers of the $\alpha$ - and $\beta$-monomers and the new centre-to-centre line. These two conformational states and their associated dipole moments have been proposed as the basis for a binary system for information storage and manipulation (Hameroff \& Watt 1982; Hameroff, 1987; 1998a, 1998b; Hameroff et al., 1988; 1992; Smith et al., 1984; Rasmussen et al., 1990; Mavromatos et al., 2002; Mershin et al., 1999; Mershin, 2003; Schuessler et al., 2003). However this model is flawed because the associated conformational change $\alpha<->\beta$ is not biologically useful for computation - leads to microtubular destabilization (Tran et al., 1997) and indeed is linked to assembly <-> disassembly i.e. with microtubule construction <-> destruction. 
GTP hydrolysis and dynamic instability

The main difference between $\alpha$ - and $\beta$-tubulins is in binding GTP. $\alpha$-bound GTP is effectively sequestered - not exchanged and not hydrolyzed - but $\beta$-bound GTP is labile - exchangeable in the free dimer and hydrolyzed to (non-exchangeable) guanosine diphosphate (GDP) in the protofilament (Weisenberg et al., 1976). A significant amount of the free energy of this hydrolysis goes into the microtubule via a conformational change of the tubulin dimer (Caplow et al., 1994). Although the hydrolysis reaction is closely coupled to microtubule assembly (Carlier \& Pantaloni, 1981; Stewart et al., 1990), its consequence is to destabilize the structure. Experiments indicate that unhydrolyzed GTP-tubulin is limited to the last layer of subunits at the end of a microtubule (Voter et al., 1991; Walker et al., 1991; Drechsel \& Kirschner, 1994). The usual interpretation is that this layer acts as a "GTP-cap", keeping an otherwise unstable microtubule intact (Mitchison \& Kirschner, 1984).

Microtubule dynamic instability arises from the hydrolysis of GTP bound to the $\beta$-monomer of the tubulin dimer. The released energy of $0.4 \mathrm{eV}$ from GTP-hydrolysis triggers a conformational change in the tubulin molecule (Hyman et al., 1992) that eventually destabilizes the aggregate (Tran et al., 1997) and causes microtubule disassemble into protofilaments of GDP-bound tubulin that curve away from the microtubule axis. Fygenson (2001) suggests that the unfolding of $\mathrm{N}$-terminal domain called entropic bristle domain (EBD) of the tubulin molecule localized in the microtubule interior leads to disassembly of the microtubule into protofilaments. It is shown that such a change can destabilize the aggregate in a manner consistent with structural data (Mandelkow et al., 1991). The hypothesis not only explains the hydrolysis-associated change in microtubule supertwist (Hyman et al., 1995) but also provides a unifying explanation for the effects of temperature (Fygenson et al., 1994) and glycerol (Fygenson, 1995) on microtubule disassembly rates. 
A.
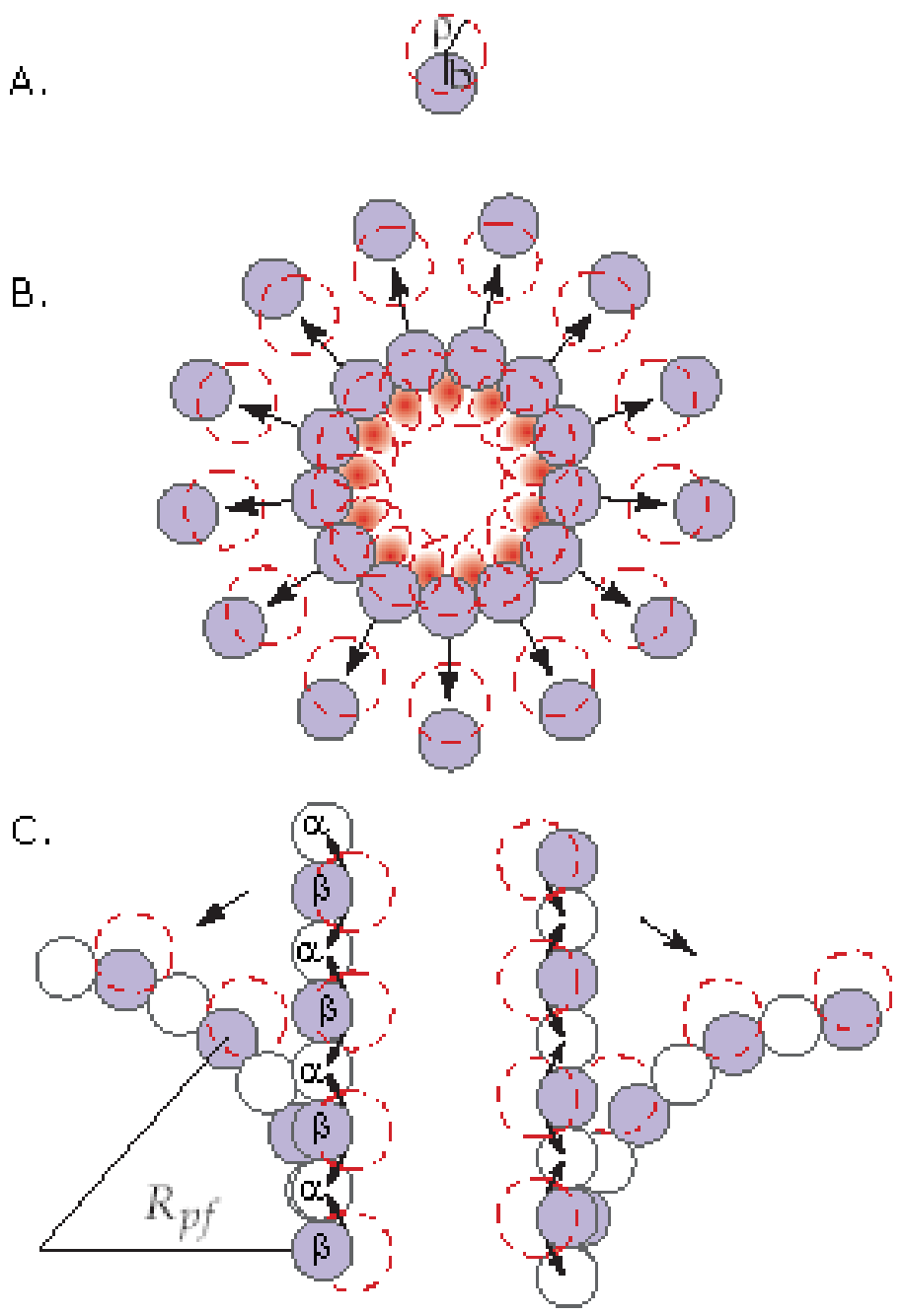

Fig. 19 B-tubulin entropic bristle domain (EBD) regulated microtubule disassembly. A) Side view of a single $\beta$-tubulin monomer (purple) of radius $b$, with an EBD (red) of characteristic size $\rho$. B) Cross-section of a microtubule cut along a row of $\beta$-tubulins. Shading (red) indicates regions of overlap where EBDs sterically hinder one another. Radial expansion of the aggregate releases EBDs from confinement at the expense of lateral bonds. C) Side view of a pair of protofilaments oriented as in a microtubule. For simplicity, the a-tubulin monomers (white) are drawn contacting the $\beta$-tubulin monomers (purple) equidistant on either side of the EBD (red). Note the slight overlap of the EBDs with the a-tubulin spheres. $A$ radius of curvature $R_{p f}$ eliminates the overlap. Modified from Deborah Fygenson (2001). 
At the ends of growing microtubules, protofilaments of different lengths are straight and closely associated. In shortening microtubules, protofilaments separate from one another and curl back, away from the microtubule axis, forming characteristic blunt "blossoms" at the microtubule ends (Tran et al., 1997; Muller-Reichert et al., 1998). This observation is the basis for comments about the tubulin dimer adopting a "curved" or "kidney-bean shaped" conformation, held under tension in the microtubule lattice (Tran et al., 1997; Downing \& Nogales, 1998). Localization of the hydrolyzed nucleotide at the inter-dimer interface is highly suggestive, but which and how residues shift to generate tension and weaken lateral bonds is an important subject of active research (Davis et al., 1994; Sage et al., 1995).

The tubulin conformational states are multiple, with different energy barrier height between them. Therefore we could conclude from the presented mechanism of microtubule assembly <-> disassembly that the computational states (representing bits or qubits) which a stable non-centrosomal neuronal microtubule could adopt must be well chosen, so that to have both stable microtubule structure and biological importance of the output tubulin states. Thus it is obvious that the suggested electron hopping and the considered GTP-hydrolysis linked tubulin dimer transitions (Hameroff, 2003a; 2003b; Tuszynski, 2003; Mershin, 2003) having energy barrier of $0.4 \mathrm{eV}$ are used in biologically improper way in the constructed models to represent bits (or qubits).

The importance of the water microenvironment

NMR evidence (Cope, 1975; Hazelwood et al., 1969; 1974) strongly indicates that cell water possesses more structure than liquid water, and that much of the $\mathrm{Na}^{+}$and $\mathrm{K}^{+}$in the cell is not free in aqueous solution, but is associated with charged sites on macromolecules (Cope, 1975). Therefore, complexed $\mathrm{Na}^{+}$and $\mathrm{K}^{+}$cations have been compared to valence electrons in solid conductors and free 
cations to conduction band electrons. With activation energy barriers and solid-liquid interfaces present in the cell, the liquid state free-cation model of the cell is clearly not applicable. A model based on structured water and associated cations is compatible with thermodynamic evidence.

A substantial part of water molecules in the cell is in the form of hydration water bound to various macromolecules. Yet another large portion exists in the so-called vicinal water form with several exotic properties. It does not have a unique freezing temperature but freezes over the interval of -70 to $-50 \mathrm{C}$ (Tuszynski, 2003). It is a poor solvent for electrolytes but a good one for non-electrolytes, i.e. it behaves as a non-polar solvent. It has a higher viscosity than normal water and exhibits dynamic correlations between individual molecules (Cooke \& Kuntz, 1974; Franks, 1975; Clegg, 1981). Of great interest is the fact that most of the vicinal water surrounds the cytoskeleton (Clegg, 1981). Mascarenhas (1974) demonstrated electret properties of bound water with attendant non-linearity, hysteresis effects and long relaxation times on the order of $1 \mathrm{~s}$ and activation energies of about $7.0-9.0 \mathrm{kcal} / \mathrm{mol}$ have been measured, all of which would tend to indicate the presence of long-range dipolar order leading to the formation of internal electric fields or perhaps collective oscillations of electric fields (Del Giudice et al., 1986).

The microtubule cavities and the vicinal water have been modeled in the framework of the quantum field theory revealing two important phenomena that could take place in the cell: (1) collective infrared photon emission by water molecules known as superradiance (Jibu et al. 1994; 1996; Jibu \& Yasue, 1995; 1997) and (2) Rabi coupling that between the water molecules inside the cavity of the microtubule and the tubulins that build up the microtubule walls (Mavromatos \& Nanopoulos, 1997; 1998; Mavromatos, 2000; Mavromatos et al., 2000, 2002). 
Abdalla et al. (2001) suggested that the vicinal water of the brain microtubules generates electromagnetic sine-Gordon solitons propagating with velocity calculated from the equations:

$$
\begin{aligned}
& v_{0}=V \cdot \frac{2 p}{\pi \hbar} \\
& v_{0}=\frac{2 p}{\pi \hbar} \int E \cdot d z
\end{aligned}
$$

where $z$ is the length of the microtubule, $E$ is the electric field intensity and $p$ is the permanent electric of water $(p=6.2 \times 10-30 \mathrm{Cm})$. After substitution in the equation of the obtained value for $E=10 \mathrm{~V} / \mathrm{m}$ and for microtubule with length $z=50 \mu \mathrm{m}$ we find soliton velocity:

$$
v_{0}=\frac{2 \times 6.2 \times 10^{-30} \mathrm{Cm} \times 10 \mathrm{~V} \cdot \mathrm{m}^{-1} \times 5 \times 10^{-5} \mathrm{~m}}{3.14 \times 1.055 \times 10^{-34} \mathrm{Js}}=18.7 \mathrm{~m} \cdot \mathrm{s}^{-1}
$$

Although the result is an order of magnitude less than the result presented in Abdalla et al. (2001) that calculate $v_{0} \sim 140 \mathrm{~m} / \mathrm{s}$, it shows that fast dissipationless transport of energy could be achieved by collective water molecule dynamics. However the phenomenon per se could not account for explaining the cytoskeletal and microtubule function as being not specific. Indeed proper link with specific protein conformational states must be found if this transfer of energy is supposed to be biologically important. From the presented experimental data and the calculations done in the paper we have seen that $\alpha<\beta \beta$ tubulin 'switches' could not account for biologically feasible model of subneuronal processing of information. This problem is potentially solvable if we focus our attention on the microtubule outer surface and the microtubule <-> MAP and microtubule <-> motor protein interaction. Responsible for the MAP and motor protein control are tubulin C-terminal projections that are highly acidic (hydrophilic) and flexible (allowing multiple conformations with low energy barrier heights). 


\section{Tubulin C-termini biological function}

C-termini of $\alpha$ - and $\beta$ - tubulin known as tubulin "tails" are rich in acidic aminoacid residues (Krauhs et al., 1981; Ponstingl et al., 1981) that is why the C-termini are highly flexible, mobile, susceptible to proteolysis, and exposed to the solvent (Sarkar et al., 2001; Tuszynski, 2003). Because of their intense charge and high flexibility the tubulin C-termini could be sensitive to the local electric field and input the carried by the membrane potentials information. This hypothesis is further supported by the experimental data showing that the highly acidic tubulin C-termini interact with motor proteins and microtubule associated proteins (MAPs). Fujii \& Kozumi (1999) studied the associations of MAPs with tubulin. They found tubulin to undergo many posttranslational modifications at or near the carboxyl termini of the subunits. These carboxyl termini are rich in acidic amino acids and have been shown to be involved in tubulin binding to MAPs. Specifically, they found the MAPs to bind $\alpha$-tubulin at amino acid sequences Lys430-Glu441 and $\beta$-tubulin at amino acid sequences Tyr 422-Gly 434.

Rai \& Wolff (1998) report that the $\beta$-tubulin C-terminus binds microtubuleassociated protein 2 (MAP-2) or tau, whereas the $\alpha$-tubulin $C$ terminus binds these proteins only weakly. The $\alpha$-tubulin C-terminus determines colchicinebinding properties (Mukhopadhyay et al., 1990) despite the fact that the binding site is located on $\beta$-tubulin (Uppuluri et al., 1993; Bai et al., 1996). When colchicine is delivered directly to the brain or cerebrospinal fluid of experimental animals it causes significant cognitive impairments of learning and memory (Bensimon \& Chernat, 1991; Kolasa et al., 1992).

The $\beta$-tubulin C-terminus enhances the polymerization response to vinblastine, while the removal of the $\beta$-tubulin C-terminus abolishes the inhibitory effect of oligoanions on vinblastine-induced polymerization (Rai \& Wolff, 1998). The C-termini of tubulin play a role in the dimer-polymer equilibrium (Sackett et al., 
1985), as attachment points for microtubule-associated proteins and cytoplasmic dynein (Serrano et al, 1984), in the enhancement of the $\mathrm{pH}$ sensitivity of the vinblastine-induced polymerization process, and as possible binding sites for calcium ions (Solomon, 1977).

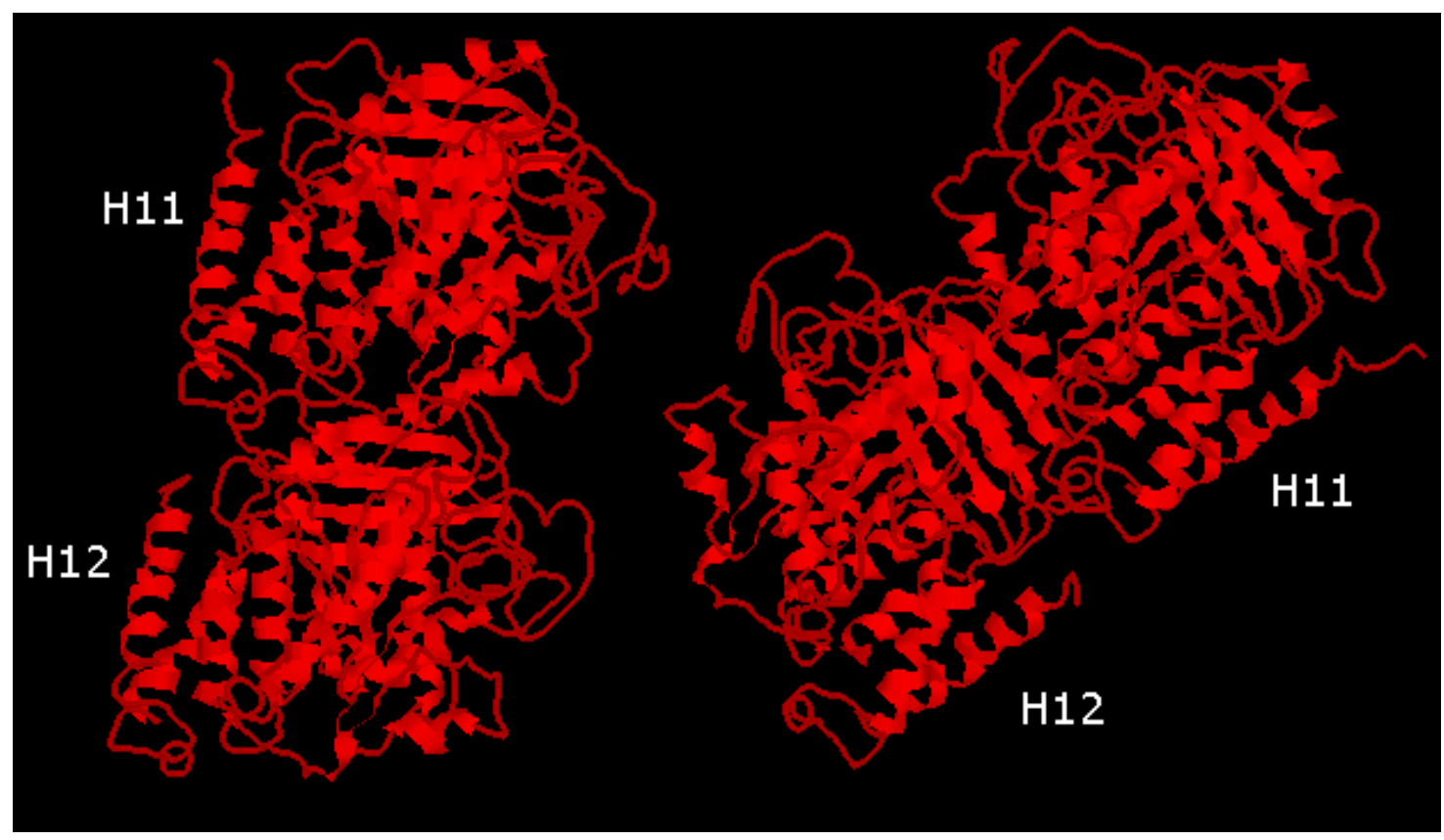

Fig. 20 Tubulin $\alpha / \beta$-dimer structure derived from electron diffraction study (Nogales et al., 1998). Tubulin $\alpha$ and $\beta$ C-termini are shown as helices H11 and H12, respectively. 1TUB.pdb file from The Protein Data Bank is used.

The amino acid sequences encoded by $\beta$-tubulin genes have revealed a high level of overall similarity, but significant divergence between their C-termini (Hall et al., 1985). The pattern of expression of the $\beta$-tubulin genes has been studied in several different human cell lines and has revealed varying levels of and differential expression in different cell lines. It appears that distinct human $\beta$-tubulin isotypes are encoded by genes whose exon size and number has been conserved evolutionarily, but whose pattern of expression may be regulated either coordinately or uniquely (Hall et al., 1985). 
In the variety of C-termini functions is observed and chaperon-like activity (Sarkar et al., 2001). Within the cell exist proteins called "molecular chaperones" i.e. helpers for proper protein folding. They are needed because polypeptides in the cell could fold in multiple ways, some of them biologically useless (aggregation), thus there is always a kinetic competition between the correct folding and the aggregation (Zettlmeissl et al., 1979). The yield of the folded protein will depend upon the relative rate of two processes. Therefore, for successful folding of a protein, chaperones minimize the rate of aggregation.

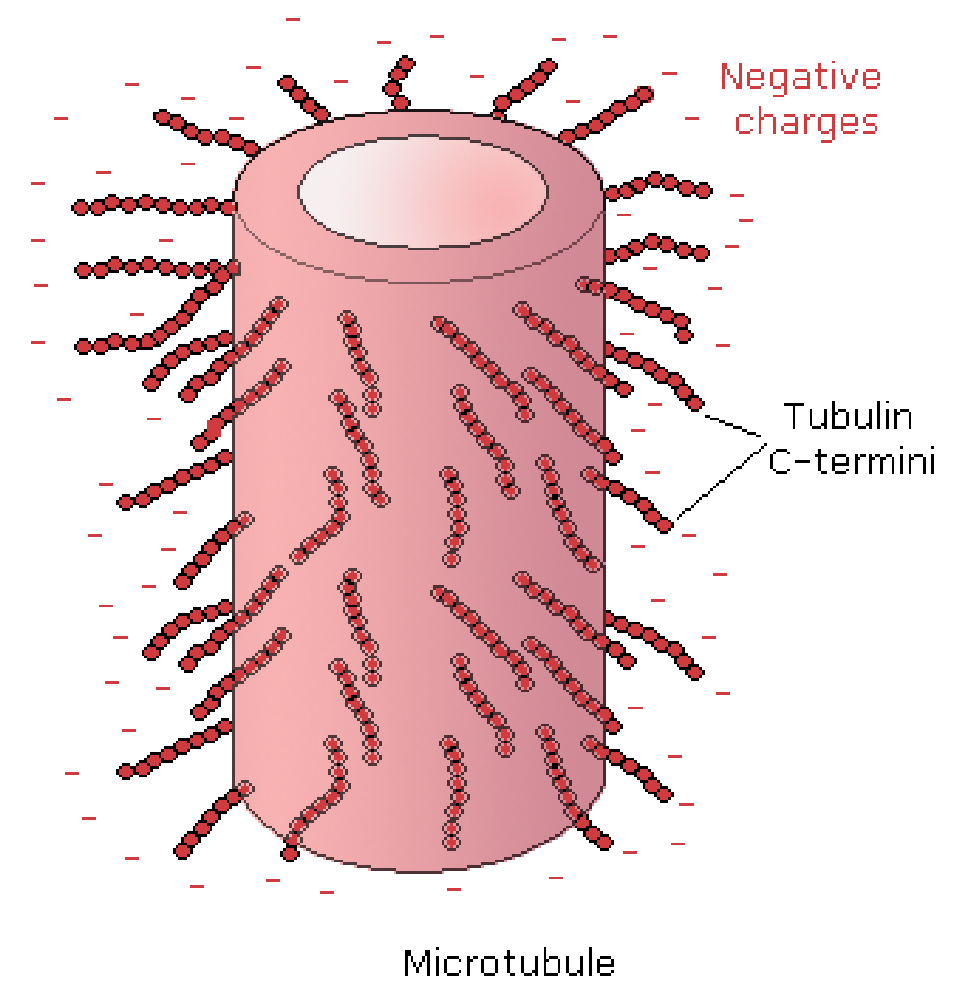

Fig. 21 Tubulin C-termini project from the tubulin subunits and interact with the microtubule-associated structural and motor proteins, cytoplasmic ions ( $\mathrm{Ca}^{++}$) and other charged regulator molecules (colchicine, vinblastine). Additional role for the tubulin tails is chaperon-like activity. 
Post-translational modification of tubulin tails

The tubulin C-termini are prone to intense modification and regulation by different biochemical pathways inside neurons. In mammalian cells, both $\alpha$ - and $\beta$-tubulin occur as seven to eight different genetic variants, which also undergo numerous post-translational modifications (Luduena, 1998; McRae, 1997; Rosenbaum, 2000). The main control of the microtubule function is achieved via covalent modifications (Banerjee, 2002). Indeed in organisms such as the protists that express identical $\alpha$ - and $\beta$-tubulins (Silflow, 1991), post-translational modifications provide the only source of variation. Modifications such as acetylation, palmitoylation, phosphorylation and polyglutamylation are posttranslational modifications found on other proteins; others such as detyrosination and polyglycylation appear to be tubulin specific (McKean et al., 2001).

\section{Tyrosination/detyrosination of $\alpha$-tubulin}

Tyrosination-detyrosination is one of the major posttranslational modifications in which the C-terminal tyrosine residue in $\alpha$-tubulin is added or removed reversibly (Banerjee, 2002). The tubulin tyrosination cycle involves the enzymatic removal of the C-terminal tyrosine residue present on some $\alpha$-tubulin isotypes by a specific carboxy-peptidase, and its subsequent restoration by a tubulin-tyrosine ligase (Idriss, 2000). Although the functional relevance of this modification is not always clear, highly stable microtubules such as those of the axoneme are detyrosinated, and this appears to reflect the length of time the individual $\alpha$-tubulin substrate molecule has spent in a microtubule. Although tyrosination does not alter the assembly activity of tubulin in vitro, these two forms of tubulin have been found to be distributed differently in vivo and are also correlated with microtubule stability (Gundersen et al., 1984). Recent evidence indicates that detyrosination of tubulin can regulate interaction of microtubules with vimentin intermediate filaments by a kinesin-dependent mechanism (Kreitzer et al., 1999). 
Removal of the penultimate glutamate residue from the $\alpha$-tubulin polypeptide produces $\Delta 2$-tubulin, a derivative that is unable to act as a substrate for tubulintyrosine ligase, and this truncated protein is therefore removed from the tyrosination cycle. $\Delta 2$-tubulin is particularly prevalent on microtubular structures such as the axonemes of flagella and cilia and also in mammalian brain cell microtubules.

\section{Polyglutamylation and polyglycylation of both $\alpha$ - and $\beta$-tubulin}

The tubulin modifications polyglutamylation and polyglycylation involve the attachment of oligoglutamyl and oligoglycyl side chains of variable length to specific glutamate residues located near the $\mathrm{C}$-terminus of both $\alpha$ - and $\beta$-tubulin. These side chains can be of considerable length for instance, axonemal tubulin of Paramecium is modified by up to 34 glycyl residues (Bre et al., 1998), and the microtubules of Trypanosoma brucei contain 15 glutamyl residues per $\alpha$-tubulin subunit (Schneider et al., 1997). Polyglutamylation and polyglycylation are particularly associated with stable microtubule structures such as the axonemes of cilia and flagella.

Centriolar microtubules appear to be polyglutamylated but not polyglycylated (Million et al., 1999). Polyglutamylation appears to be critical for the stability of centriole microtubules, since microinjection of monoclonal antibodies specific for polyglutamylated tubulin isotypes, results in the transient disappearance of centrioles in mammalian cells (Bobbinec et al., 1998).

Polyglutamylation also represents the major post-translational modification of axonal tubulin in neuronal cells, where it appears to regulate the differential interaction between microtubules and microtubule-associated proteins (MAPs). For instance, MAPs such as tau and kinesin exhibit optimal binding to tubulin 
modified by $\sim 3$ glutamyl residues, binding affinity decreasing with increased polyglutamyl chain length (Boucher et al., 1994; Larcher et al., 1996). In contrast, increasing polyglutamyl chain length does not appear to affect the binding affinity of MAP1A significantly. Bonnet et al. (2001) suggest that the differential binding of MAPs to polyglutamylated tubulin could facilitate their selective recruitment to distinct microtubule populations and thereby modulate the functional properties of microtubules.

\section{Tubulin tail defects and cerebral pathology}

British type familial amyloidosis is an autosomal dominant disease characterized by progressive dementia, spastic paralysis and ataxia. Amyloid deposits from the brain tissue of an individual who died with this disease have been characterized. Trypsin digestion and subsequent $\mathrm{N}$-terminal sequence analysis yielded a number of short sequences, all of which are tryptic fragments of the C-termini of human $\alpha$ - and $\beta$-tubulin. Consistent with the definition of amyloid, synthetic peptides based on the sequences of these fragments formed fibrils in vitro, suggesting that the $\mathrm{C}$-termini of both $\alpha$ - and $\beta$-tubulin are closely associated with the amyloid deposits of this type of amyloidosis (Ghiso et al., 1996).

\section{Processing of information by brain microtubules}

Taking into account the experimental data from the molecular studies we can construct biologically feasible model of microtubule processing and integration of the electrophysiologic information. Microtubule C-termini are both intensely charged and flexible, so they could undergo conformational transitions separated by relatively low energy barriers when the strength and the direction of the electric field vector changes. They are also biochemically regulated via second messengers coupled to metabotropic receptors (G-protein coupled receptors known also as 7-TM receptors) and have diverse intraneuronal effects. 
Following the Q-mind hypothesis we further could suggest that positive or negative quasiparticles known as solitons could transfer energy between the tubulin tails without dissipation. The solitons are nonlinear waves that do not disperse as they propagate (Dodd et al., 1982; Fordy, 1994) and include kinks, antikinks and different kind of breathers (Dmitriev et al., 1997; 1998a; 1998b; 2000; Miroshnichenko et al., 2000).

We could adopt subneuronal computation in brain microtubules via solitons that are localized excitations propagating in a system with constant velocity and colliding with each other without change in their shapes. During the collision of solitons the solution cannot be represented as a linear combination of two soliton solutions but after the collision solitons recover their shapes and the only result of collision is a phase shift. There are several equations whose solutions produce solitons (Wadati, 2001). One of the best studied is the sine-Gordon model described by Lagrangian:

$$
\mathcal{L}=\frac{1}{2} \frac{\partial^{2} \phi(x, t)}{\partial t^{2}}-\frac{1}{2} \frac{\partial^{2} \phi(x, t)}{\partial x^{2}}-(1-\cos \phi(x, t))
$$

There are known static solutions to the sine-Gordon model:

$$
\begin{aligned}
& \phi=4 \arctan \mathrm{e}^{\mathrm{x}} \\
& \phi=4 \arctan \mathrm{e}^{-\mathrm{x}}
\end{aligned}
$$

that have winding numbers 1 and -1 , respectively. The first solution is known as kink and the second as anti-kink. The kink is an exponentially localized lump of energy centered around $x=0$. Other static solutions can be obtained by translating this solution and moving solutions can be obtained by Lorenz transform. Two of these kinks at different positions can be superimposed and allowed to evolve, they will move away from each other (Houghton, 2000). 
The kink cannot decay even if it is perturbed, this is guaranteed by the winding number. In fact, the sine-Gordon model is peculiar in that a kink-anti-kink configuration does not decay to the vacuum either, in short, if a kink is superimposed with an anti-kink at a different position they will move towards each other but they will not annihilate, they will pass through each other. In fact, there is an oscillating solution known as a breather. This solution has winding number zero but is stable, at least to small perturbations. Kink-anti-kink annihilation is allowed by the conservation of winding number and occurs in similar models. It does not occur in the sine-Gordon model because the sine-Gordon model is integrable.

In the quantized sine-Gordon model the field quanta behave like particles in the normal way, what isn't normal is that the solitons also behave like particles. This means there are two different particle spectra: a soliton spectrum and a spectrum arising out of quantization (Houghton, 2000).

The water molecule superradiance (Jibu et al., 1996; Jibu \& Yasue, 1997) could be part of the tubulin C-termini conformational soliton picture if the conformational states of the tubulin tails are coupled with the dynamics of their hydratation shells. In contrast with the intra-cavital soliton propagation (Abdalla et al., 2001) the C-termini - water molecule solitons are formed on the microtubular surface and could have direct intraneuronal effects such as control of MAP binding and motor protein associated cargo trafficking.

The flexible tubulin tails could explain the electric sensitivity and could account for direct translation of the EPSP and IPSPs into quantum states. The model is attractive because the proposed microtubular solitons could have direct effect upon the presynaptic scaffold protein function and exocytosis as supposed by Georgiev (2003) and because the tubulin tails are prone to extensive biochemical modification - providing link to GPCRs intraneuronal signalling! 

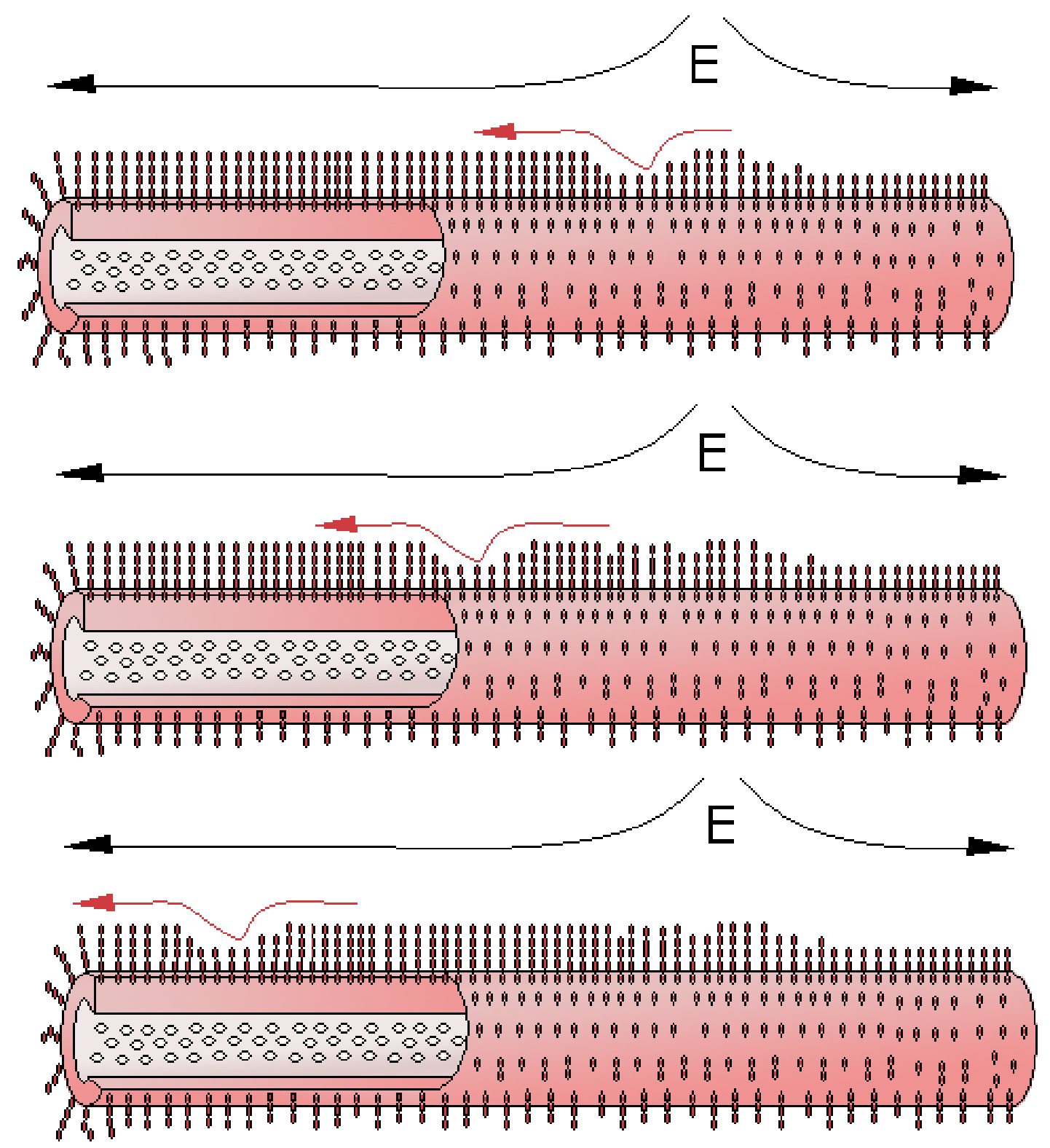

Fig. 22 Propagation of positive quasiparticle (soliton) as local distortion in the conformation of the electronegative tubulin C-termini projecting out of the microtubule. Water molecules (white) are shown inside the microtubule however their collective superradiant behavior on the microtubule outer surface could play essential long-range integrative role between the tubulin tails. The dendritic spine or node of Ranvier that creates local distortion of the electric field is not depicted. 


\section{Elastic and piezoelectric properties of microtubules}

The elasticity of microtubules is another issue of physical relevance, which has been of recent theoretical and experimental interest. Using Gittes procedure (Gittes et al., 1993) to determine flexural rigidity, Mickey \& Howard (1995) determined the Young's modulus of microtubules to be E=1.4 GPa. The value of Young's modulus increases almost three-fold under the stabilizing action of MAP-tau or taxol. The knowledge of this value allowed Sirenko et al. (1996) to model the vibrations of microtubule within a fluid. They found that the microtubule within water can support interface elastic waves with frequencies up to the gigahertz range and that acoustic waves with velocities from 200 to $600 \mathrm{~m} / \mathrm{s}$ are possible.

Another interesting feature of microtubules is that they could have piezoelectric properties (Athenstaedt, 1974). The word piezo is Greek for "push". Crystals, which acquire a charge when compressed, twisted or distorted, are said to be piezoelectric. This provides a convenient transducer effect between electrical and mechanical (elastic) oscillations. The reverse phenomenon is also observed: if an electrical oscillation is applied to piezoelectric crystal, it will respond with mechanical vibrations. The biological importance of the piezoelectric effect in microtubules remains to be assessed.

\section{Discussion}

The local electric and magnetic field strengths were assessed to be varying from $0.01 \mathrm{~V} / \mathrm{m}$ to $10 \mathrm{~V} / \mathrm{m}$ for the electric intensity and from $10^{-10}$ to $10^{-7} \mathrm{~T}$ for the magnetic flux density. This rules out the possibility for the local magnetic field to input sensory information to tubulins. We have seen that if microtubules are intimately linked to our "consciousness" then they should have developed mechanisms for inputting the information carried by the local electric field. 
Here should be noted that the widely quoted "sensitivity" of microtubules to external electric and magnetic fields is indeed (1) arrangement along the field strength lines of the microtubules (Vassilev et al., 1982), (2) electrophoretic mobility (Vater et al., 1998; Stracke et al., 2001; 2002) and (3) use of high intensity magnetic field exceeding 7.6 tesla in order to produce aligned samples of hydrated microtubules suitable for low-resolution $x$-ray fiber diffraction experiments (Bras et al., 1998). All these microtubule-electromagnetic field interactions have no direct biological importance and this "sensitivity" should not be used as proof of in vivo existing such interaction.

In contrast attractive neuromolecular model based upon the tubulin tail function is proposed in which tubulin tails are "sensitive" to local changes of the intraneuronal electric field strength and direction. Positively and negatively charged quasiparticles (solitons) are formed and propagate on the microtubular surface transferring information and energy without dissipation and exhibiting long-range correlations. The tubulin C-termini function is essentially linked both to the electrophysiologic input (fast response) and to biochemical cascades (memorization). Thus the Q-mind hypothesis is presented in "new face" meeting the classical neuroscience.

The conclusion from the presented data is that before starting microtubule quantum modeling it is useful to be acquainted with the specific intracellular microenvironment. The local electric field in the brain cortex is the carrier of information to our mind (Dobelle, 2000), that's why if microtubules are linked to the cognitive processes in brain their interaction with the electric field is crucial and must be taken into account in the future models. 


\section{References}

1. Abdalla, E., Maroufi, B., Melgar, B.C. \& Sedra, M.B. (2001). Information transport by sine-Gordon solitons in microtubules. http://arXiv.org/abs/physics/0103042

2. Akaike, N. \& Moorhouse, A.J. (2003). Techniques: Applications of the nerve-bouton preparation in neuropharmacology. Trends in Pharmacological Sciences 24(1):44-47.

3. Akaike, N. et al. (2002). Focal stimulation of single GABAergic presynaptic boutons on the rat hippocampal neuron. Neurosci. Res. 42, 187-195.

4. Ahmad, F.J., Yu, W., McNally, F.J. \& Baas, P.W. (1999). An essential role for katanin in severing microtubules in the neuron. J. Cell Biol. 145, 301315.

5. Arfken, G. (1985). "Gradient, $\nabla$ " and "Successive Applications of $\nabla$." $§ 1.6$ and 1.9 in Mathematical Methods for Physicists, 3rd ed. Orlando, FL: Academic Press, pp. 33-37 and 47-51.

6. Athenstaedt, H. (1974). Pyroelectric and piezoelectric properties of vertebrates. Ann NY Acad Sci 238: 68-94.

7. Audenaert, R., Heremans, L., Heremans, K. \& Engelborghs, Y. (1989). Secondary structure analysis of tubulin and microtubules with Raman spectroscopy. Biochim Biophys Acta, 996 (1-2):110-115.

8. Bai, R., Pei, X.-F., Boye, O., Getahun, Z., Gover, S., Bekisz, J., Nguyen, N. Y., Brossi, A. \& Hamel, E. (1996). Identification of Cysteine 354 of $\beta$-Tubulin as Part of the Binding Site for the A Ring of Colchicine. J. Biol. Chem. 271, 12639-12645.

9. Baker, N.A., Sept, D., Joseph, S., Holst, M.J. \& McCammon, J.A. (2001). Electrostatics of nanosystems: Application to microtubules and the ribosome. $\quad$ PNAS 98 (18): 10037-10041. http://www.pnas.org/cgi/content/abstract/98/18/10037 
10. Banerjee, A. (2002). Coordination of Posttranslational Modifications of Bovine Brain $\alpha$-Tubulin: Polyglycylation of $\Delta 2$ tubulin. The Journal Of Biological Chemistry 277 (48):46140-46144

11. Bensimon, G. \& Chernat, R. (1991). Microtubule disruption and cognitive defects: effect of colchicine on teaming behavior in rats. Pharmacol. Biochem. Behavior, 38, 141-145.

12. Berman, H.M., Westbrook, J., Feng, Z., Gilliland, G., Bhat, T.N., Weissig, H., Shindyalov, I.N. \& Bourne, P.E. (2000). The Protein Data Bank. Nucleic Acids Research, 28 pp. 235-242. URL: http://www.rcsb.org/pdb/

13. Bobbinec, Y., Khodjakov, A., Mir, L. M., Reider, C. L. \& Bornens, M. (1998). Centriole disassembly in vivo and its effects on centrosome structure and function in vertebrate cells. J. Cell Biol. 143, 1575-1589.

14. Bonnet, C., Boucher, D., Lazereg, S.P., Pedrotti, B., Islam, K., Denoulet, P. \& Larcher, J.C. (2001). Differential binding regulation of microtubuleassociated proteins MAP1A, MAP1B and MAP2 by tubulin polyglutamylation. J. Biol. Chem. 276, 12839-12848.

15. Boucher, D., Larcher, J.-C., Gros, F. \& Denoulet, P. (1994). Polyglutamylation of tubulin as a progressive regulator of in vitro interactions between the microtubule-associated protein tau and tubulin. Biochemistry 33, 12471-12477.

16. Bras, W., Diakun, G.P., Diaz, J.F., Maret, G., Kramer, H., Bordas, J. \& Medrano, F.J. (1998). The Susceptibility of Pure Tubulin to High Magnetic Fields: A Magnetic Birefringence and X-Ray Fiber Diffraction Study. Biophys J, 74(3):1509-1521.

17. Bre, M.-H., Redeker, V., Vinh, J., Rossier, J. \& Levilliers, N. (1998). Tubulin polyglycylation: differential post-translational modification of dynamic cytoplasmic and stable axonemal microtubules in Paramecium. Mol. Biol. Cell 9, 2655-2665.

18. Brown, J.A. \& Tuszynski, J.A. (1999). A Review of the Ferroelectric Model of Microtubules. Ferroelectrics 220 141-156. 
19. Brown, J.A. \& Tuszynski, J.A. (2003). Calculation of the Electrical Conduction by Microtubule Protofilaments, Sheets and Cylinders. Submitted to Physical Review E. http://mitacsgw.phys.ualberta.ca/mmpd/conduction.pdf

20. Caplow, M., Ruhlen, R.L. \& Shanks, J. (1994). The free energy for hydrolysis of a microtubule-bound nucleotide triphosphate is near zero: all of the free energy for hydrolysis is stored in the microtubule lattice. J Cell Biol. 127:779-788.

21. Carlier, M.F. \& Pantaloni, D. (1981). Kinetic analysis of guanosine 5 -triphosphate hydrolysis associated with tubulin polymerization. Biochemistry. 20:1918-1924.

22. Cauller, L. (2003). Fall 2003 NeuroPhysiology Course. http://www.utdallas.edu/ Icauller/

23. Chang, H.T. (1952). Cortical neurons with particular reference to the apical dendrites. Cold Spring Harbor Syrnp. Quant. Biol., 17:189-202.

24. Clay, J.R. (1998). Excitability of the squid giant axon revisited. J.Neurophysiol., 80:903-913.

25. Clegg, J. (1981). Coll. Phenomena, 3, 289.

26. Cooke, R. \& Kuntz, I. (1974). The properties of water in biological systems. Annu. Rev. Biophys. Bioeng. 3, 95-126.

27. Cope, F. (1975). A review of the applications of solid state physics concepts to biological systems. J. Biol. Phys. 3, 1-41.

28. Coss, R.G. \& Globus, A. (1978). Spine stems on tectal interneurons in jewel fish are shortened by social stimulation. Science, 200:787- 790.

29. Crepeau, R.H., McEwen, B. \& Edelstein, S.J. (1978). Proc. Natl Acad. Sci. USA 75, 5006-5010.

30. Davis, A., Sage, C.R., Dougherty, C.A. \& Farrell, K.W. (1994). Microtubule dynamics modulated by guanosine triphosphate hydrolysis activity of $\beta$-tubulin. Science. 264:839-842. 
31. Del Giudice, E., Doglia, S., Milani, M., \& Vitiello, G. (1986). Modern Bioelectrochemistry. London: Plenum Press.

32. Diamond, J., Gray, E.G. \& Yasargil, G.M. (1970). The function of the dendritic spines: a hypothesis. In P. Anderson and J. K. S. Jansen (Eds.), Excitatory Synaptic Mechanisms, Universitetsforlaget, Oslo, pp. 213-222.

33. Dmitriev, S.V., Nauman, L.V., Wusatowska-Sarnek, A.M. \& Starostenkov, M.D. (1997). Generation and Annihilation of Dislocations in the Discrete Frenkel-Kontorova Model Phys. Stat. Sol. (b) 201, 89

34. Dmitriev, S.V., Shigenari, T., Vasiliev, A.A. \& Miroshnichenko, A.E. (1998a). Effect of discreteness on a sine-Gordon three-soliton solution Physics Letters A (246) 1-2 pp.129-134

35. Dmitriev, S.V., Abe, K., Shigenari, T., Vasiliev, A.A. \& Miroshnichenko, A.E. (1998b). Strongly Inelastic Collision of Soliton in Weakly Perturbed Sine-Gordon Equation Meeting abstracts of the Physical Society of Japan, September 25-29, Volume 53, Issue 2, Part 2, P. 278.

36. Dmitriev, S.V., Miyauchi, T., Abe, K. \& Shigenari, T. (2000). Kink-breather solution in the weakly discrete Frenkel-Kontorova model Phys. Rev. E 61, 5880

37. Dobelle, W.H. (2000). Artificial Vision for the Blind by Connecting a Television Camera to the Visual Cortex. ASAIO Journal 2000; 46:3-9. http://www.artificialvision.com/download/article.pdf

38. Dodd, R.K., Eilbeck, J.C., Gibbon, J.D. \& Morries, H.C. (1982). Solitons and Nonlinear Wave Equations. Academic Press, London

39. Downing, K.H. \& Nogales, E. (1998). Tubulin and microtubule structure. Curr Opin Cell Biol. 10:16-22.

40. Drechsel, D.N. \& Kirschner, M.W. (1994). The minimum GTP cap required to stabilize microtubules. Curr Biol. 4:1053-1061.

41. Dresselhaus, M.S., Dreselhaus, G. \& Eklund, P.C. (1996). Science of fullerenes and carbon nanotubes. San Diego: Academic Press. 
42. Eichler West, R.M., De Schutter, E. \& Wilcox, G.L. (1998). Using Evolutionary Algorithms to Search for Control Parameters in a Nonlinear Partial Differential Equation. Institute for Mathematics and Its Applications volume on Evolutionary Algorithms and High Performance Computing. Springer-Verlag.

http://bbf-

www.uia.ac.be/publications/ima001/TNB_ima1.shtml

43. Erickson, H.P. \& Stoffler, D. (1996). Tubulin rings are universal polymers of the tubulin family $-\alpha / \beta, \gamma$ and FtsZ. Journal of Cell Biology, 135:5-8

44. Faber, J. (2003). Information Processing in Brain Microtubules. In Proceedings Quantum Mind 2003: "Consciousness, Quantum Physics and the Brain", Convention Center and Leo Rich Theater, Tucson, Arizona.

45. Feldman, M.L. \& Peters, A. (1978). The forms of non-pyramidal neurons in the visual cortex of the rat. Journal of Comparative Neurology, 179, 761794.

46. Fiala, J. \& Harris, K. (1999). Dendrite Structure. In Dendrites, edited by G.Stuart, N.Spruston \& M.Hausser. http://synapses.bu.edu/lab/fiala/dendrites.pdf

47. Fleshman, J.W., Segev, I. \& Burke, R.B. (1988). Electrotonic architecture of type-identified $\alpha$-motoneurons in the cat spinal cord. Journal of Neurophysiology,60(1):60-85.

http://jn.physiology.org/cgi/content/abstract/60/1/60

48. Fordy, A.P. (1994). A historical introduction to solitons and Backlund transformations. In A.P. Fordy and J.C. Wood, editors, Harmonic Maps and Integrable Systems, pages 7-28. Vieweg, Wiesbaden. http://www.amsta.leeds.ac.uk/Pure/staff/wood/FordyWood/fordy.ps

49. Franks, F. (1975). Water, A Comprehensive Treatise. London: Pergamon.

50. Frick, P., Khripchenko, S., Denisov, S., Sokoloff, D. \& Pinton, J.F. (2003). Effective magnetic permeability of a turbulent fluid with macroferroparticles. EPJ manuscript. http://www.enslyon.fr/ pinton/ARTICLES/mhd_SB_EPJB.pdf 
51. Fujii \& Koizumi (1999). Identification of the binding region of basic calponin on $\alpha$ - and $\beta$-tubulins. Japanese Biochemical Society:125(5), 869-875.

52. Fygenson, D. K. (1995). Microtubules: the rhythm of growth and evolution of form. Ph.D. Princeton University, Princeton.

53. Fygenson, D. (2001). A Unifying Hypothesis for the Conformational Change of Tubulin. http://arxiv.org/pdf/physics/0101078

54. Fygenson, D. K., Braun, E. \& Libchaber, A. (1994). Phase diagram of microtubules. Physical Review. E. Statistical Physics, Plasmas, Fluids, and Related Interdisciplinary Topics. 50:1579-1588.

55. Georgiev, D. (2003). On the dynamic timescale of mind-brain interaction. In Proceedings Quantum Mind 2003: "Consciousness, Quantum Physics and the Brain", Convention Center and Leo Rich Theater, Tucson, Arizona.

56. Ghiso, J., Plant, G.T., Levy, E., Wisniewski, T. \& Baumann, M.H. (1996). C-terminal fragments of $\alpha$ - and $\beta$-tubulin form amyloid fibrils in vitro and associate with amyloid deposits of familial cerebral amyloid angiopathy, British type. Biochem. Biophys. Res. Commun. 219: 238- 242

57. Gittes, F., Mickey, E. \& Nettleton, J. (1993). Flexural rigidity of microtubules and actin filaments measured from thermal fluctuations in shape. J. Cell Biol., 120:923

58. Gundersen, G.G., Kalnoski, M.H. \& Bulinski, J.C. (1984). Distinct populations of microtubules: tyrosinated and nontyrosinated $\alpha$-tubulin are distributed differently in vivo. Cell 38(3):779-789.

59. Hall, J.L., Gilmartin, M.E., Cowan, N.J. \& Lewis, S.A. (1985). Three expressed sequences within the human $\beta$-tubulin multigene family each define a distinct isotype. J. Mol. Biol. 182: 11- 20

60. Hameroff, SR. (1987). Ultimate Computing: Biomolecular Consciousness and Nanotechnology. Elsevier-North Holland, Amsterdam. 
61. Hameroff, S. (1998a). Funda-mental geometry: The Penrose-Hameroff Orch OR model of consciousness. In: The geometric universe - Science, geometry and the work of Roger Penrose. Eds. S.A. Huggett, L.J. Mason, K.P. Tod, S.T. Tsou, and N.M.J. Woodhouse. Oxford Press, Oxford, U.K. pp 135-160

62. Hameroff, S. (1998b). Quantum computation in microtubules? The Penrose-Hameroff 'Orch OR' model of consciousness. Philosophical Transactions of the Royal Society A (London) 356:1869-1896

63. Hameroff, S. (2003a). Quantum biology: At what levels are quantum effects relevant? In Proceedings Quantum Mind 2003: "Consciousness, Quantum Physics and the Brain", Convention Center and Leo Rich Theater, Tucson, Arizona.

64. Hameroff, S. (2003b). Testing the biological basis of the Orch-OR model of consciousness. In Proceedings Quantum Mind 2003: "Consciousness, Quantum Physics and the Brain", Convention Center and Leo Rich Theater, Tucson, Arizona.

65. Hameroff, S.R. \& Watt, R.C. (1982). Information processing in microtubules. J Theor Biol 98:549-561

66. Hameroff, S.R., Rasmussen, S. \& Mansson, B. (1988). Molecular automata in microtubules: basic computational logic of the living state? In Artificial life. New York: Addison-Wesley.

67. Hameroff, S.R., Dayhoff, J.E., Lahoz-Beltra, R., Samsonovich, A. \& Rasmussen, S. (1992). Conformational Automata in the Cytoskeleton: models for molecular computation. IEEE Computer 25(11):30-39.

68. Hazelwood, C., Nichols, B., \& Chamberlain, N. (1969). Evidence for the existence of a minimum of two phases of ordered water in skeletal muscle. Nature, 222, 747-750.

69. Hazelwood, C., Chang, D., Nichols, B., \& Woessner, D. (1974). NMR transverse relaxation times of water protons in skeletal muscle. Biophys. J. $14,583-606$. 
70. Hodgkin, A.L. \& Huxley, A.F. (1952a). The components of the membrane conductance in the giant axon of the loligo. J. Physiol., 116:473-496.

71. Hodgkin, A.L. \& Huxley, A.F. (1952b). Currents carried by the sodium and potassium ion through the membrane of the giant axon of loligo. J. Physiol., 116:449-472.

72. Hodgkin, A.L. \& Huxley, A.F. (1952c). The dual effect of membrane potential on sodium conductance in the giant axon of loligo. J. Physiol., 116:497-506.

73. Houghton, C. (2000). The sine-Gordon Kink. Rough notes for Maths 543. http://www.maths.tcd.ie/ houghton/TEACHING/543/543.3.pdf

74. Hyman, A.A., Salser, S., Drechsel, D.N., Unwin, N. \& Mitchison, T.J. (1992). Role of GTP hydrolysis in microtubule dynamics: information from a slowly hydrolyzable analogue, GMPCPP. Mol Biol Cell. 3:1155-1167.

75. Hyman, A.A., Chretien, D, Arnal, I. \& Wade, R.H. (1995). Structural changes accompanying GTP hydrolysis in microtubules: information from a slowly hydrolyzable analogue guanylyl- $(\alpha, \beta)$ - methylene-diphosphonate. J Cell Biol. 128:117-125.

76. Idriss, H.T. (2000). Man to trypanosome: the tubulin tyrosination/ detyrosination cycle revisited. Cell Motil. Cytoskel. 45, 173-184.

77. Jack, J.J.B., Noble, D. \& Tsien, R. (1975). Electric current flow in excitable cells. Oxford.

78. Jaffe, L.F. \& Nuccitelli, R. (1977). Electrical controls of development. Annual Review of Biophysics and Bioengineering 6: 445-476.

79. Jibu, M., Hagan, S., Hameroff, S.R., Pribram, K.H. \& Yasue, K. (1994). Quantum optical coherence in cytoskeletal microtubules: implications for brain function. Biosystems 32: 195-209.

80. Jibu, M., Pribram, K.H. \& Yasue, K. (1996). From conscious experience to memory storage and retrieval: the role of quantum brain dynamics and boson condensation of evanescent photons. International Journal Of Modern Physics B, Vol.10, Nos. 13 \& 14: 1735-1754. 
81. Jibu, M. \& Yasue, K. (1995). Quantum Brain Dynamics: An Introduction. John Benjamins: Amsterdam.

82. Jibu, M. \& Yasue, K. (1997). What is mind? Quantum field theory of evanescent photons in brain as quantum theory of consciousness. Informatica 21, pp. 471-490.

83. Kaplan, W. (1991). "The Gradient Field." §3.3 in Advanced Calculus, 4th ed. Reading, MA: Addison-Wesley, pp. 183-185.

84. Katz, B. (1966). Nerve, Muscle and Synapse. McGraw-Hilt, New York

85. Kellerth, J.O., Berthold, C.H. \& Conradi, S. (1979). Electron microscopic studies of serially sectioned cat spinal $\alpha$-motoneurons. III. Motoneurons innervating fast-twitch (type FR) units of the gastrocnemius muscle. Journal of Comparative Neurology, 184, (4), 755-767.

86. Kikkawa, M., Ishikawa, T., Nakata, T., Wakabayashi, T. \& Hirokawa, N. (1994). Direct visualization of the microtubule lattice seam both in vitro and in vivo. J. Cell Biol. 127, 1965-1971. http://www.jcb.org/cgi/reprint/127/6/1965.pdf

87. Kirischuk, S. et al. (1999). Relationship between presynaptic calcium transients and postsynaptic currents at single GABAergic boutons. Proc. Natl Acad. Sci. USA 96, 7520-7525.

88. Kitaev, A. (1997). Fault-tolerant quantum computation by anyons. http://arxiv.org/abs/quant-ph/9707021

89. Kolasa, K., Jope, R.S., Baird, M.S. \& Johnson, G.V.W. (1992). Alterations of choline acetyltransferase, phosphoinositide hydrolysis, and cytoskeletal proteins in rat brain in response to colchicine administration. Exp. Brain Res., 89, 496-500.

90. Krauhs, E., Little, M., Kempf, T., Hofer-Warbinek, R., Ade, W. \& Ponstingl, H. (1981). Complete amino acid sequence of $\beta$-tubulin from porcine brain. Proc Natl Acad Sci USA 78:4156-4160. 
91. Kreitzer, G., Liao, G. \& Gundersen, G.G. (1999). Detyrosination of tubulin regulates the interaction of intermediate filaments with microtubules in vivo via a kinesin-dependent mechanism. Mol. Biol. Cell 10, 1105-1118.

92. Larcher, J.-C., Boucher, D., Lazareg, S., Gros, F. \& Denoulet, P. (1996). Interactions of kinesin motor domains with $\alpha$ - and $\beta$-tubulin subunits at a $\tau$-independent binding site. Regulation by polyglutamylation. J. Biol. Chem. 271, 22117-22124.

93. Leadley, D.R. (1997). Quantum Hall effect. http://www.warwick.ac.uk/ phsbm/qhe.htm

94. London, M. \& Segev, I. (2001). Synaptic scaling in vitro and in vivo. Nature Neuroscience,4(9):853-854.

http://lobster.Is.huji.ac.il/idan/files/London_Segev_NN2001.pdf

95. Luduena, R. F. (1998). Multiple forms of tubulin: different gene products and covalent modifications. Int. Rev. Cytol. 178, 207-275.

96. Mandelkow. E., Thomas, J. \& Cohen, C. (1977). Microtubule structure at low resolution by x-ray diffraction. Proc. Natl. Acad. Sci. USA 74(8), 3370-3374.

97. Mandelkow. E.M., Mandelkow. E., Unwin, P.N.T. \& Cohen, C. (1977). Nature (London) 265, 655-657.

98. Mandelkow, E.M., Mandelkow, E. \& Milligan, R.A. (1991). Microtubule dynamics and microtubule caps: a time-resolved cryo-electron microscopy study. J Cell Biol. 114:977-991.

99. Mascarenhas, S. (1974). The electret effect in bone and biopolymers and the bound water problem. Ann. NY. Acad. Sci., 238, 36-52.

100. Mavromatos, N.E. (2000). Cell Microtubules as Cavities: Quantum Coherence and Energy Transfer? http://arXiv.org/abs/quant-ph/0009089

101. Mavromatos, N.E. \& Nanopoulos, D.V. (1997). On Quantum Mechanical Aspects of Microtubules. http://arXiv.org/abs/quant-ph/9708003 
102. Mavromatos, N.E. \& Nanopoulos, D.V. (1998). Quantum Mechanics in Cell Microtubules: Wild Imagination or Realistic Possibility? http://arXiv.org/abs/quant-ph/9802063

103. Mavromatos, N.E., Nanopoulos, D.V., Samaras, I. \& Zioutas, K. (1998). Ferroelectrics and their possible involvement in biology. http://arXiv.org/abs/quant-ph/9803005

104. Mavromatos, N.E., Mershin, A. \& Nanopoulos, D.V. (2002). QED-Cavity model of microtubules implies dissipationless energy transfer and biological quantum teleportation. http://arxiv.org/abs/quant-ph/0204021

105. Mayer, M.L. \& Vyklicky, L. Jr. (1989). Concanavalin A selectively reduces desensitization of mammalian neuronal quisqualate receptors. Proc Natl Acad Sci U S A. 86(4):1411-1415.

106. McEwen, B. \& Edelstein, S.J. (1980). Evidence for a mixed lattice in microtubules reassembled in vitro. J. Mol. Biol. 139, 123-145.

107. McKean, P.G., Vaughan, S. \& Gull, K. (2001). The extended tubulin superfamily. Journal of Cell Science 114 (15) 2723-2733

108. McRae, T. H. (1997). Tubulin post-translational modifications: enzymes and their mechanisms of action. Eur. J. Biochem. 244, 265-278.

109. Melki, R., Carlier, M.F., Pantaloni, D., Timasheff, S.N. (1989). Cold depolymerization of microtubules to double rings: geometric stabilization of assemblies. Biochemistry 28, 9143-9152.

110. Mershin, A. (2003). Experimental "quantum brain"? In Proceedings Quantum Mind 2003: "Consciousness, Quantum Physics and the Brain", Convention Center and Leo Rich Theater, Tucson, Arizona.

111. Mershin, A., Nanopoulos D.V., Skoulakis E.M.C. (1999). Proceedings of the Academy of Athens, 74.

112. Mickey, B. \& Howard, J. (1995). Rigidity of microtubules is increased by stabilizing agents. J. Cell Biol., 130:909-917

113. Miller, J.P. (1980). Cytoplasmic resistivity of neurons in the lobster stomatogastric ganglion. http://cns.montana.edu/ zane/Cytoplasmic.htm 
114. Miller, J.P., Rall, W., \& Rinzel, J. (1985). Synaptic amplification by active membrane in dendritic spines. Brain Res., 325:325-330. http://cns.montana.edu/research/pub/synamp85/

115. Million, K., Larcher, J., Laoukili, J., Bourguignon, D., Marano, F. \& Tournier, F. (1999). Polyglutamylation and polyglycylation of $\alpha$ - and $\beta$-tubulins during in vitro ciliated cell differentiation of human respiratory epithelial cells. J. Cell Sci. 112, 4357-4366.

116. Miroshnichenko, A.E., Dmitriev, S.V., Vasiliev, A.A. \& Shigenari, T. (2000). Inelastic three-soliton collisions in a weakly discrete sine-Gordon system Nonlinearity 13: pp.837-848

117. Mitchison, T. \& Kirschner, M. (1984). Dynamic instability of microtubule growth. Nature. 312:237-242.

118. Mochon, C. (2003). Quantum computations using anyons from finite groups. http://www.theory.caltech.edu/ carlosm/publ/anyonAMStalk.pdf

119. Morse, P. M. \& Feshbach, H. (1953). "The Gradient." In Methods of Theoretical Physics, Part I. New York: McGraw-Hill, pp. 31-32.

120. Mukhopadhyay, K., Parrack, P. K. \& Bhattacharyya, B. (1990). The Carboxy Terminus of the $\alpha$-Subunit of Tubulin Regulates its Interaction with Colchicine. Biochemistry 29, 6845-6850.

121. Muller-Reichert, T., Chretien, D., Severin, F. \& Hyman, A.A. (1998). Structural changes at microtubule ends accompanying GTP hydrolysis: information from a slowly hydrolyzable analogue of GTP, guanylyl $(\alpha, \beta)$ methylenediphosphonate. Proc Natl Acad Sci U S A. 95:3661-3666.

122. Nave, C.R. (2003). HyperPhysics. http://hyperphysics.phyastr.gsu.edu/hbase/hph.html

123. Neuhaus, O., Archelos, J.J. \& Hartung, H.P. (2003). Immunomodulation in multiple sclerosis: from immunosuppression to neuroprotection. Trends in Pharmacological Sciences 24(3):131-138. 
124. Nieuwenhuys R. (1994). The neocortex. An overview of its evolutionary development, structural organization and synaptology. Anat Embryol (Berl) 190(4): 307-37.

125. Nogales, E., Wolf, S.G. \& Downing, K.H. (1998). Structure of the $\alpha / \beta$-tubulin dimer by electron crystallography. Nature 391 pp. 199

126. Overly, C.C., Rieff, H.I. \& Hollenbeck, P.J. (1996). Organelle motility and metabolism in axons vs. dendrites of cultured hippocampal neurons. Journal of Cell Science, 109, 971-80.

127. Penfield, W.G. (1954a). Some observations of the functional organization of the human brain. Proc. Am. phil. Soc. 98 (5), 293-297.

128. Penfield, W.G. (1954b). Studies of the cerebral cortex of man: a review and an interpretation. In: Brain mechanisms and consciousness (ed. J.F. Delafresnay), pp. 284-309. Oxford: Blackwell Scientific Publications.

129. Penfield, W.G. (1955). The role of the temporal cortex in certain psychical phenomena. J. ment. Sci. 101, 451-465.

130. Peters, A. \& Jones, E.G. (eds.) (1984). Cerebral Cortex. Volume 1: Cellular Components of the Cerebral Cortex. Plenum Press, NY.

131. Ponstingl, H., Krauhs, E., Little, M. \& Kempf, T. (1981). Complete amino acid sequence of $\alpha$-tubulin from porcine brain. Proc Natl Acad Sci USA 78:2757-2761.

132. Porter, M. (2003). Topological stabilization of quantum states in the microtubule. In Proceedings Quantum Mind 2003: "Consciousness, Quantum Physics and the Brain", Tucson, Arizona. http://www.geocities.com/Paris/Chateau/1500/power/topodraft1.ppt

133. Quarmby, L. (2000). Cellular Samurai: katanin and the severing of microtubules. Journal of Cell Science 113, 2821-2827. http://www.sfu.ca/biology/faculty/quarmby/main/lab/publications/samurai.p df 
134. Rai, S.S. \& Wolff, J. (1998). The $C$ terminus of $\beta$-tubulin regulates vinblastine-induced tubulin polymerization. Proc. Natl. Acad. Sci. USA 95:4253-4257

135. Rasmussen, S., Karampurwala, H., Vaidyanath, R., Jensen, K. \& Hameroff, S. (1990). Computational connectionism within neurons: a model of cytoskeletal automata subserving neural networks. Physica D 42:428-449.

136. Rodionov, V., Nadezhdina, E. \& Borisy, G. (1999). Centrosomal control of microtubule dynamics. Proc. Nat. Acad. Sci. USA 96, 115-120.

137. Rosenbaum, J. (2000). Cytoskeleton: functions for tubulin modifications at last. Curr. Biol. 10, R801-R803.

138. Sackett, D. L., Bhattacharyya, B. \& Wolff, J. (1985). Tubulin subunit carboxyl termini determine polymerization efficiency. J. Biol. Chem. 260, 43-45.

139. Sage, C.R., Dougherty, C.A., Davis, A.S., Burns, R.G., Wilson, L. \& Farrell, K.W. (1995). Site-directed mutagenesis of putative GTP-binding sites of yeast $\beta$-tubulin: evidence that $\alpha-, \beta$-, and $\gamma$-tubulins are atypical GTPases. Biochemistry. 34:16870.

140. Sajda, P. (2002). Computational Neural Modeling and Neuroengineering. BMEN E6480. Lecture 2: Cable Theory and Dendritic Trees. http://www.bme.columbia.edu/ sajda/bme6480/

141. Sarkar, T., Manna, T., Bhattacharyya, S., Mahapatra, P., Poddar, A., Roy, S., Pena, J., Solana, R., Tarazona, R. \& Bhattacharyya, B. (2001). Role of the Carboxy-Termini of Tubulin on Its Chaperone-Like Activity. Proteins: Structure, Function, and Genetics 44:262-269.

142. Sayer, R.J., Friedlander, M.J. and Redman, S.J. (1990). The time course and amplitude of EPSPs evoked at synapses between pairs of CA3/CA1 neurons in the hippocampal slice. Journal of Neuroscience Vol 10, 826-836. http://www.jneurosci.org/cgi/content/abstract/10/3/826 
143. Schey, H. M. (1997). Div, Grad, Curl, and All That: An Informal Text on Vector Calculus, 3rd ed. New York: W. W. Norton.

144. Schneider, A., Plessmann, U. \& Weber, K. (1997). Subpellicular and flagellar microtubules of Trypanosoma brucei are extensively glutamylated. J. Cell Sci. 110, 431-437.

145. Schneidman (2001). Noise and Information in Neural Codes. http://citeseer.nj.nec.com/595208.html

146. Schuessler, H.A., Kolomenskii, A.A., Mershin, A. \& Nanopoulos, D.V. (2003). Surface plasmon resonance study of the actin-myosin sarcomeric complex and tubulin dimers. http://www.arxiv.org/abs/quant-ph/0302159

147. Serrano, L., De La Torre, J., Maccioni, R. B. \& Avila, J. (1984). Involvement of the carboxyl-terminal domain of tubulin in the regulation of its assembly. Proc. Natl. Acad. Sci USA 81, 5989-5993.

148. Shepherd, G. \& Harris, K. (1998). Three-Dimensional Structure and Composition of CA3/CA1 Axons in Rat Hippocampal Slices: Implications for Presynaptic Connectivity and Compartmentalization. The Journal of Neuroscience, 18(20):8300-8310.

http://synapses.mcg.edu/pubs/Article2.pdf

149. Silflow, C. D. (1991). Why do tubulin gene families lack diversity in flagellate/ciliate protists? Protoplasma 164, 9-11.

150. Sirenko, Y.M., Stroscio, M.A. \& Kim, K.W. (1996). Elastic vibrations of microtubules in a fluid. Phys. Rev. E, 53:1003-1010

151. Smith, S., Watt, R.C. \& Hameroff, S.R. (1984). Cellular automata in cytoskeletal lattice proteins. Physica D 10:168-174.

152. Solomon, F. (1977). Binding sites for calcium on tubulin. Biochemistry 16(3):358-363.

153. Song, Y.-H. \& Mandelkow, E. (1993). Recombinant kinesin motor domain binds to beta-tubulin and decorates microtubules with a B surface lattice. $\begin{array}{llll}\text { Proc. Natl. Acad. Sci. USA 99, 1671-1675. } & \text { A }\end{array}$ http://www.pnas.org/cgi/reprint/90/5/1671.pdf 
154. Spruston N. (2000). Distant synapses raise their voices. Nature Neuroscience, 3:849-851.

155. Stewart, R.J., Farrell, K.W. \& Wilson, L. (1990). Role of GTP hydrolysis in microtubule polymerization: evidence for a coupled hydrolysis mechanism. Biochemistry. 29:6489-6498.

156. Stoilov, S., Rusanov, E., Mitev, D. \& Genkov, D. (1985). Biophysics. Publishing house "Medicine \& Physical Culture", Sofia.

157. Stracke, R., Boehm, K.J., Wollweber, L., Unger, E. \& Tuszynski, J. (2001). Transport and isopolar orientation of microtubules in electric fields. Experimental and theoretical implications. Submitted to Biophysics Journal. http://mitacs-gw.phys.ualberta.ca/mmpd/jackpaper3.pdf

158. Stracke, R., Boehm, K.J., Wollweber, L., Tuszynski, J.A., Unger E. (2002). Analysis of the migration behaviour of single microtubules in electric fields. Biochem. Biophys. Res. Commun. 293, 602-609.

159. Tran, P.T., Joshi, P. \& Salmon, E.D. (1997). How tubulin subunits are lost from the shortening ends of microtubules. J Struct Biol. 118:107-118.

160. Trpisova, B. \& Brown, J.A. (1998). Ordering of dipoles in different types of microtubule lattice. Int. J. Mod. Phys. B 12 543-578. http://citeseer.nj.nec.com/174728.html

161. Tuszynski, J. (2003). From tubulin to neurons: An overview of the physical properties of tubulin and their potential for affecting the functioning of neurons. In Proceedings Quantum Mind 2003: "Consciousness, Quantum Physics and the Brain", Convention Center and Leo Rich Theater, Tucson, Arizona.

162. Tuszynski, J., Hameroff, S., Sataric, M.V., Trpisova, B. \& Nip, M.L.A. (1995). Ferroelectric behavior in microtubule dipole lattices; implications for information processing, signaling and assembly/disassembly. J. Theor. Biol. 174:371-380 
163. Tuszynski, J.A., Trpisova, B., Sept, D. \& Brown, J.A. (1997a). Physical Issues in the Structure and Function of Microtubules. Journal Of Structural Biology 118 94-106. http://citeseer.nj.nec.com/351883.html

164. Tuszynski, J.A., Sept, D. \& Brown, J.A. (1997b). Polymerization, energy transfer and dielectric polarization of microtubules. La Physique au Canada, 237-246. http://citeseer.nj.nec.com/356882.html

165. Tuszynski, J.A., Brown, J.A. \& Hawrylak, P. (1998). Dielectric Polarization, Electrical Conduction, Information Processing and Quantum Computation in Microtubules, are they Plausible? Phil. Trans. A. Proc. Roy. Soc. (London) 356:1897-1926. http://citeseer.nj.nec.com/364820.html

166. Ulfhake, B. \& Kellerth, J.O. (1981). A quantitative light microscopic study of the dendrites of cat spinal $\alpha$-motoneurons after intracellular staining with horseradish peroxidase. Journal of Comparative Neurology, 202, 571583.

167. Uppuluri, S., Knipling, L., Sackett, D. L. \& Wolff, J. (1993). Localization of the Colchicine-Binding Site of Tubulin. Proc. Natl. Acad. Sci. USA 90, 11598-11602.

168. Vassilev, P.M., Dronzine, R.T., Vassileva, M.P. \& Georgiev, G.A. (1982). Parallel arrays of microtubules formed in electric and magnetic fields. Bioscience Rep. 2:1025-1029.

169. Vater, W., Stracke, R., Boehm, K.J., Speicher, C., Weber, P. \& Unger, E. (1998). Behaviour of individual microtubules and microtubule bundles in electric fields. Sixth Foresight Conference on Molecular Nanotechnology.

170. Voter, W.A., O'Brien, E.T. \& Erickson, H.P. (1991). Dilution-induced disassembly of microtubules: relation to dynamic instability and the GTP cap. Cell Motil Cytoskeleton. 18:55-62.

171. Wadati, M. (2001). Introduction to solitons. Pramana - Journal of Physics Vol. 57 (5 -6) pp. 841-847 
172. Waldrop, B. \& Glantz, R.M. (1985). Synaptic mechanisms of a tonic EPSP in crustacean visual interneurons: analysis and simulation. Journal of Neurophysiology, Vol 54, Issue 3: 636-650.

173. Walker, R.A., Pryer, N.K. \& Salmon, E.D. (1991). Dilution of individual microtubules observed in real time in vitro: evidence that cap size is small and independent of elongation rate. J Cell Biol. 114:73-81.

174. Weisenberg, R.C., Deery, W.J. \& Dickinson, P.J. (1976). Tubulinnucleotide interactions during the polymerization and depolymerization of microtubules. Biochemistry. 15:4248-4254.

175. Weisstein, E.W. (2003). Gradient. Wolfram Research, Inc.

176. Wikswo, J.P., Barach, J.P. \& Freeman, J.A. (1980). Magnetic Field of a Nerve Impulse: First Measurements. Science, Vol 208 (4439), pp 53-55. http://www.vanderbilt.edu/lsp/abstracts/wikswo-s-1980.htm

177. Zettlmeissl, G., Rudolph, R. \& Jaenicke, R. (1979). Reconstitution of lactic dehydrogenase. Noncovalent aggregation vs. reactivation. 1. Physical properties and kinetics of aggregation. Biochemistry 18: 5567-5571.

178. Zlatev, M.P. (1972). Theoretical Electrotechnics. Volume I. Publishing house "Technics", Sofia. 